\title{
INTRODUCTION TO BOUNDARY VALUE PROBLEMS OF NONLINEAR ELASTOSTATICS
}

Dedicated to the memory of Professor Mutsuhide Matsumura (1931-2006)

By

Kazuaki TAIRA

\begin{abstract}
This paper provides a careful and accessible exposition of an $L^{p}$ approach to boundary value problems of nonlinear elastostatics in the case where solutions of the linearized problem correspond faithfully to those of the nonlinear problem, that is, in the case where there is no bifurcation. We prove that if the linearized problem has unique solutions, then so does the nonlinear one, nearby. This is done by using the linear $L^{p}$ theory and the inverse mapping theorem. The main theorem can be applied to the Saint Venant-Kirchhoff elastic material and the Hencky-Nadai elastoplastic material in a unified theory. The approach here is distinguished by the extensive use of the ideas and techniques characteristic of the recent developments in the theory of partial differential equations.
\end{abstract}

\section{Table of Contents}

1. Introduction and Main Results

1.1 Formulation of a Problem

1.2 Statement of Main Results

2. Theory of Pseudo-Differential Operators

2.1 Function Spaces

2.2 Pseudo-Differential Operators

3. Linear Elastostatics

3.1 Linearization of Nonlinear Elastostatics

3.2 Existence and Uniqueness Theorem for Problem (1.4)

2000 Mathematics Subject Classification. Primary 73C50, 73C35; Secondary 35J65.

Key words and phrases. nonlinear elastostatics, displacement-traction boundary condition, Saint Venant-Kirchhoff elastic material, Hencky-Nadai elasto-plastic material.

Received May 7, 2007. 
4. Reduction to the Boundary

4.1 Operator $\boldsymbol{T}_{\alpha}$

4.2 Operator $\boldsymbol{\Pi}$

5. Regularity Theorem for Problem (1.4)

6. Uniqueness Theorem for Problem (1.4)

7. Existence Theorem for Problem (1.4)

7.1 Proof of Theorem 7.1

7.2 Proof of Proposition 7.4

8. Proof of Theorems

8.1 Proof of Main Theorem

8.2 Proof of Theorem 1.1

8.3 Proof of Theorem 1.2

9. Summary and Discussion

References

\section{Introduction and Main Results}

This paper is devoted to an $L^{p}$ approach to boundary value problems of nonlinear elastostatics in the case where solutions of the linearized problem correspond faithfully to those of the nonlinear problem. We prove that if the linearized problem has unique solutions, then so does the nonlinear one, nearby (Main Theorem). This is done by using the $L^{p}$ theory of pseudo-differential operators and the inverse mapping theorem. Our boundary condition is a "regularization" of the genuine mixed displacement-traction boundary condition; more precisely, it is a smooth linear combination of displacement and traction boundary conditions, but is not equal to the pure traction boundary condition. Moreover, it should be emphasized that our problem becomes a degenerate elliptic boundary value problem from an analytical point of view. The crucial point is how to find a function space associated with the degenerate boundary condition in which the linearized problem has unique solutions. Main Theorem can be applied to the Saint Venant-Kirchhoff elastic material and the HenckyNadai elasto-plastic material in a unified theory (Theorem 1.1 and Theorem 1.2). The approach here is distinguished by the extensive use of the ideas and techniques characteristic of the recent developments in the theory of partial differential equations ([Ta4]). This expository paper is an expanded and revised version of the previous paper [Ta3]. 


\subsection{Formulation of a Problem}

We start with the basic equations for continuum mechanics, providing a quick survey of a few standard topic in elasticity theory from a classical point of view. The treatment is exclusively in Euclidean space $\mathbf{R}^{3}$, by using standard Euclidean coordinates. For more thorough treatments of this subject, the reader might be referred to Ciarlet [Ci] and Marsden-Hughes [MH].

Let $\Omega$ be an open, connected subset of $\mathbf{R}^{3}$ with piecewise smooth boundary $\partial \Omega$. We think of its closure $\bar{\Omega}=\Omega \cup \partial \Omega$ as representing the volume occupied by an undeformed body; so the set $\mathscr{B}=\bar{\Omega}$ is called the reference configuration. A deformation of the reference configuration $\mathscr{B}$ is a vector field

$$
\boldsymbol{\phi}=\left(\begin{array}{c}
\phi_{1} \\
\phi_{2} \\
\phi_{3}
\end{array}\right): \mathscr{B} \rightarrow \mathbf{R}^{3}
$$

which is a continuously differentiable, orientation-preserving and invertible map. Given a reference configuration $\mathscr{B}$ and a deformation $\phi: \mathscr{B} \rightarrow \mathbf{R}^{3}$, the set $\phi(\mathscr{B})$ is called the deformed configuration (see Figure 1.1). Points in $\mathscr{B}$ are denoted by $X=\left(X_{1}, X_{2}, X_{3}\right) \in \mathscr{B}$ and are called material points, while points in $\mathbf{R}^{3}$ are denoted by $x=\left(x_{1}, x_{2}, x_{3}\right) \in \mathbf{R}^{3}$ and are called spatial points. We write as

$$
x=\phi(X) ; \quad x_{i}=\phi_{i}(X) .
$$

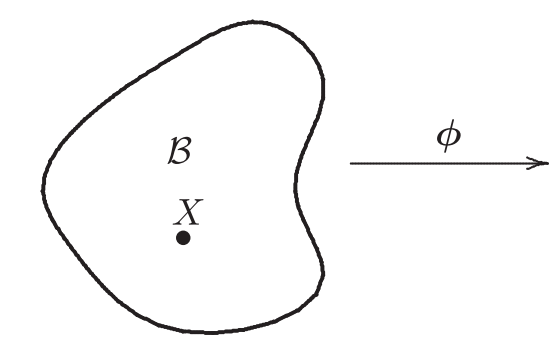

Reference configuration

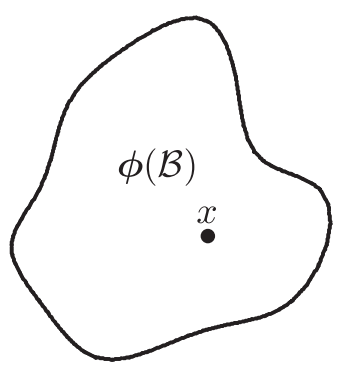

Body

Figure 1.1

The $3 \times 3$ matrix of partial derivatives of $\boldsymbol{\phi}$ is denoted by $\boldsymbol{F}(X)=\boldsymbol{\nabla} \boldsymbol{\phi}(X)$, and is called the deformation gradient: 


$$
\boldsymbol{F}(X)=\left(\begin{array}{lll}
F_{11} & F_{12} & F_{13} \\
F_{21} & F_{22} & F_{23} \\
F_{31} & F_{32} & F_{33}
\end{array}\right), \quad F_{i j}=\frac{\partial \phi_{i}}{\partial X_{j}} .
$$

The deformation gradient is simply the matrix representing the Fréchet derivative of the mapping $\boldsymbol{\phi}$. We remark that $\operatorname{det} \boldsymbol{F}>0$, since the mapping $\boldsymbol{\phi}$ is orientationpreserving.

It is often convenient to introduce the displacement $\boldsymbol{u}$ of $\mathscr{B}$, which is a vector field

$$
\boldsymbol{u}=\left(\begin{array}{c}
u_{1} \\
u_{2} \\
u_{3}
\end{array}\right): \mathscr{B} \rightarrow \mathbf{R}^{3}
$$

defined by the formula

$$
\boldsymbol{u}:=\phi-\text { id },
$$

where id is the identity map of $\mathbf{R}^{3}$ onto itself. Componentwise, the displacement $\boldsymbol{u}$ of $\mathscr{B}$ can be written as follows:

$$
\boldsymbol{u}(X)=\left(\begin{array}{l}
u_{1}(X) \\
u_{2}(X) \\
u_{3}(X)
\end{array}\right):=\left(\begin{array}{l}
\phi_{1}(X)-X_{1} \\
\phi_{2}(X)-X_{2} \\
\phi_{3}(X)-X_{3}
\end{array}\right) .
$$

The $3 \times 3$ matrix of partial derivatives of $\boldsymbol{u}$ is denoted by $\boldsymbol{\nabla} \boldsymbol{u}$, and is called the displacement gradient. We remark that

$$
\nabla \boldsymbol{u}=\left(\begin{array}{lll}
\frac{\partial u_{1}}{\partial X_{1}} & \frac{\partial u_{1}}{\partial X_{2}} & \frac{\partial u_{1}}{\partial X_{3}} \\
\frac{\partial u_{2}}{\partial X_{1}} & \frac{\partial u_{2}}{\partial X_{2}} & \frac{\partial u_{2}}{\partial X_{3}} \\
\frac{\partial u_{3}}{\partial X_{1}} & \frac{\partial u_{3}}{\partial X_{2}} & \frac{\partial u_{3}}{\partial X_{3}}
\end{array}\right),
$$

so that

$$
\nabla \boldsymbol{u}=\nabla \phi-I
$$

where $\boldsymbol{I}=\left(\delta_{i j}\right)$ is the $3 \times 3$ unit matrix.

The symmetric two-tensor

$$
\boldsymbol{C}:={ }^{t} \boldsymbol{F} \boldsymbol{F} ; \quad C_{i j}:=\sum_{k=1}^{3} F_{k i} F_{k j},
$$

is called the (right) Cauchy-Green strain tensor. 


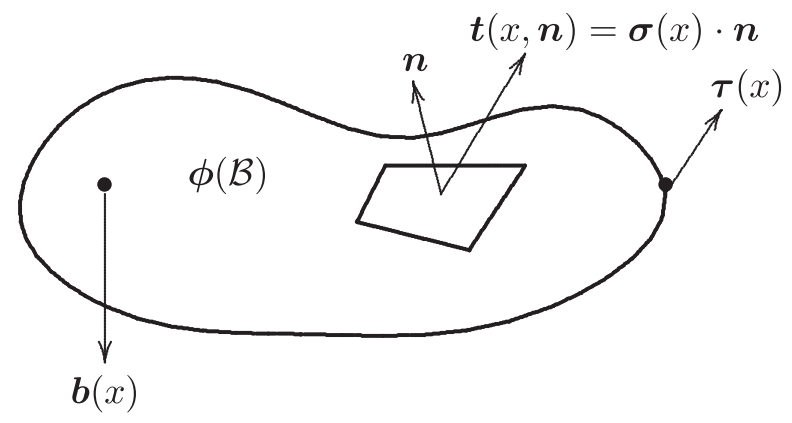

Figure 1.2

A body $\boldsymbol{\phi}(\mathscr{B})$ is acted on by applied body forces $\boldsymbol{b}(x)$ in its interior and by applied surface forces $\boldsymbol{\tau}(x)$ on a portion of the boundary. The pair $(\boldsymbol{b}, \boldsymbol{\tau})$ of forces is called the load, and is given or prescribed in advance (see Figure 1.2). An applied body force is called a dead load if its associated density per unit volume in the reference configuration is independent of the particular deformation $\phi$ considered. This is the case of the gravity field. Similarly, an applied surface force is called a dead load if its associated density per unit area in the reference configuration is independent of the particular deformation $\phi$ considered. This is the case where a portion of the boundary of the body is held fixed, while the remaining portion is considered as free from all external actions (see Figure 1.3).

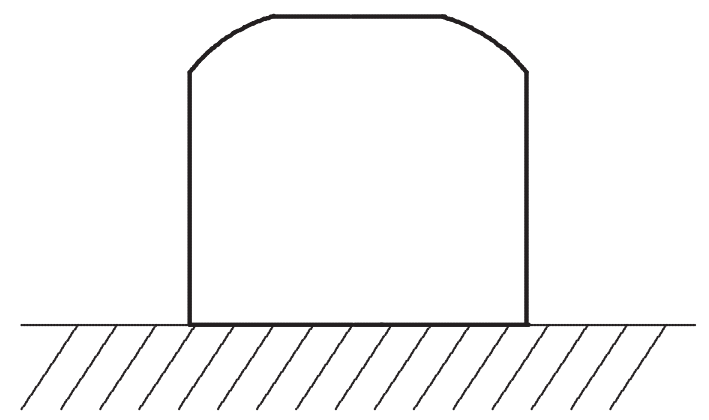

Figure 1.3

In addition, the body generally experiences internal forces of stress across any given surface. Let $\boldsymbol{t}(x, \boldsymbol{n})$ be the force at position $x$ across an oriented surface element with unit outward normal $\boldsymbol{n}$ (see Figure 1.2). We present the basic three equations for continuum mechanics in nonlinear elastostatics. 
(1) First, the celebrated Cauchy theorem asserts (cf. [Ci, Chapter 2]; [MH, Chapter 2]) that if the balance of momentum holds true, then the stress vector $\boldsymbol{t}(x, \boldsymbol{n})=\left(t_{i}(x, \boldsymbol{n})\right)$ depends linearly on $\boldsymbol{n}$, that is, there exists a two-tensor $\boldsymbol{\sigma}(x)=$ $\left(\sigma_{i j}(x)\right)$ such that

$$
\boldsymbol{t}(x, \boldsymbol{n})=\boldsymbol{\sigma}(x) \cdot \boldsymbol{n} ; \quad t_{i}(x, \boldsymbol{n})=\sum_{j=1}^{3} \sigma_{i j}(x) n_{j} .
$$

The vector $\boldsymbol{t}(x, \boldsymbol{n})$ is called the Cauchy stress vector and the tensor $\boldsymbol{\sigma}(x)$ is called the Cauchy stress tensor.

(2) Secondly, the balance of angular momentum leads to the symmetry of the two-tensor $\sigma(x): \sigma_{i j}(x)=\sigma_{j i}(x)$.

(3) Thirdly, the balance of linear momentum leads to the nonlinear equations of elastostatics: $\operatorname{div} \boldsymbol{\sigma}+\rho(x) \boldsymbol{b}(x)=0$ where $\rho(x)$ is the mass density in the deformed configuration $\phi(\mathscr{B})$.

The vector $\boldsymbol{T}(X, \boldsymbol{N})$, defined by the formula

$$
\begin{aligned}
& \boldsymbol{T}(X, \boldsymbol{N}):=\boldsymbol{P}(X) \cdot \boldsymbol{N} \\
& \boldsymbol{P}(X):=\operatorname{det}(\boldsymbol{\nabla} \boldsymbol{\phi}(X)) \boldsymbol{\sigma}(\boldsymbol{\phi}(X)) \cdot\left({ }^{t} \boldsymbol{\nabla} \boldsymbol{\phi}(X)\right)^{-1},
\end{aligned}
$$

is called the first Piola-Kirchhoff stress vector, where $\boldsymbol{N}$ is the unit outward normal at $X$. It should be emphasized that the vector $\boldsymbol{T}(X, \boldsymbol{N})$ is parallel to the Cauchy stress vector $\boldsymbol{t}(x, \boldsymbol{n})$, but measures the force per unit undeformed area with normal $\boldsymbol{N}$ (see Figure 1.4). The two-tensor $\boldsymbol{P}(X)=\left(P_{i j}(X)\right)$, which is the Piola transform of the Cauchy stress tensor $\boldsymbol{\sigma}(x)$, is called the first PiolaKirchhoff stress tensor.
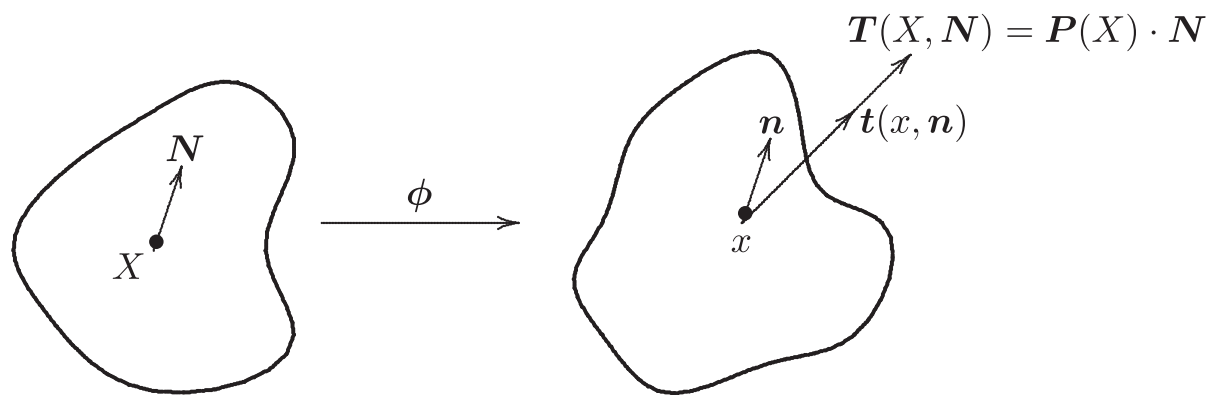

Figure 1.4 
The second Piola-Kirchhoff stress vector is obtained by transforming $\boldsymbol{T}=$ $\boldsymbol{P} \cdot \boldsymbol{N}$, which is a vector at $x$, to a vector based at $X$ by setting $\mathfrak{I}=\boldsymbol{F}^{-1} \cdot \boldsymbol{T}$. Hence $\mathfrak{I}=\boldsymbol{S} \cdot \boldsymbol{N}$, where $\boldsymbol{S}=\boldsymbol{F}^{-1} \boldsymbol{P}$. Componentwise, this can be written as follows:

$$
S_{i j}(X)=\sum_{k=1}^{3} \frac{\partial X_{i}}{\partial x_{k}} P_{k j}(X) .
$$

The $3 \times 3$ matrix $\boldsymbol{S}(X)=\left(S_{i j}(X)\right)$ is called the second Piola-Kirchhoff stress tensor.

The following three diagrams give a bird's eye view of basic equations in nonlinear elastostatics from a classical point of view:

\begin{tabular}{|c|c|}
\hline Cauchy's theorem & Balance of momentum \\
\hline Eulerian form & $\boldsymbol{t}(x, \boldsymbol{n})=\boldsymbol{\sigma}(x) \cdot \boldsymbol{n}$ \\
\hline Lagrangian form & $\boldsymbol{T}(X, \boldsymbol{N})=\boldsymbol{P}(X) \cdot \boldsymbol{N}$ \\
\hline
\end{tabular}

\begin{tabular}{|c|c|}
\hline & Balance of angular momentum \\
\hline Eulerian form & $\boldsymbol{\sigma}(x)={ }^{t} \boldsymbol{\sigma}(x)$ \\
\hline Lagrangian form & $\boldsymbol{S}(X)={ }^{t} \boldsymbol{S}(X)$ \\
\hline
\end{tabular}

\begin{tabular}{|c|c|}
\hline & Balance of linear momentum \\
\hline Eulerian form & $\operatorname{div} \boldsymbol{\sigma}+\rho(x) \boldsymbol{b}(x)=0$ \\
\hline Lagrangian form & $\begin{array}{c}\operatorname{DIV} \boldsymbol{P}+\rho_{0}(X) \boldsymbol{B}(X)=0 \\
\rho_{0}(X)=\rho(\boldsymbol{\phi}(X)) \operatorname{det}(\boldsymbol{\nabla} \boldsymbol{\phi}(X))\end{array}$ \\
\hline
\end{tabular}

A material is said to be elastic if we can write the first Piola-Kirchhoff stress tensor $\boldsymbol{P}(X)=\left(P_{i j}(X)\right)$ as a function $\hat{\boldsymbol{P}}(X, \boldsymbol{F})=\left(\hat{\boldsymbol{P}}_{i j}(X, \boldsymbol{F})\right)$ of points $X \in \mathscr{B}$ and $3 \times 3$ matrices $\boldsymbol{F}$ with $\operatorname{det} \boldsymbol{F}>0$ such that

$$
\boldsymbol{P}(X)=\hat{\boldsymbol{P}}(X, \nabla \phi(X))
$$

Specification of a function $\hat{\boldsymbol{P}}(X, \boldsymbol{F})$ for an elastic material is called a stress-strain law, and the function $\hat{\boldsymbol{P}}(X, \boldsymbol{F})$ is called a constitutive function.

An elastic material is said to be hyperelastic if there exists a smooth function $W(X, \boldsymbol{F})$ of points $X \in \mathscr{B}$ and $3 \times 3$ matrices $\boldsymbol{F}$ with $\operatorname{det} \boldsymbol{F}>0$ such that 


$$
\hat{\boldsymbol{P}}(X, \boldsymbol{F})=\frac{\partial W}{\partial \boldsymbol{F}}(X, \boldsymbol{F}) ; \quad \hat{\boldsymbol{P}}_{i j}(X, \boldsymbol{F})=\frac{\partial W}{\partial F_{i j}}(X, \boldsymbol{F}) .
$$

The function $W(X, \boldsymbol{F})$ is called a stored energy function. The four-index tensor $\mathbf{A}(X, \boldsymbol{F})=(\partial \hat{\boldsymbol{P}} / \partial \boldsymbol{F})(X, \boldsymbol{F})=\left(\partial^{2} W / \partial \boldsymbol{F} \partial \boldsymbol{F}\right)(X, \boldsymbol{F})$, defined by the formula

$$
\mathrm{A}_{i j \ell m}(X, \boldsymbol{F}):=\frac{\partial \hat{\boldsymbol{P}}_{i j}}{\partial F_{\ell m}}(X, \boldsymbol{F})=\frac{\partial^{2} W}{\partial F_{i j} \partial F_{\ell m}}(X, \boldsymbol{F}),
$$

is called the first elasticity tensor.

Some boundary conditions often encountered are the following:

(a) The boundary condition of place: $\phi(X)=\phi_{d}(X)$ is described for $X \in \partial \mathscr{B}$.

(b) The boundary condition of traction: $\hat{\boldsymbol{P}}(X, \nabla \boldsymbol{\phi}(X)) \cdot \boldsymbol{N}(X)=\boldsymbol{\tau}(X)$ is described for $X \in \partial \mathscr{B}$, where $\boldsymbol{N}(X)$ is the unit outward normal to $\partial \mathscr{B}$ at $X$.

We make the following two assumptions (H.1) and (H.2) throughout the paper:

(H.1) The reference configuration is a bounded region $\mathscr{B}=\bar{\Omega} \subset \mathbf{R}^{3}$ with smooth boundary $\partial \Omega$.

(H.2) The material is hyperelastic.

We give two important examples of stored energy functions for hyperelastic materials:

EXAmPle 1.1 (The Hencky-Nadai elasto-plastic material). The stored energy function $W(X, \boldsymbol{F})$ has the form

$$
W(X, \boldsymbol{F})=\frac{3}{4} \int_{0}^{\Gamma(\boldsymbol{F})} g(\xi) d \xi+\frac{K}{2}\left(\sum_{k=1}^{3} F_{k k}-3\right)^{2}
$$

where $g \in C^{\infty}([0, \infty), \mathbf{R})$, the constant $K$ is the modulus of compression and

$$
\Gamma(\boldsymbol{F})=\frac{4}{3} \sum_{i, j=1}^{3}\left(\frac{1}{2}\left(F_{i j}+F_{j i}\right)-\frac{1}{3}\left(\sum_{k=1}^{3} F_{k k} F_{k k}\right) \delta_{i j}\right)^{2}
$$

The first Piola-Kirchhoff stress tensor $\boldsymbol{P}(X)=\left(P_{i j}(X)\right)$ is given by the formula 


$$
\begin{aligned}
P_{i j}(X)= & \hat{\boldsymbol{P}}_{i j}(X, \boldsymbol{F}(X)) \\
= & \frac{\partial W}{\partial F_{i j}}(X, \boldsymbol{F}(X)) \\
= & \left(K-\frac{2}{3} g(\Gamma(\boldsymbol{F}(X)))\left(\sum_{k=1}^{3} F_{k k}(X)-3\right) \delta_{i j}\right. \\
& +g(\Gamma(\boldsymbol{F})(X))\left(F_{i j}(X)+F_{j i}(X)-2 \delta_{i j}\right),
\end{aligned}
$$

where

$$
\boldsymbol{F}(X)=\left(F_{i j}(X)\right)=\left(\frac{\partial \phi_{i}}{\partial X_{j}}\right)
$$

EXAmple 1.2 (The Saint Venant-Kirchhoff isotropic material). The stored energy function $W(X, \boldsymbol{F})$ has the form

$$
W(X, \boldsymbol{F})=\frac{\lambda(X)}{8}\left(\sum_{k=1}^{3} C_{k k}(\boldsymbol{F})-3\right)^{2}+\frac{\mu(X)}{4} \sum_{i, j=1}^{3}\left(C_{i j}(\boldsymbol{F})-\delta_{i j}\right)^{2},
$$

where $\lambda(X), \mu(X)$ are smooth Lamé functions and the two-tensor

$$
C_{i j}(\boldsymbol{F})=\sum_{k=1}^{3} F_{k i} F_{k j}
$$

is the (right) Cauchy-Green strain tensor.

The first Piola-Kirchhoff stress tensor $\boldsymbol{P}(X)=\left(P_{i j}(X)\right)$ is given by the formula

$$
\begin{aligned}
P_{i j}(X)= & \hat{P}_{i j}(X, \boldsymbol{F}(X)) \\
= & \frac{\partial W}{\partial F_{i j}}(X, \boldsymbol{F}(X)) \\
= & {\left[\frac{\lambda(X)}{2}\left(\sum_{k=1}^{3} C_{k k}(\boldsymbol{F}(X))-3\right)-\mu(X)\right] F_{i j}(X) } \\
& +\mu(X) \sum_{m=1}^{3} F_{i m}(X) C_{m j}(\boldsymbol{F}(X)),
\end{aligned}
$$

where

$$
\boldsymbol{F}(X)=\left(F_{i j}(X)\right)=\left(\frac{\partial \phi_{i}}{\partial X_{j}}\right) .
$$


REMARK 1.1. If we define the Green-Saint Venant strain tensor $\boldsymbol{E}=\left(E_{i j}\right)$ by the formula

$$
\boldsymbol{E}:=\frac{1}{2}(\boldsymbol{C}-\boldsymbol{I})
$$

or componentwise,

$$
E_{i j}:=\frac{1}{2}\left(\frac{\partial u_{i}}{\partial X_{j}}+\frac{\partial u_{j}}{\partial X_{i}}+\sum_{k=1}^{3} \frac{\partial u_{k}}{\partial X_{i}} \frac{\partial u_{k}}{\partial X_{j}}\right)
$$

then it is easy to see that the stored energy function $W(X, \boldsymbol{F})$ in Example 1.2 can be written in the form (cf. [MH, Chapter 4, Proposition 3.12])

$$
W(X, \boldsymbol{F})=\frac{\lambda(X)}{2}(\operatorname{tr} \boldsymbol{E})^{2}+\mu(X) \operatorname{tr}\left(\boldsymbol{E}^{2}\right) .
$$

It should be noticed that the first Piola-Kirchhoff stress tensors in Examples 1.1 and 1.2 are not linear functions of the deformation gradient $\boldsymbol{F}$, which leads to equations of nonlinear elastostatics.

Let $\boldsymbol{B}: \Omega \rightarrow \mathbf{R}^{3}$ be the density of a given body force per unit volume in the reference configuration and $\tau: \partial \Omega \rightarrow \mathbf{R}^{3}$ the density of a given surface force per unit area in the reference configuration (see Figure 1.5).

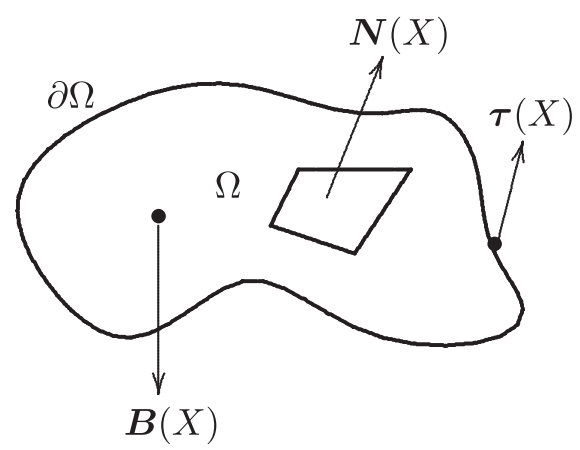

Figure 1.5

We consider the following equilibrium equations for the unknown deformation $\phi$ :

$$
\begin{cases}\operatorname{DIV} \hat{\boldsymbol{P}}(X, \nabla \phi(X))+\boldsymbol{B}(X)=\mathbf{0} & \text { in } \Omega \\ \alpha(X) \hat{\boldsymbol{P}}(X, \nabla \boldsymbol{\phi}(X)) \cdot \boldsymbol{N}(X)+(1-\alpha(X)) \boldsymbol{\phi}(X)=\boldsymbol{\tau}(X) & \text { on } \partial \Omega\end{cases}
$$


Here $\alpha(X)$ is a smooth function on $\partial \Omega$ such that

$$
0 \leq \alpha(X) \leq 1 \quad \text { on } \partial \Omega
$$

We remark that our boundary condition is a "regularization" of the genuine mixed displacement-traction boundary condition; more precisely, it is a smooth linear combination of displacement and traction boundary conditions, but is not equal to the pure traction boundary condition (see Figure 1.6). Moreover, it should be emphasized that our problem (1.1) becomes a degenerate elliptic boundary value problem from an analytical point of view. This is due to the fact that the so-called Shapiro-Lopatinskii complementary condition is violated at the points $X \in \partial \Omega$ where $\alpha(X)=0$. Marsden-Hughes $[\mathrm{MH}]$ studied the nondegenerate case. More precisely, they assume that the boundary $\partial \Omega$ is the disjoint union of the two closed subsets $\Gamma_{0}=\{X \in \partial \Omega: \alpha(X)=0\}$ and $\partial \Omega \backslash \Gamma_{0}=$ $\{X \in \partial \Omega: \alpha(X)>0\}$.

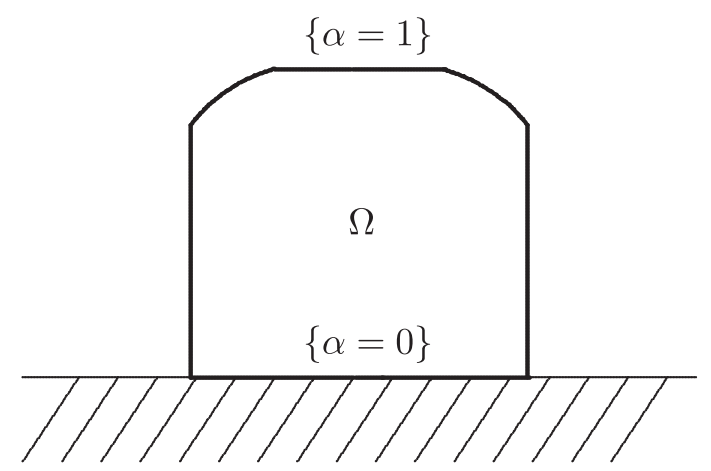

Figure 1.6

\subsection{Statement of Main Results}

We study the nonlinear problem (1.1) in the framework of Sobolev spaces of $L^{p}$ type. The process of linearization provides a key link between the linear and nonlinear theories of elasticity. The crucial point is how to find a function space associated with the boundary condition (1.2) in which the linearized problem has unique solutions (see [Ta2]).

If $s \in \mathbf{R}$ and $1<p<\infty$, we define the Sobolev space (see Section 2.1)

$$
H^{s, p}(\Omega)=\text { the space of restrictions to } \Omega \text { of functions in } H^{s, p}\left(\mathbf{R}^{n}\right) \text {. }
$$


The space $H^{s, p}(\Omega)$ is a Banach space with the norm

$$
\|u\|_{s, p}=\inf \left\{\|U\|_{s, p}: U \in H^{s, p}\left(\mathbf{R}^{n}\right),\left.U\right|_{\Omega}=u\right\},
$$

where the infimum is taken over all functions $U \in H^{s, p}\left(\mathbf{R}^{n}\right)$ which equal $u$ in $\Omega$.

Furthermore, if $s>1 / p$, we define the boundary space

$B^{s-1 / p, p}(\partial \Omega)=$ the space of the boundary values of functions in $H^{s, p}(\Omega)$.

In the space $B^{s-1 / p, p}(\partial \Omega)$, we introduce a norm

$$
|\varphi|_{s-1 / p, p}=\inf \left\{\|u\|_{s, p}: u \in H^{s, p}(\Omega),\left.u\right|_{\partial \Omega}=\varphi\right\},
$$

where the infimum is taken over all functions $u \in H^{s, p}(\Omega)$ which equal $\varphi$ on $\partial \Omega$. The space $B^{s-1 / p, p}(\partial \Omega)$ is a Banach space with respect to this norm $|\cdot|_{s-1 / p, p}$; more precisely, it is a Besov space (cf. $[\mathrm{AF}],[\mathrm{BL}],[\mathrm{Tr}]$ ).

Now we let

$$
\begin{aligned}
& \boldsymbol{H}^{s, p}\left(\Omega, \mathbf{R}^{3}\right):=\text { the space of all } H^{s, p} \text { functions } \phi: \Omega \rightarrow \mathbf{R}^{3}, \\
& \boldsymbol{B}^{s-1 / p, p}\left(\partial \Omega, \mathbf{R}^{3}\right):=\text { the space of all } B^{s-1 / p, p} \text { functions } \boldsymbol{\varphi}: \partial \Omega \rightarrow \mathbf{R}^{3} .
\end{aligned}
$$

We introduce a subspace of the Besov space $\boldsymbol{B}^{s-1-1 / p, p}\left(\partial \Omega, \mathbf{R}^{3}\right)$ which is associated with the boundary condition

$$
\left\{\begin{array}{l}
\alpha(X) \hat{\boldsymbol{P}}(\boldsymbol{\nabla} \boldsymbol{\phi}) \cdot \boldsymbol{N}+(1-\alpha(X)) \boldsymbol{\phi}=\boldsymbol{\tau} \quad \text { on } \partial \boldsymbol{\Omega} \\
0 \leq \alpha(X) \leq 1 \quad \text { on } \partial \Omega
\end{array}\right.
$$

as follows: We let

$$
\begin{gathered}
\boldsymbol{B}_{(\alpha)}^{s-1-1 / p, p}\left(\partial \Omega, \mathbf{R}^{3}\right)=\left\{\boldsymbol{\phi}=\alpha(X) \boldsymbol{\phi}_{1}+(1-\alpha(X)) \boldsymbol{\phi}_{2}: \boldsymbol{\phi}_{1} \in \boldsymbol{B}^{s-1-1 / p, p}\left(\partial \Omega, \mathbf{R}^{3}\right),\right. \\
\left.\boldsymbol{\phi}_{2} \in \boldsymbol{B}^{s-1 / p, p}\left(\partial \Omega, \mathbf{R}^{3}\right)\right\},
\end{gathered}
$$

and define the norm

$$
|\boldsymbol{\phi}|_{\alpha ; s-1-1 / p, p}=\inf \left\{\left|\phi_{1}\right|_{s-1-1 / p, p}+\left|\phi_{2}\right|_{s-1 / p, p}: \boldsymbol{\phi}=\alpha(X) \boldsymbol{\phi}_{1}+(1-\alpha(X)) \boldsymbol{\phi}_{2}\right\} .
$$

Then it is easy to verify (see [Ta2]) that the space $\boldsymbol{B}_{(\alpha)}^{s-1-1 / p, p}\left(\partial \Omega, \mathbf{R}^{3}\right)$ is a Banach space with the norm $|\cdot|_{\alpha ; s-1-1 / p, p}$. It is worth while pointing out here that

$$
\begin{aligned}
& \boldsymbol{B}_{(\alpha)}^{s-1-1 / p, p}\left(\partial \Omega, \mathbf{R}^{3}\right) \\
& \quad= \begin{cases}\boldsymbol{B}^{s-1 / p, p}\left(\partial \Omega, \mathbf{R}^{3}\right) & \text { if } \alpha(X) \equiv 0 \text { on } \partial \Omega \text { (the pure displacement case) } \\
\boldsymbol{B}^{s-1-1 / p, p}\left(\partial \Omega, \mathbf{R}^{3}\right) & \text { if } \alpha(X) \equiv 1 \text { on } \partial \Omega \text { (the pure traction case) } .\end{cases}
\end{aligned}
$$


If $s>3 / p+1$, we let

$$
\mathscr{X}=\text { the space } \boldsymbol{H}^{s, p}\left(\Omega, \mathbf{R}^{3}\right) \text { of all } H^{s, p} \text { vector functions } \boldsymbol{\phi},
$$

and

$$
\mathscr{C}=\text { the subspace of all configurations } \phi \text { in } \mathscr{X} \text {. }
$$

It should be emphasized that the set $\mathscr{C}$ is open in the space $\mathscr{X}$. Indeed, this follows from an application of the inverse mapping theorem, since the Sobolev imbedding theorem asserts that the $H^{s, p}$ topology is stronger than the $C^{1}$ topology for $s>3 / p+1$ (see [AF, Theorem 4.12]). We let

$$
\mathscr{Y}=\text { the space of all pairs }(\boldsymbol{B}, \boldsymbol{\tau}) \in \boldsymbol{H}^{s-2, p}\left(\boldsymbol{\Omega}, \mathbf{R}^{3}\right) \times \boldsymbol{B}_{(\alpha)}^{s-1-1 / p, p}\left(\partial \Omega, \mathbf{R}^{3}\right) .
$$

We associate with problem (1.1) a nonlinear mapping between Banach spaces

$$
F: \mathscr{X} \rightarrow \mathscr{Y}
$$

as follows:

$$
F(\boldsymbol{\phi})=\left(-\operatorname{DIV} \hat{\boldsymbol{P}}(\nabla \boldsymbol{\phi}), \alpha(X) \hat{\boldsymbol{P}}(\nabla \boldsymbol{\phi}) \cdot \boldsymbol{N}+\left.(1-\alpha(X)) \boldsymbol{\phi}\right|_{\partial \Omega}\right), \quad \boldsymbol{\phi} \in \mathscr{C} .
$$

The assumption $s>3 / p+1$ is crucial for $F$ to be of class $C^{1}$. Indeed, it follows from an application of the $\omega$-lemma (cf. [MH, Chapter 3, Theorem 1.13]; [Va, Chapter II, Section 4]) that the mappings

$$
\begin{gathered}
\boldsymbol{H}^{s-1, p}\left(\Omega, \mathbf{R}^{3} \times \mathbf{R}^{3}\right) \rightarrow \boldsymbol{H}^{s-1, p}(\Omega) \\
\nabla \boldsymbol{\nabla} \mapsto \hat{P}_{i j}(\nabla \boldsymbol{\phi})
\end{gathered}
$$

are of class $C^{1}$ if $s \geq 2$ and $s>3 / p+1$.

Now we can state our main existence and uniqueness theorem for problem (1.1) of nonlinear elastostatics (cf. [MH, Chapter 6, Theorem 4.2]; [Va, Chapter IV, Theorem 4.5]):

MAIn Theorem. Let $1<p<\infty, s>3 / p+1$ and $s \geq 2$. We assume that the following three conditions $(\mathrm{P}),(\mathrm{S})$ and $(\mathrm{A})$ are satisfied:

(P) $\hat{\boldsymbol{P}}(\boldsymbol{\nabla} \dot{\boldsymbol{\phi}})=\mathbf{0}$ when $\stackrel{\circ}{\boldsymbol{\phi}}=\boldsymbol{I}_{\Omega}$ (the identity map on $\Omega$ ).

(S) The elasticity tensor $\stackrel{\circ}{\mathbf{A}}(X)=\left(\partial^{2} W / \partial \boldsymbol{F} \partial \boldsymbol{F}\right)(X)$ evaluated at $\stackrel{\circ}{\boldsymbol{\phi}}=\boldsymbol{I}_{\Omega}$ enjoys the property of symmetry

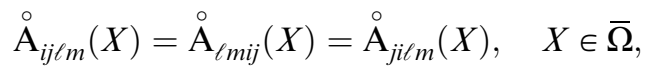

and is uniformly pointwise stable, that is, there is a constant $\eta>0$ such that 


$$
\frac{1}{2} \boldsymbol{e} \cdot \stackrel{\AA}{\mathbf{A}}(X) \cdot \boldsymbol{e} \geq \eta\|\boldsymbol{e}\|^{2}, \quad X \in \bar{\Omega},
$$

for all symmetric two tensors $\boldsymbol{e}$.

(A) $0 \leq \alpha(X) \leq 1$ on $\partial \Omega$, but $\alpha(X) \not \equiv 1$ on $\partial \Omega$.

Then there exist a neighborhood $\mathscr{U}$ of the configuration $\stackrel{\circ}{\boldsymbol{\phi}}$ in $\boldsymbol{H}^{s, p}\left(\Omega, \mathbf{R}^{3}\right)$ and a neighborhood $\mathscr{V}$ of the point

$$
\left(-\operatorname{DIV} \hat{\boldsymbol{P}}(\boldsymbol{\nabla} \dot{\boldsymbol{\phi}}), \alpha(X) \hat{\boldsymbol{P}}(\nabla \stackrel{\circ}{\boldsymbol{\phi}}) \cdot \boldsymbol{N}+\left.(1-\alpha(X)) \stackrel{\circ}{\phi}\right|_{\partial \Omega}\right)
$$

in $\boldsymbol{H}^{s-2, p}\left(\boldsymbol{\Omega}, \mathbf{R}^{3}\right) \times \boldsymbol{B}_{(\alpha)}^{s-1-1 / p, p}\left(\partial \Omega, \mathbf{R}^{3}\right)$ such that the map $F: \mathscr{U} \rightarrow \mathscr{V}$ is one-to-one and onto.

Condition $(\mathrm{P})$ implies that the undeformed state is stress free. We remark that the first Piola-Kirchhoff stress tensors in Examples 1.1 and 1.2 satisfy condition (P). Condition (A) implies that our boundary condition (1.2) is not equal to the pure traction boundary condition. It should be noticed (cf. [MH, Chapter 7, Section 7.3]) that the pure traction problem may have non-unique solutions even for small loads and near a stress free state.

Rephrased, Main Theorem asserts that if the linearized problem is uniformly pointwise stable, then, for slight perturbations of the load or boundary conditions from their values at the undeformed state, the nonlinear problem (1.1) has a unique solution $\phi$ near $\stackrel{\circ}{\phi}=I_{\Omega}$.

For the Hencky-Nadai elasto-plastic material, we have the following result (cf. [Di, Théorème 2]):

THEOREM 1.1. Let $1<p<\infty, s>3 / p+1$ and $s \geq 2$. We assume that the following two conditions (B) and (A) are satisfied:

(B) $g(0)>0$ and $K>0$.

(A) $0 \leq \alpha(X) \leq 1$ on $\partial \Omega$, but $\alpha(X) \not \equiv 1$ on $\partial \Omega$.

Then condition (S) is satisfied and so Main Theorem applies.

For the Saint Venant-Kirchhoff isotropic material, we have the following result (cf. [Ci, Theorem 6.7-1]):

THEOREM 1.2. Let $1<p<\infty, s>3 / p+1$ and $s \geq 2$. We assume that the following two conditions (C) and (A) are satisfied:

(C) There exist constants $c_{1}>0$ and $c_{2}>0$ such that 


$$
\begin{cases}\mu(X) \geq c_{1} & \text { on } \bar{\Omega} \\ \lambda(X)+\frac{2}{3} \mu(X) \geq c_{2} & \text { on } \bar{\Omega}\end{cases}
$$

(A) $0 \leq \alpha(X) \leq 1$ on $\partial \Omega$, but $\alpha(X) \not \equiv 1$ on $\partial \Omega$.

Then condition (S) is satisfied and so Main Theorem applies.

REMARK 1.1. Theorem 1.2 contains [Ci, Theorem 6.4-1] as a special case if we take $s=2, p>3$ and $\alpha(X) \equiv 0$ on $\partial \Omega$ (the pure displacement problem).

The rest of this paper is organized as follows.

In Chapter 2 we present a brief description of the basic concepts and results of the $L^{p}$ theory of pseudo-differential operators which may be considered as a generalization of the classical potential theory. This forms a functional analytic background for the proof of Main Theorem.

In Chapter 3 we linearize problem (1.1) as follows:

$$
\begin{cases}\operatorname{DIV}(\stackrel{\circ}{\mathbf{A}} \cdot \nabla \boldsymbol{V})=-\operatorname{DIV} \hat{\boldsymbol{P}}(\stackrel{\circ}{\boldsymbol{F}})-\boldsymbol{B} & \text { in } \boldsymbol{\Omega}, \\ \alpha(X)(\stackrel{\circ}{\mathbf{A}} \cdot \boldsymbol{\nabla} \boldsymbol{V} \cdot \boldsymbol{N})+(1-\alpha(X)) \boldsymbol{V} & \\ \quad=\boldsymbol{\tau}-\alpha(X) \hat{\boldsymbol{P}}(\stackrel{\circ}{\boldsymbol{F}}) \cdot \boldsymbol{N}-(1-\alpha(X)) \stackrel{\circ}{\boldsymbol{\phi}} & \text { on } \partial \boldsymbol{\Omega},\end{cases}
$$

where

$$
\stackrel{\circ}{\mathbf{A}}:=\frac{\partial \hat{\boldsymbol{P}}}{\partial \boldsymbol{F}}(\stackrel{\circ}{\boldsymbol{F}}), \quad \stackrel{\circ}{\boldsymbol{F}}:=\nabla \stackrel{\circ}{\boldsymbol{\phi}},
$$

is the first elasticity tensor evaluated at the given configuration $\stackrel{\circ}{\text {. }}$

Therefore, we are reduced to the study of a problem of linear elastostatics for the unknown vector function $v$ :

$$
\begin{cases}\boldsymbol{A} \boldsymbol{v}:=\operatorname{div}(\mathbf{a}(x) \cdot \nabla \boldsymbol{v})=\boldsymbol{f} & \text { in } \Omega \\ \boldsymbol{B}_{\alpha} \boldsymbol{v}:=\alpha(x)(\mathbf{a}(x) \cdot \nabla \boldsymbol{v} \cdot \boldsymbol{n})+(1-\alpha(x)) \boldsymbol{v}=\boldsymbol{\varphi} & \text { on } \partial \Omega\end{cases}
$$

where $\mathbf{a}(x)$ is a smooth elasticity tensor and $\boldsymbol{n}$ is the unit outward normal to the boundary $\partial \Omega$.

In Chapters 4 through 7 we study the linearized problem (1.4) in the framework of Sobolev spaces of $L^{p}$ type, by using the $L^{p}$ theory of pseudodifferential operators. Our fundamental existence and uniqueness theorem for problem (1.4) is stated as Theorem 3.1 in Chapter 3. This fundamental theorem (Theorem 3.1) is an essential step in the proof of Main Theorem, and is proved in a series of theorems (Theorems 5.1, 6.1 and 7.1). 
Step 1: In Chapter 4 we show that problem (1.4) can be reduced to the study of a $3 \times 3$ matrix-valued, pseudo-differential operator on the boundary. We explain more precisely the idea of our approach to problem (1.4).

Step 1-a: First, we consider the pure displacement problem (Dirichlet problem)

$$
\begin{cases}\boldsymbol{A} \boldsymbol{v}=\operatorname{div}(\mathbf{a}(x) \cdot \nabla \boldsymbol{v})=\boldsymbol{f} & \text { in } \Omega \\ \boldsymbol{v}=\boldsymbol{\varphi} & \text { on } \partial \Omega\end{cases}
$$

The existence and uniqueness theorem for problem (D) is well established in the framework of Sobolev spaces of $L^{p}$ type (Theorem 4.1). Thus we can introduce the Poisson operator

$$
\mathscr{P}: \boldsymbol{B}^{s-1 / p, p}\left(\partial \Omega, \mathbf{R}^{3}\right) \rightarrow \boldsymbol{H}^{s, p}\left(\Omega, \mathbf{R}^{3}\right)
$$

as follows: For any $\boldsymbol{\varphi} \in \boldsymbol{B}^{s-1 / p, p}\left(\partial \Omega, \mathbf{R}^{3}\right)$, the function $\mathscr{P} \boldsymbol{\varphi}$ is the unique solution of the pure displacement problem

$$
\begin{cases}\boldsymbol{A v}=\mathbf{0} & \text { in } \Omega \\ \boldsymbol{v}=\boldsymbol{\varphi} & \text { on } \partial \Omega .\end{cases}
$$

Step 1-b: Next, we consider the following non-degenerate mixed displacementtraction problem:

$$
\begin{cases}\boldsymbol{A} \boldsymbol{v}=\operatorname{div}(\mathbf{a}(x) \cdot \nabla \boldsymbol{v})=\boldsymbol{f} & \text { in } \Omega \\ (\mathbf{a}(x) \cdot \nabla \boldsymbol{v} \cdot \boldsymbol{n})+\boldsymbol{v}=\boldsymbol{\varphi} & \text { on } \partial \Omega\end{cases}
$$

The existence and uniqueness theorem for problem (M) is also well established in the framework of Sobolev spaces of $L^{p}$ type (Theorem 4.2).

Step 1-c: Then, by using problems (D) and (M) we show that problem (1.4) can be reduced to the study of a $3 \times 3$ matrix-valued, pseudo-differential operator on the boundary, which is a generalization of the classical Fredholm integral equation.

Indeed, if we let

$$
\boldsymbol{T}_{\alpha} \boldsymbol{\varphi}:=\boldsymbol{B}_{\alpha}(\mathscr{P} \boldsymbol{\varphi})
$$

then we have the formula

$$
\boldsymbol{T}_{\alpha}=\alpha(x) \boldsymbol{\Pi}+(1-\alpha(x)) \boldsymbol{I},
$$

where

$$
\Pi \boldsymbol{\varphi}=\left.\mathbf{a}(x) \cdot \nabla(\mathscr{P} \boldsymbol{\varphi}) \cdot \boldsymbol{n}\right|_{\partial \Omega} .
$$


It is known that the operator $\boldsymbol{\Pi}$ is a $3 \times 3$ matrix-valued, classical pseudodifferential operator of first order on the boundary $\partial \Omega$; hence the operator $\boldsymbol{T}_{\alpha}$ is a $3 \times 3$ matrix-valued, classical pseudo-differential operator of first order on the boundary $\partial \Omega$.

We can show that problem (1.4) can be reduced to the study of the system $\boldsymbol{T}_{\alpha}$ of pseudo-differential operators on the boundary $\partial \Omega$ (Theorems 4.4 and 4.5). However, it should be emphasized here that the operator $\boldsymbol{T}_{\alpha}=\alpha(x) \boldsymbol{\Pi}+$ $(1-\alpha(x)) \boldsymbol{I}$ is degenerate elliptic at the points $x \in \partial \Omega$ where $\alpha(x)=0$.

Section 4.2 is devoted to the study of the pseudo-differential operator $\boldsymbol{\Pi}$ (and hence $\boldsymbol{T}_{\alpha}$ ) in question. In particular, by using Green's formula (Theorem 4.6) and Korn's inequality (Theorem 4.7) we show that the operator $\boldsymbol{\Pi}$ is strongly elliptic on the boundary $\partial \Omega$ (Theorem 4.8).

Step 2: In Chapter 5 we prove a regularity theorem for problem (1.4). More precisely, we can construct a parametrix $\boldsymbol{S}_{\alpha}$ for the operator $\boldsymbol{T}_{\alpha}$ in the Hörmander class $\boldsymbol{L}_{1,1 / 2}^{0}\left(\partial \Omega, \mathbf{R}^{3}\right)$ (Lemma 5.2), and then apply a Besov-space boundedness theorem (Theorem 2.11) to the parametrix $\boldsymbol{S}_{\alpha}$ to obtain the regularity theorem for problem (1.4) (Theorem 5.1).

Step 3: Chapter 6 is devoted to a uniqueness theorem for problem (1.4) (Theorem 6.1). By using the regularity theorem for problem (1.4) (Theorem 5.1), we prove that the operator

$$
\mathscr{A}_{\alpha}:=\left(\boldsymbol{A}, \boldsymbol{B}_{\alpha}\right): \boldsymbol{H}^{s, p}\left(\boldsymbol{\Omega}, \mathbf{R}^{3}\right) \mapsto \boldsymbol{H}^{s-2, p}\left(\boldsymbol{\Omega}, \mathbf{R}^{3}\right) \times \boldsymbol{B}_{(\alpha)}^{s-1-1 / p, p}\left(\partial \boldsymbol{\Omega}, \mathbf{R}^{3}\right)
$$

is injective. In the proof we make good use of Korn's inequality (Theorem 4.7) to show an inequality of Gårding type for problem (1.4).

Step 4: Chapter 7 is devoted to an existence theorem for problem (1.4) (Theorem 7.1), which is an essential step in the proof of our fundamental theorem (Theorem 3.1).

By the uniqueness theorem (Theorem 6.1), we know that the operator $\mathscr{A}_{\alpha}$ is injective, that is, $\operatorname{dim} N\left(\mathscr{A}_{\alpha}\right)=0$. Hence, in order to prove the surjectivity of $\mathscr{A}_{\alpha}$ (or equivalently codim $R\left(\mathscr{A}_{\alpha}\right)=0$ ) it suffices to show that the index of the operator $\mathscr{A}_{\alpha}$ is equal to zero (Proposition 7.2), that is,

$$
\text { ind } \mathscr{A}_{\alpha}:=\operatorname{dim} N\left(\mathscr{A}_{\alpha}\right)-\operatorname{codim} R\left(\mathscr{A}_{\alpha}\right)=0 \text {. }
$$

Step 4-a: To do this, we replace the operator $\boldsymbol{A}$ by the operator $\boldsymbol{A}-\lambda \boldsymbol{I}$ with $\lambda \geq 0$, and consider instead of problem (1.4) the following boundary value problem: 


$$
\begin{cases}(\boldsymbol{A}-\lambda \boldsymbol{I}) \boldsymbol{v}=\operatorname{div}(\mathbf{a}(x) \cdot \nabla \boldsymbol{v})-\lambda \boldsymbol{v}=\boldsymbol{f} & \text { in } \Omega, \\ \boldsymbol{B}_{\alpha} \boldsymbol{v}=\alpha(x)(\mathbf{a}(x) \cdot \nabla \boldsymbol{v} \cdot \boldsymbol{n})+(1-\alpha(x)) \boldsymbol{v}=\boldsymbol{\varphi} & \text { on } \partial \Omega .\end{cases}
$$

It should be noticed that problem $(1.5)_{\lambda}$ coincides with problem (1.4) when $\lambda=0$.

Step 4-b: To study problem $(1.5)_{\lambda}$, we shall make use of a method essentially due to Agmon $[\mathrm{Ag}]$. This is a technique of treating a spectral parameter $\lambda \boldsymbol{I}$ as a second-order differential operator of an extra variable and relating the old problem to a new one with the additional variable, which we will explain more precisely.

We introduce an auxiliary variable $y$ of the unit circle

$$
S=\mathbf{R} / 2 \pi \mathbf{Z},
$$

and replace the parameter $-\lambda \boldsymbol{I}$ by the second-order differential operator

$$
\frac{\partial^{2}}{\partial y^{2}} \boldsymbol{I}
$$

Namely, we replace the operator $\boldsymbol{A}-\lambda \boldsymbol{I}$ by the operator

$$
\tilde{\boldsymbol{\Lambda}}=\boldsymbol{A}+\frac{\partial^{2}}{\partial y^{2}} \boldsymbol{I}
$$

and consider instead of problem $(1.5)_{\lambda}$ the following boundary value problem:

$$
\begin{cases}\tilde{\boldsymbol{\Lambda}} \tilde{\boldsymbol{v}}=\operatorname{div}(\mathbf{a}(x) \cdot \nabla \tilde{\boldsymbol{v}})+\frac{\partial^{2} \tilde{\boldsymbol{v}}}{\partial y^{2}}=\tilde{\boldsymbol{f}} & \text { in } \boldsymbol{\Omega} \times S, \\ \boldsymbol{B}_{\alpha} \tilde{\boldsymbol{v}}=\alpha(x)(\mathbf{a}(x) \cdot \nabla \tilde{\boldsymbol{v}} \cdot \boldsymbol{n})+(1-\alpha(x)) \tilde{\boldsymbol{v}}=\tilde{\boldsymbol{\varphi}} & \text { on } \partial \boldsymbol{\Omega} \times S .\end{cases}
$$

Then the most fundamental relationship between problems $(1.5)_{\lambda}$ and problem (1.6) is the following (Proposition 7.4):

If the index of problem (1.6) is finite, then there exists a finite subset $K$ of $\mathbf{Z}$ such that problem $(1.5)_{\lambda}$ is uniquely solvable for all $\lambda^{\prime}=\ell^{2}$ satisfying $\ell \in \mathbf{Z} \backslash K$.

The proof of this assertion is given in Section 7.2, due to its length.

Step 4-c: We show that there exists a parametrix $\tilde{\boldsymbol{S}}_{\alpha}$ for the operator $\tilde{\boldsymbol{T}}_{\alpha}$ in the Hörmander class $\boldsymbol{L}_{1,1 / 2}^{0}\left(\partial \boldsymbol{\Omega} \times S, \mathbf{R}^{3}\right)$ (Lemma 7.3). Therefore, by applying the Besov-space boundedness theorem (Theorem 2.11) and Peetre's lemma (Lemma 7.5) to our situation we obtain that the index of problem (1.6) is finite. By Step 4-b, this proves that the index of $\mathscr{A}_{\alpha}$ is equal to zero.

Step 5: Chapter 8 is devoted to the proof of Main Theorem, Theorem 1.1 and Theorem 1.2. By the existence and uniqueness theorem for problem (1.4) (Theorem 3.1), our Main Theorem follows from an application of the inverse 
mapping theorem (Theorem 8.1). In the proof of Theorems 1.1 and 1.2 we calculate explicitly the first elasticity tensor $\stackrel{\circ}{\mathbf{A}}$, and verify that either condition (B) or condition (C) implies condition (S).

In the final Chapter 9 we give two important open problems concerning the boundary value problems of nonlinear elastostatics for future study.

\section{Theory of Pseudo-Differential Operators}

In this chapter we present a brief description of the basic concepts and results of the $L^{p}$ theory of pseudo-differential operators which may be considered as a generalization of the classical potential theory. This forms a functional analytic background for the proof of Main Theorem. For detailed studies of pseudodifferential operators, the reader is referred to Chazarain-Piriou $[\mathrm{CP}]$, Hörmander [Ho3], Kumano-go [Ku] and Taylor [Ty].

\subsection{Function Spaces}

Let $\Omega$ be a bounded domain of Euclidean space $\mathbf{R}^{n}$ with smooth boundary $\partial \Omega$. Its closure $\bar{\Omega}=\Omega \cup \partial \Omega$ is an $n$-dimensional, compact smooth manifold with boundary. Without loss of generality, we may assume that the domain $\Omega$ is a relatively compact open subset of an $n$-dimensional, compact smooth manifold $M$ without boundary (see Figure 2.1). This manifold $M$ is called the double of $\Omega$.

The function spaces we shall treat are the following (cf. $[\mathrm{AF}],[\mathrm{BL}],[\mathrm{Fr}]$, $[\operatorname{Tr}])$ :

(i) The generalized Sobolev spaces $H^{s, p}(\Omega)$ and $H^{s, p}(M)$, consisting of all potentials of order $s$ of $L^{p}$ functions. When $s$ is integral, these spaces coincide with the usual Sobolev spaces $W^{s, p}(\Omega)$ and $W^{s, p}(M)$, respectively.

(ii) The Besov spaces $B^{s, p}(\partial \Omega)$. These are functions spaces defined in terms of the $L^{p}$ modulus of continuity, and enter naturally in connection with boundary value problems.

First, if $1 \leq p<\infty$, we let

$$
\begin{aligned}
L^{p}(\Omega)= & \text { the space of (equivalence classes of) Lebesgue measurable } \\
& \text { functions } u(x) \text { on } \Omega \text { such that }|u(x)|^{p} \text { is integrable on } \Omega .
\end{aligned}
$$

The space $L^{p}(\Omega)$ is a Banach space with the norm

$$
\|u\|_{p}=\left(\int_{\Omega}|u(x)|^{p} d x\right)^{1 / p} .
$$




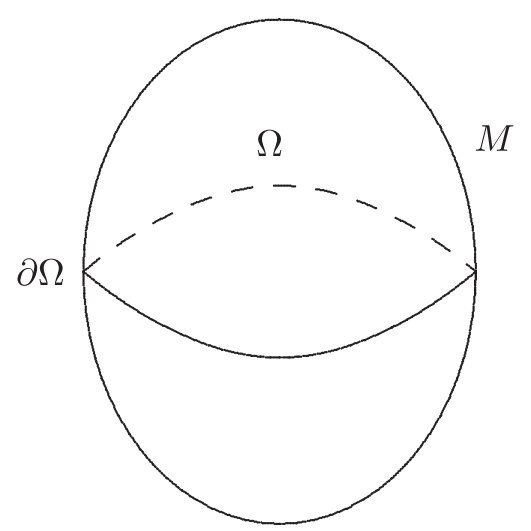

Figure 2.1

For $p=\infty$, we let

$$
\begin{aligned}
L^{\infty}(\Omega)= & \text { the spaceof (equivalence classes of) essentially bounded, } \\
& \text { Lebesgue measurable functions } u(x) \text { on } \Omega .
\end{aligned}
$$

The space $L^{\infty}(\Omega)$ is a Banach space with the norm

$$
\|u\|_{\infty}=\operatorname{ess}_{\sup }|u(x)| .
$$

We recall the basic definitions and facts about the Fourier transform. If $f(x) \in L^{1}\left(\mathbf{R}^{n}\right)$, we define its (direct) Fourier transform $\mathscr{F} f(\xi)$ by the formula

$$
\mathscr{F} f(\xi)=\int_{\mathbf{R}^{n}} e^{-i x \cdot \xi} f(x) d x, \quad \xi=\left(\xi_{1}, \xi_{2}, \ldots, \xi_{n}\right),
$$

where $x \cdot \xi=x_{1} \xi_{1}+x_{2} \xi_{2}+\cdots+x_{n} \xi_{n}$. We also denote $\mathscr{F} f(\xi)$ by $\hat{f}(\xi)$.

Similarly, if $g(\xi) \in L^{1}\left(\mathbf{R}^{n}\right)$, we define its inverse Fourier transform $\mathscr{F}^{*} g(x)$ by the formula

$$
\mathscr{F}^{*} g(x)=\frac{1}{(2 \pi)^{n}} \int_{\mathbf{R}^{n}} e^{i x \cdot \xi} g(\xi) d \xi
$$

We introduce a subspace of $L^{1}\left(\mathbf{R}^{n}\right)$ which is invariant under the Fourier transform. We define the Schwartz space

$$
\begin{aligned}
\mathscr{S}\left(\mathbf{R}^{n}\right)= & \text { the space of smooth functions } \varphi(x) \text { on } \mathbf{R}^{n} \text { such that we have, } \\
& \text { for any non-negative integer } j,
\end{aligned}
$$




$$
p_{j}(\varphi)=\sup _{\substack{x \in \mathbf{R}^{n} \\|\alpha| \leq j}}\left\{\left(1+|x|^{2}\right)^{j / 2}\left|\partial^{\alpha} \varphi(x)\right|\right\}<\infty
$$

We equip the space $\mathscr{S}\left(\mathbf{R}^{n}\right)$ with the topology defined by the countable family $\left\{p_{j}\right\}$ of seminorms. The space $\mathscr{S}\left(\mathbf{R}^{n}\right)$ is a Fréchet space. The Fourier transforms $\mathscr{F}$ and $\mathscr{F}^{*}$ map $\mathscr{S}\left(\mathbf{R}^{n}\right)$ continuously into itself, and $\mathscr{F}^{*}=\mathscr{F}^{*} \mathscr{F}=I$ on $\mathscr{S}\left(\mathbf{R}^{n}\right)$.

Since the injection of $C_{0}^{\infty}\left(\mathbf{R}^{n}\right)$ into $\mathscr{S}\left(\mathbf{R}^{n}\right)$ is continuous, it follows that the dual space $\mathscr{S}^{\prime}\left(\mathbf{R}^{n}\right)$ of $\mathscr{S}\left(\mathbf{R}^{n}\right)$ consists of those distributions $T \in \mathscr{D}^{\prime}\left(\mathbf{R}^{n}\right)$ that have continuous extensions to $\mathscr{S}\left(\mathbf{R}^{n}\right)$. The elements of $\mathscr{S}^{\prime}\left(\mathbf{R}^{n}\right)$ are called tempered distributions on $\mathbf{R}^{n}$. The direct and inverse Fourier transforms can be extended to the space $\mathscr{S}^{\prime}\left(\mathbf{R}^{n}\right)$ by the following formulas:

$$
\begin{aligned}
& \langle\mathscr{F} u, \varphi\rangle=\langle u, \mathscr{F} \varphi\rangle, \quad \varphi \in \mathscr{S}\left(\mathbf{R}^{n}\right) . \\
& \left\langle\mathscr{F}^{*} u, \varphi\right\rangle=\left\langle u, \mathscr{F}^{*} \varphi\right\rangle, \quad \varphi \in \mathscr{S}\left(\mathbf{R}^{n}\right) .
\end{aligned}
$$

Once again, the Fourier transforms $\mathscr{F}$ and $\mathscr{F}^{*}$ map $\mathscr{S}^{\prime}\left(\mathbf{R}^{n}\right)$ continuously into itself, and $\mathscr{F}^{*}=\mathscr{F}^{*} \mathscr{F}=I$ on $\mathscr{S}^{\prime}\left(\mathbf{R}^{n}\right)$.

If $s \in \mathbf{R}$, we define a linear map

$$
J^{s}: \mathscr{S}^{\prime}\left(\mathbf{R}^{n}\right) \rightarrow \mathscr{S}^{\prime}\left(\mathbf{R}^{n}\right)
$$

by the formula

$$
J^{s} u:=\mathscr{F}^{*}\left(\left(1+|\xi|^{2}\right)^{-s / 2} \mathscr{F} u\right), \quad u \in \mathscr{S}^{\prime}\left(\mathbf{R}^{n}\right)
$$

This can be visualized as follows:

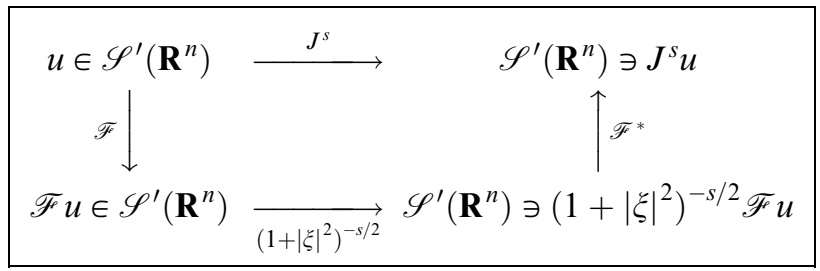

Then it is easy to see that the map $J^{s}$ is an isomorphism of $\mathscr{S}^{\prime}\left(\mathbf{R}^{n}\right)$ onto itself and that its inverse is the map $J^{-s}$. The function $J^{s} u$ is called the Bessel potential of order $s$ of $u$.

(I) Now, if $s \in \mathbf{R}$ and $1<p<\infty$, we let 


$$
H^{s, p}\left(\mathbf{R}^{n}\right)=\text { the image of } L^{p}\left(\mathbf{R}^{n}\right) \text { under the mapping } J^{s} .
$$

We equip $H^{s, p}\left(\mathbf{R}^{n}\right)$ with the norm $\|u\|_{s, p}=\left\|J^{-s} u\right\|_{p}$ for $u \in H^{s, p}\left(\mathbf{R}^{n}\right)$. The space $H^{s, p}\left(\mathbf{R}^{n}\right)$ is called the (generalized) Sobolev space of order $s$.

We list four basic topological properties of $H^{s, p}\left(\mathbf{R}^{n}\right)$ :

(1) The Schwartz space $\mathscr{S}\left(\mathbf{R}^{n}\right)$ is dense in each $H^{s, p}\left(\mathbf{R}^{n}\right)$.

(2) The space $H^{-s, p^{\prime}}\left(\mathbf{R}^{n}\right)$ is the dual space of $H^{s, p}\left(\mathbf{R}^{n}\right)$, where $p^{\prime}=p /(p-1)$ is the exponent conjugate to $p$.

(3) If $s>t$, then we have the inclusions

$$
\mathscr{S}\left(\mathbf{R}^{n}\right) \subset H^{s, p}\left(\mathbf{R}^{n}\right) \subset H^{t, p}\left(\mathbf{R}^{n}\right) \subset \mathscr{S}^{\prime}\left(\mathbf{R}^{n}\right),
$$

with continuous injections.

(4) If $s$ is a non-negative integer, then the space $H^{s, p}\left(\mathbf{R}^{n}\right)$ is isomorphic to the usual Sobolev space $W^{s, p}\left(\mathbf{R}^{n}\right)$, that is, the space $H^{s, p}\left(\mathbf{R}^{n}\right)$ coincides with the space of functions $u(x) \in L^{p}\left(\mathbf{R}^{n}\right)$ such that $D^{\alpha} u(x) \in L^{p}\left(\mathbf{R}^{n}\right)$ for $|\alpha| \leq s$, and the norm $\|\cdot\|_{s, p}$ is equivalent to the norm

$$
\left(\sum_{|\alpha| \leq s} \int_{\mathbf{R}^{n}}\left|D^{\alpha} u(x)\right|^{p} d x\right)^{1 / p}
$$

(II) Next, if $1 \leq p<\infty$, we let

$$
\begin{aligned}
B^{1, p}\left(\mathbf{R}^{n-1}\right)= & \text { the space of (equivalence classes of) functions } \\
& \varphi\left(x^{\prime}\right) \in L^{p}\left(\mathbf{R}^{n-1}\right) \text { for which the integral } \\
& \iint_{\mathbf{R}^{n-1} \times \mathbf{R}^{n-1}} \frac{\left|\varphi\left(x^{\prime}+y^{\prime}\right)-2 \varphi\left(x^{\prime}\right)+\varphi\left(x^{\prime}-y^{\prime}\right)\right|^{p}}{\left|y^{\prime}\right|^{(n-1)+p}} d y^{\prime} d x^{\prime}
\end{aligned}
$$

is finite.

The space $B^{1, p}\left(\mathbf{R}^{n-1}\right)$ is a Banach space with respect to the norm

$$
\begin{aligned}
|\varphi|_{1, p}= & \left(\int_{\mathbf{R}^{n-1}}\left|\varphi\left(x^{\prime}\right)\right|^{p} d x^{\prime}\right. \\
& \left.\quad+\iint_{\mathbf{R}^{n-1} \times \mathbf{R}^{n-1}} \frac{\left|\varphi\left(x^{\prime}+y^{\prime}\right)-2 \varphi\left(x^{\prime}\right)+\varphi\left(x^{\prime}-y^{\prime}\right)\right|^{p}}{\left|y^{\prime}\right|^{n-1+p}} d y^{\prime} d x^{\prime}\right)^{1 / p} .
\end{aligned}
$$

If $p=\infty$, we let 


$$
\begin{aligned}
B^{1, \infty}\left(\mathbf{R}^{n-1}\right)= & \text { the space of (equivalence classes of) functions } \\
& \varphi\left(x^{\prime}\right) \in L^{\infty}\left(\mathbf{R}^{n-1}\right) \text { for which the quantity } \\
& \sup _{\left|y^{\prime}\right|>0} \frac{\left\|\varphi\left(\cdot+y^{\prime}\right)-2 \varphi(\cdot)+\varphi\left(\cdot-y^{\prime}\right)\right\|_{\infty}}{\left|y^{\prime}\right|} \text { is finite. }
\end{aligned}
$$

The space $B^{1, \infty}\left(\mathbf{R}^{n-1}\right)$ is a Banach space with respect to the norm

$$
|\varphi|_{1, \infty}=\|\varphi\|_{\infty}+\sup _{\left|y^{\prime}\right|>0} \frac{\left\|\varphi\left(\cdot+y^{\prime}\right)-2 \varphi(\cdot)+\varphi\left(\cdot-y^{\prime}\right)\right\|_{\infty}}{\left|y^{\prime}\right|} .
$$

If $s \in \mathbf{R}$ and $1 \leq p \leq \infty$, we let

$B^{s, p}\left(\mathbf{R}^{n-1}\right)=$ the image of $B^{1, p}\left(\mathbf{R}^{n-1}\right)$ under the mapping $J^{\prime s-1}$, where $J^{\prime s-1}$ is the Bessel potential of order $s-1$ on $\mathbf{R}^{n-1}$.

We equip the space $B^{s, p}\left(\mathbf{R}^{n-1}\right)$ with the norm $|\varphi|_{s, p}=\left|J^{\prime-s+1} \varphi\right|_{1, p}$ for $\varphi \in B^{s, p}\left(\mathbf{R}^{n-1}\right)$. The space $B^{s, p}\left(\mathbf{R}^{n-1}\right)$ is called the Besov space of order $s$.

We list four basic topological properties of $B^{s, p}\left(\mathbf{R}^{n-1}\right)$ :

(1) The Schwartz space $\mathscr{S}\left(\mathbf{R}^{n-1}\right)$ is dense in each $B^{s, p}\left(\mathbf{R}^{n-1}\right)$.

(2) The space $B^{-s, p^{\prime}}\left(\mathbf{R}^{n-1}\right)$ is the dual space of $B^{s, p}\left(\mathbf{R}^{n-1}\right)$, where $p^{\prime}=$ $p /(p-1)$ is the exponent conjugate to $p$.

(3) If $s>t$, then we have the inclusions

$$
\mathscr{S}\left(\mathbf{R}^{n-1}\right) \subset B^{s, p}\left(\mathbf{R}^{n-1}\right) \subset B^{t, p}\left(\mathbf{R}^{n-1}\right) \subset \mathscr{S}^{\prime}\left(\mathbf{R}^{n-1}\right),
$$

with continuous injections.

(4) If $1 \leq p<\infty$ and if $s=m+\sigma$ with a non-negative integer $m$ and $0<\sigma<1$, then the Besov space $B^{s, p}\left(\mathbf{R}^{n-1}\right)$ coincides with the space of functions $\varphi\left(x^{\prime}\right) \in H^{m, p}\left(\mathbf{R}^{n-1}\right)$ such that, for $|\alpha|=m$, the integral (Slobodeckii seminorm)

$$
\iint_{\mathbf{R}^{n-1} \times \mathbf{R}^{n-1}} \frac{\left|D^{\alpha} \varphi\left(x^{\prime}\right)-D^{\alpha} \varphi\left(y^{\prime}\right)\right|^{p}}{\left|x^{\prime}-y^{\prime}\right|^{n-1+p \sigma}} d x^{\prime} d y^{\prime}
$$

is finite. Furthermore, the norm $|\varphi|_{s, p}$ is equivalent to the norm

$$
\left(\sum_{|\alpha| \leq m} \int_{\mathbf{R}^{n-1}}\left|D^{\alpha} \varphi\left(x^{\prime}\right)\right|^{p} d x^{\prime}+\sum_{|\alpha|=m} \iint_{\mathbf{R}^{n-1} \times \mathbf{R}^{n-1}} \frac{\left|D^{\alpha} \varphi\left(x^{\prime}\right)-D^{\alpha} \varphi\left(y^{\prime}\right)\right|^{p}}{\left|x^{\prime}-y^{\prime}\right|^{n-1+p \sigma}} d x^{\prime} d y^{\prime}\right)^{1 / p} .
$$

Now we define the generalized Sobolev spaces $H^{s, p}(\Omega), H^{s, p}(M)$ and the Besov spaces $B^{s, p}(\partial \Omega)$ for arbitrary values of $s$. 
For each $s \in \mathbf{R}$, we define the Sobolev space

$H^{s, p}(\Omega)=$ the space of restrictions to $\Omega$ of functions in $H^{s, p}\left(\mathbf{R}^{n}\right)$.

We equip the space $H^{s, p}(\Omega)$ with the norm

$$
\|u\|_{s, p}=\inf \left\{\|U\|_{s, p}: U \in H^{s, p}\left(\mathbf{R}^{n}\right),\left.U\right|_{\Omega}=u\right\} .
$$

The space $H^{s, p}(\Omega)$ is a Banach space with respect to the norm $\|\cdot\|_{s, p}$. We remark that

$$
H^{0, p}(\Omega)=L^{p}(\Omega) ; \quad\|\cdot\|_{0, p}=\|\cdot\|_{p} .
$$

The Sobolev spaces $H^{s, p}(M)$ are defined to be locally the spaces $H^{s, p}\left(\mathbf{R}^{n}\right)$, upon using local coordinate systems flattening out $M$, together with a partition of unity. The Besov spaces $B^{s, p}(\partial \Omega)$ are defined similarly, with $H^{s, p}\left(\mathbf{R}^{n}\right)$ replaced by $B^{s, p}\left(\mathbf{R}^{n-1}\right)$. The norms of $H^{s, p}(M)$ and $B^{s, p}(\partial \Omega)$ will be denoted respectively by $\|\cdot\|_{s, p}$ and $|\cdot|_{s, p}$.

We state two important theorems that will be used in the study of boundary value problems in the framework of Sobolev spaces of $L^{p}$ type (see $[\mathrm{AF}],[\mathrm{BL}]$, $[\mathrm{St}],[\mathrm{Tr}])$ :

(I) (The trace theorem) Let $1<p<\infty$. Then the trace map

$$
\begin{aligned}
\rho: H^{s, p}(\Omega) & \rightarrow B^{s-1 / p, p}(\partial \Omega) \\
u & \left.\mapsto u\right|_{\partial \Omega}
\end{aligned}
$$

is continuous for all $s>1 / p$, and is surjective.

(II) (The Rellich-Kondrachov theorem) If $s>t$, then the injections

$$
\begin{gathered}
H^{s, p}(M) \rightarrow H^{t, p}(M), \\
B^{s, p}(\partial \Omega) \rightarrow B^{t, p}(\partial \Omega)
\end{gathered}
$$

are both compact (or completely continuous).

Finally, we introduce a space of distributions on $\Omega$ which behave locally just like the distributions in $H^{s, p}\left(\mathbf{R}^{n}\right)$ :

$$
\begin{aligned}
H_{\text {loc }}^{s, p}(\Omega)= & \text { the space of distributions } u \in \mathscr{D}^{\prime}(\Omega) \text { such that } \\
& \varphi u \in H^{s, p}\left(\mathbf{R}^{n}\right) \text { for all } \varphi \in C_{0}^{\infty}(\Omega) .
\end{aligned}
$$

We equip the localized Sobolev space $H_{\text {loc }}^{s, p}(\Omega)$ with the topology defined by the seminorms $u \mapsto\|\varphi u\|_{s, p}$ as $\varphi$ ranges over $C_{0}^{\infty}(\Omega)$. It is easy to verify that $H_{\text {loc }}^{s, p}(\Omega)$ is a Fréchet space. The localized Besov space $B_{\text {loc }}^{s, p}(\partial \Omega)$ is defined similarly, with $H^{s, p}\left(\mathbf{R}^{n}\right)$ replaced by $B^{s, p}\left(\mathbf{R}^{n-1}\right)$. 


\subsection{Pseudo-Differential Operators}

This section is devoted to a brief description of the basic concepts and results of the $L^{p}$ theory of pseudo-differential operators - a modern theory of classical potentials.

2.2.1 Symbol Classes. Let $\Omega$ be an open subset of $\mathbf{R}^{n}$. If $m \in \mathbf{R}$ and $0 \leq \delta<\rho \leq 1$, we let

$S_{\rho, \delta}^{m}\left(\Omega \times \mathbf{R}^{N}\right)=$ the set of all functions $a(x, \theta) \in C^{\infty}\left(\Omega \times \mathbf{R}^{N}\right)$ with the property that, for any compact $K \subset \Omega$ and any multi-indices $\alpha, \beta$, there exists a constant $C_{K, \alpha, \beta}>0$ such that we have, for all $x \in K$ and all $\theta \in \mathbf{R}^{N}$,

$$
\left|\partial_{\theta}^{\alpha} \partial_{x}^{\beta} a(x, \theta)\right| \leq C_{K, \alpha, \beta}(1+|\theta|)^{m-\rho|\alpha|+\delta|\beta|} .
$$

The elements of $S_{\rho, \delta}^{m}\left(\Omega \times \mathbf{R}^{N}\right)$ are called symbols of order $m$. We drop the $\Omega \times \mathbf{R}^{N}$ and use $S_{\rho, \delta}^{m}$ when the context is clear.

EXAMPLES 2.1. (1) A polynomial $p(x, \xi)=\sum_{|\alpha| \leq m} a_{\alpha}(x) \xi^{\alpha}$ of order $m$ with coefficients in $C^{\infty}(\Omega)$ is in $S_{1,0}^{m}\left(\Omega \times \mathbf{R}^{n}\right)$.

(2) If $m \in \mathbf{R}$, the function

$$
\Omega \times \mathbf{R}^{n} \ni(x, \xi) \mapsto\left(1+|\xi|^{2}\right)^{m / 2}
$$

is in $S_{1,0}^{m}\left(\Omega \times \mathbf{R}^{n}\right)$.

(3) A function $a(x, \theta) \in C^{\infty}\left(\Omega \times\left(\mathbf{R}^{N} \backslash\{0\}\right)\right.$ is said to be positively homogeneous of degree $m$ in $\theta$ if it satisfies the condition

$$
a(x, t \theta)=t^{m} a(x, \theta), \quad t>0 .
$$

If $a(x, \theta)$ is positively homogeneous of degree $m$ in $\theta$ and if $\varphi(\theta)$ is a smooth function such that $\varphi(\theta)=0$ for $|\theta| \leq 1 / 2$ and $\varphi(\theta)=1$ for $|\theta| \geq 1$, then the function $\varphi(\theta) a(x, \theta)$ is in the class $S_{1,0}^{m}\left(\Omega \times \mathbf{R}^{N}\right)$.

If $K$ is a compact subset of $\Omega$ and $j$ is a non-negative integer, we define a seminorm $p_{K, j, m}$ on $S_{\rho, \delta}^{m}\left(\Omega \times \mathbf{R}^{N}\right)$ by the formula

$$
S_{\rho, \delta}^{m}\left(\Omega \times \mathbf{R}^{N}\right) \ni a \mapsto p_{K, j, m}(a)=\sup _{\substack{x \in K^{N} \\ \theta \in \mathbf{R}^{N} \\|\alpha| \leq j}} \frac{\left|\partial_{\theta}^{\alpha} \partial_{x}^{\beta} a(x, \theta)\right|}{(1+|\theta|)^{m-\rho|\alpha|+\delta|\beta|}}
$$


We equip the space $S_{\rho, \delta}^{m}\left(\Omega \times \mathbf{R}^{N}\right)$ with the topology defined by the family $\left\{p_{K, j, m}\right\}$ of seminorms where $K$ ranges over all compact subsets of $\Omega$ and $j=0,1, \ldots$ The space $S_{\rho, \delta}^{m}\left(\Omega \times \mathbf{R}^{N}\right)$ is a Fréchet space.

We set

$$
S^{-\infty}\left(\Omega \times \mathbf{R}^{N}\right)=\bigcap_{m \in \mathbf{R}} S_{\rho, \delta}^{m}\left(\Omega \times \mathbf{R}^{N}\right) .
$$

The next theorem gives a meaning to a formal sum of symbols of decreasing order:

THEOREM 2.1. Let $a_{j}(x, \theta) \in S_{\rho, \delta}^{m_{j}}\left(\Omega \times \mathbf{R}^{N}\right), m_{j} \downarrow-\infty, j=0,1, \ldots$ Then there exists a symbol $a(x, \theta) \in S_{\rho, \delta}^{m_{0}}\left(\Omega \times \mathbf{R}^{N}\right)$, unique modulo $S^{-\infty}\left(\Omega \times \mathbf{R}^{N}\right)$, such that we have, for all $k>0$,

$$
a(x, \theta)-\sum_{j=0}^{k-1} a_{j}(x, \theta) \in S_{\rho, \delta}^{m_{k}}\left(\Omega \times \mathbf{R}^{N}\right) .
$$

If formula (2.1) holds true, we write

$$
a(x, \theta) \sim \sum_{j=0}^{\infty} a_{j}(x, \theta) .
$$

The formal sum $\sum_{j=0}^{\infty} a_{j}(x, \theta)$ is called an asymptotic expansion of $a(x, \theta)$.

A symbol $a(x, \theta) \in S_{1,0}^{m}\left(\Omega \times \mathbf{R}^{N}\right)$ is said to be classical if there exist smooth functions $a_{j}(x, \theta)$, positively homogeneous of degree $m-j$ in $\theta$ for $|\theta| \geq 1$, such that, for all positive integers $k$,

$$
a(x, \theta)-\sum_{j=0}^{k-1} a_{j}(x, \theta) \in S_{1,0}^{m-k}\left(\Omega \times \mathbf{R}^{N}\right) .
$$

The homogeneous function $a_{0}(x, \theta)$ of degree $m$ is called the principal part of $a(x, \theta)$.

We let

$$
S_{\mathrm{cl}}^{m}\left(\Omega \times \mathbf{R}^{N}\right)=\text { the set of all classical symbols of order } m .
$$

For example, the symbols in Examples 2.1 are all classical.

A symbol $a(x, \theta)$ in $S_{\rho, \delta}^{m}\left(\Omega \times \mathbf{R}^{N}\right)$ is said to be elliptic of order $m$ if, for any compact $K \subset \Omega$, there exists a constant $C_{K}>0$ such that 


$$
|a(x, \theta)| \geq C_{K}(1+|\theta|)^{m}, \quad x \in K,|\theta| \geq \frac{1}{C_{K}} .
$$

There is a simple criterion in the case of classical symbols.

THEOREM 2.2. Let $a(x, \theta)$ be in $S_{\mathrm{cl}}^{m}\left(\Omega \times \mathbf{R}^{N}\right)$ with principal part $a_{0}(x, \theta)$. Then $a(x, \theta)$ is elliptic if and only if we have the condition

$$
a_{0}(x, \theta) \neq 0, \quad x \in \Omega,|\theta|=1 .
$$

2.2.2 Pseudo-Differential Operators. Let $\Omega$ be an open subset of $\mathbf{R}^{n}$. If $\varphi(x, y, \xi)$ is a phase function on $\Omega \times \Omega \times\left(\mathbf{R}^{n} \backslash\{0\}\right)$ such that

$$
\varphi(x, y, \xi)=(x-y) \cdot \xi
$$

we define a first-order differential operator

$$
L:=\frac{1}{i} \frac{1-\rho(\xi)}{2+|x-y|^{2}}\left\{\sum_{j=1}^{n}\left(x_{j}-y_{j}\right) \frac{\partial}{\partial \xi_{j}}+\sum_{k=1}^{n} \frac{\xi_{k}}{|\xi|^{2}} \frac{\partial}{\partial x_{k}}+\sum_{k=1}^{n} \frac{-\xi_{k}}{|\xi|^{2}} \frac{\partial}{\partial y_{k}}\right\}+\rho(\xi),
$$

where $i=\sqrt{-1}$ and $\rho(\xi)$ is a function in $C_{0}^{\infty}\left(\mathbf{R}^{n}\right)$ such that $\rho(\xi)=1$ for $|\xi| \leq 1$. Then it is easy to see that the operator $L$ enjoys the property

$$
L\left(e^{i \varphi}\right)=e^{i \varphi} .
$$

We let

$$
S_{\rho, \delta}^{\infty}\left(\Omega \times \Omega \times \mathbf{R}^{n}\right)=\bigcup_{m \in \mathbf{R}} S_{\rho, \delta}^{m}\left(\Omega \times \Omega \times \mathbf{R}^{n}\right)
$$

and we wish to give a meaning to the integral

$$
I_{\varphi}(a w)=\iint_{\Omega \times \Omega \times \mathbf{R}^{n}} e^{i \varphi(x, y, \xi)} a(x, y, \xi) w(x, y) d x d y d \xi, \quad w \in C_{0}^{\infty}(\Omega \times \Omega),
$$

for each symbol $a(x, y, \xi) \in S_{\rho, \delta}^{\infty}\left(\Omega \times \Omega \times \mathbf{R}^{n}\right)$. If we replace $e^{i \varphi}$ in formula (2.2) by $L\left(e^{i \varphi}\right)$, then a formal integration by parts gives us that

$$
I_{\varphi}(a w)=\iint_{\Omega \times \Omega \times \mathbf{R}^{n}} e^{i \varphi(x, y, \xi)} L^{\prime}(a(x, y, \xi) w(x, y)) d x d y d \xi .
$$

However, the transpose $L^{\prime}$ of $L$ maps $S_{\rho, \delta}^{r}$ continuously into $S_{\rho, \delta}^{r-\eta}$ for all $r \in \mathbf{R}$, where $\eta=\min (\rho, 1-\delta)$. Thus, continuing this process we can reduce the growth of the integrand at infinity until it becomes integrable, and give a meaning to the integral (2.2) for each symbol $a(x, y, \xi) \in S_{\rho, \delta}^{\infty}\left(\Omega \times \Omega \times \mathbf{R}^{n}\right)$. 
More precisely, we have the following:

THEOREM 2.3. (i) The linear functional

$$
S^{-\infty}\left(\Omega \times \Omega \times \mathbf{R}^{n}\right) \ni a \mapsto I_{\varphi}(a w) \in \mathbf{C}
$$

extends uniquely to a linear functional $\ell$ on $S_{\rho, \delta}^{\infty}\left(\Omega \times \Omega \times \mathbf{R}^{n}\right)$ whose restriction to each $S_{\rho, \delta}^{m}\left(\Omega \times \Omega \times \mathbf{R}^{n}\right)$ is continuous. Furthermore, the restriction to $S_{\rho, \delta}^{m}\left(\Omega \times \Omega \times \mathbf{R}^{n}\right)$ of $\ell$ is expressed as the formula

$$
\ell(a)=\iint_{\Omega \times \Omega \times \mathbf{R}^{N}} e^{i \varphi(x, y, \xi)}\left(L^{\prime}\right)^{k}(a(x, y, \xi) w(x, y)) d x d y d \xi,
$$

where $k>(m+N) / \eta$ and $\eta=\min (\rho, 1-\delta)$.

(ii) For any fixed $a(x, y, \xi) \in S_{\rho, \delta}^{m}\left(\Omega \times \Omega \times \mathbf{R}^{n}\right)$, the mapping

$$
C_{0}^{\infty}(\Omega \times \Omega) \ni w \mapsto I_{\varphi}(a w)=\ell(a) \in \mathbf{C}
$$

is a distribution of order $\leq k$ for $k>(m+N) / \eta$.

We call the linear functional $\ell$ on $S_{\rho, \delta}^{\infty}$ an oscillatory integral, but use the standard notation as in formula (2.2). The distribution (2.3) is called the Fourier integral distribution associated with the phase function $\varphi(x, y, \xi)$ and the amplitude $a(x, y, \xi)$, and is denoted as follows:

$$
k(x, y)=\int_{\mathbf{R}^{n}} e^{i \varphi(x, y, \xi)} a(x, y, \xi) d \xi .
$$

The distribution $k(x, y)$ defines a continuous linear operator

$$
A: C_{0}^{\infty}(\Omega) \rightarrow \mathscr{D}^{\prime}(\Omega)
$$

by the formula

$$
\langle A u, v\rangle=\langle k, v \otimes u\rangle, \quad u \in C_{0}^{\infty}(\Omega), v \in C_{0}^{\infty}(\Omega) .
$$

The operator $A$ is called a pseudo-differential operator, and is denoted as follows:

$$
A u(x)=\iint_{\Omega \times \mathbf{R}^{n}} e^{i(x-y) \cdot \xi} a(x, y, \xi) u(y) d y d \xi, \quad u \in C_{0}^{\infty}(\Omega) .
$$

For example, the distribution

$$
k(x, y)=\frac{1}{(2 \pi)^{n}} \int_{\mathbf{R}^{n}} e^{i(x-y) \xi} \frac{1}{\left(1+|\xi|^{2}\right)^{s / 2}} d \xi \in \mathscr{D}^{\prime}\left(\mathbf{R}^{n} \times \mathbf{R}^{n}\right)
$$

defines the Bessel potential $J^{s}=(I-\Delta)^{-s / 2}$ for any $s>0$. 
We summarize three basic properties of the operator $A$ :

(1) A pseudo-differential operator $A$ maps $C_{0}^{\infty}(\Omega)$ continuously into $C^{\infty}(\Omega)$, and extends to a continuous linear operator $A: \mathscr{E}^{\prime}(\Omega) \rightarrow \mathscr{D}^{\prime}(\Omega)$.

(2) The distribution kernel $k_{A}(x, y)$ of a pseudo-differential operator $A$ satisfies the condition

$$
\operatorname{sing} \operatorname{supp} k_{A} \subset\{(x, x): x \in \Omega\},
$$

that is, the kernel $k_{A}(x, y)$ is smooth off the diagonal $\{(x, x): x \in \Omega\}$ in $\Omega \times \Omega$. Here we recall that if $u$ is a distribution on $\Omega$, the singular support of $u$ is the smallest closed subset of $\Omega$ outside of which $u$ is smooth; the singular support of $u$ is denoted by $\operatorname{sing} \operatorname{supp} u$.

(3) $\operatorname{sing} \operatorname{supp} A u \subset \operatorname{sing} \operatorname{supp} u, u \in \mathscr{E}^{\prime}(\Omega)$.

In other words, $A u$ is smooth whenever $u$ is. This property is referred to as the pseudo-local property.

A pseudo-differential operator $A$ is said to be of order $m$ if it is an operator of the form (2.4) with some $a(x, y, \xi) \in S_{\rho, \delta}^{m}\left(\Omega \times \Omega \times \mathbf{R}^{n}\right)$. We let

$$
L_{\rho, \delta}^{m}(\Omega)=\text { the set of all pseudo-differential operators of order } m \text { on } \Omega,
$$

and

$$
L^{-\infty}(\Omega)=\bigcap_{m \in \mathbf{R}} L_{\rho, \delta}^{m}(\Omega)
$$

The next theorem characterizes the class $L^{-\infty}(\Omega)$ :

THEOREM 2.4. The following three conditions (i), (ii) and (iii) are equivalent:

(i) $A \in L^{-\infty}(\Omega)$.

(ii) $A$ is written in the form (2.4) with some $a(x, y, \xi) \in S^{-\infty}\left(\Omega \times \Omega \times \mathbf{R}^{n}\right)$.

(iii) $A$ is a regularizer, or equivalently, its distribution kernel $k_{A}(x, y)$ is a smooth function on $\Omega \times \Omega$.

A continuous linear operator $A: C_{0}^{\infty}(\Omega) \rightarrow \mathscr{D}^{\prime}(\Omega)$ is said to be properly supported if the following two conditions (a) and (b) are satisfied:

(a) For any compact subset $K$ of $\Omega$, there exists a compact subset $K^{\prime}$ of $\Omega$ such that

$$
\operatorname{supp} v \subset K \Rightarrow \operatorname{supp} A v \subset K^{\prime} .
$$

(b) For any compact subset $K^{\prime}$ of $\Omega$, there exists a compact subset $K \supset K^{\prime}$ of $\Omega$ such that 


$$
\operatorname{supp} v \cap K=\varnothing \Rightarrow \operatorname{supp} A v \cap K^{\prime}=\varnothing .
$$

If $A$ is properly supported, then it maps $C_{0}^{\infty}(\Omega)$ continuously into $\mathscr{E}^{\prime}(\Omega)$, and further it extends to a continuous linear operator on $C^{\infty}(\Omega)$ into $\mathscr{D}^{\prime}(\Omega)$.

The next theorem states that every pseudo-differential operator can be written as the sum of a properly supported operator and a regularizer:

THEOREM 2.5. If $A \in L_{\rho, \delta}^{m}(\Omega)$, then we have the formula

$$
A=A_{0}+R
$$

where $A_{0} \in L_{\rho, \delta}^{m}(\Omega)$ is properly supported and $R \in L^{-\infty}(\Omega)$.

If $p(x, \xi) \in S_{\rho, \delta}^{m}\left(\Omega \times \mathbf{R}^{n}\right)$, then the operator $p(x, D)$, defined by the formula

$$
p(x, D) u(x):=\frac{1}{(2 \pi)^{n}} \int_{\mathbf{R}^{n}} e^{i x \cdot \xi} p(x, \xi) \hat{u}(\xi) d \xi, \quad u \in C_{0}^{\infty}(\Omega),
$$

is a pseudo-differential operator of order $m$ on $\Omega$, that is, $p(x, D) \in L_{\rho, \delta}^{m}(\Omega)$.

The next theorem asserts that every properly supported pseudo-differential operator can be reduced to the form (2.5):

THEOREM 2.6. If $A \in L_{\rho, \delta}^{m}(\Omega)$ is properly supported, then we have the formula

$$
p(x, \xi)=e^{-i x \cdot \xi} A\left(e^{i x \cdot \xi}\right) \in S_{\rho, \delta}^{m}\left(\Omega \times \mathbf{R}^{n}\right),
$$

and

$$
A=p(x, D) .
$$

Furthermore, if a $(x, y, \xi) \in S_{\rho, \delta}^{m}\left(\Omega \times \Omega \times \mathbf{R}^{n}\right)$ is an amplitude for $A$, we have the following asymptotic expansion:

$$
\left.p(x, \xi) \sim \sum_{\alpha \geq 0} \frac{1}{\alpha !} \partial_{\xi}^{\alpha} D_{y}^{\alpha}(a(x, y, \xi))\right|_{y=x} .
$$

The function $p(x, \xi)$ is called the complete symbol of $A$.

We extend the notion of a complete symbol to the whole space $L_{\rho, \delta}^{m}(\Omega)$. If $A \in L_{\rho, \delta}^{m}(\Omega)$, we choose a properly supported operator $A_{0} \in L_{\rho, \delta}^{m}(\Omega)$ such that $A-A_{0} \in L^{-\infty}(\Omega)$, and let

$$
\begin{aligned}
\sigma(A):= & \text { the equivalence class of the complete symbol of } A_{0} \text { in } \\
& S_{\rho, \delta}^{m}\left(\Omega \times \mathbf{R}^{n}\right) / S^{-\infty}\left(\Omega \times \mathbf{R}^{n}\right) .
\end{aligned}
$$


By virtue of Theorems 2.4 and 2.5, it follows that $\sigma(A)$ does not depend on the operator $A_{0}$ chosen. The equivalence class $\sigma(A)$ is called the complete symbol of $A$. It is easy to see that the mapping

$$
L_{\rho, \delta}^{m}(\Omega) \ni A \mapsto \sigma(A) \in S_{\rho, \delta}^{m}\left(\Omega \times \mathbf{R}^{n}\right) / S^{-\infty}\left(\Omega \times \mathbf{R}^{n}\right)
$$

induces an isomorphism

$$
L_{\rho, \delta}^{m}(\Omega) / L^{-\infty}(\Omega) \rightarrow S_{\rho, \delta}^{m}\left(\Omega \times \mathbf{R}^{n}\right) / S^{-\infty}\left(\Omega \times \mathbf{R}^{n}\right) .
$$

We shall often identify the complete symbol $\sigma(A)$ with a representative in the class $S_{\rho, \delta}^{m}\left(\Omega \times \mathbf{R}^{n}\right)$ for notational convenience, and call any member of $\sigma(A)$ a complete symbol of $A$.

A pseudo-differential operator $A \in L_{1,0}^{m}(\Omega)$ is said to be classical if its complete symbol $\sigma(A)$ has a representative in the class $S_{\mathrm{cl}}^{m}\left(\Omega \times \mathbf{R}^{n}\right)$.

We let

$L_{\mathrm{cl}}^{m}(\Omega)=$ the set of all classical pseudo-differential operators of order $m$ on $\Omega$.

Then the mapping

$$
L_{\mathrm{cl}}^{m}(\Omega) \ni A \mapsto \sigma(A) \in S_{\mathrm{cl}}^{m}\left(\Omega \times \mathbf{R}^{n}\right) / S^{-\infty}\left(\Omega \times \mathbf{R}^{n}\right)
$$

induces an isomorphism

$$
L_{\mathrm{cl}}^{m}(\Omega) / L^{-\infty}(\Omega) \rightarrow S_{\mathrm{cl}}^{m}\left(\Omega \times \mathbf{R}^{n}\right) / S^{-\infty}\left(\Omega \times \mathbf{R}^{n}\right) .
$$

Also we have the formula

$$
L^{-\infty}(\Omega)=\bigcap_{m \in \mathbf{R}} L_{\mathrm{cl}}^{m}(\Omega)
$$

If $A \in L_{\mathrm{cl}}^{m}(\Omega)$, then the principal part of $\sigma(A)$ has a canonical representative $\sigma_{A}(x, \xi) \in C^{\infty}\left(\Omega \times\left(\mathbf{R}^{n} \backslash\{0\}\right)\right)$ which is positively homogeneous of degree $m$ in the variable $\xi$. The function $\sigma_{A}(x, \xi)$ is called the homogeneous principal symbol of $A$.

The next two theorems assert that the class of pseudo-differential operators forms an algebra closed under the operations of composition of operators and taking the transpose or adjoint of an operator:

THEOREM 2.7. If $A \in L_{\rho, \delta}^{m}(\Omega)$, then its transpose $A^{\prime}$ and its adjoint $A^{*}$ are both in $L_{\rho, \delta}^{m}(\Omega)$, and the complete symbols $\sigma\left(A^{\prime}\right)$ and $\sigma\left(A^{*}\right)$ have respectively the following asymptotic expansions: 


$$
\begin{aligned}
& \sigma\left(A^{\prime}\right)(x, \xi) \sim \sum_{\alpha \geq 0} \frac{1}{\alpha !} \partial_{\xi}^{\alpha} D_{x}^{\alpha}(\sigma(A)(x,-\xi)), \\
& \sigma\left(A^{*}\right)(x, \xi) \sim \sum_{\alpha \geq 0} \frac{1}{\alpha !} \partial_{\xi}^{\alpha} D_{x}^{\alpha}(\overline{\sigma(A)(x, \xi)}) .
\end{aligned}
$$

THEOREM 2.8. If $A \in L_{\rho^{\prime}, \delta^{\prime}}^{m^{\prime}}(\Omega)$ and $B \in L_{\rho^{\prime \prime}, \delta^{\prime \prime}}^{m^{\prime \prime}}(\Omega)$ where $0 \leq \delta^{\prime}<\rho^{\prime \prime} \leq 1$ and if one of them is properly supported, then the composition $A B$ is in $L_{\rho, \delta}^{m^{\prime}+m^{\prime \prime}}(\Omega)$ with $\rho=\min \left(\rho^{\prime}, \rho^{\prime \prime}\right)$ and $\delta=\max \left(\delta^{\prime}, \delta^{\prime \prime}\right)$, and we have the following asymptotic expansion:

$$
\sigma(A B)(x, \xi) \sim \sum_{\alpha \geq 0} \frac{1}{\alpha !} \partial_{\xi}^{\alpha}(\sigma(A)(x, \xi)) \cdot D_{x}^{\alpha}(\sigma(B)(x, \xi))
$$

A pseudo-differential operator $A \in L_{\rho, \delta}^{m}(\Omega)$ is said to be elliptic of order $m$ if its complete symbol $\sigma(A)$ is elliptic of order $m$. By virtue of Theorem 2.2, it follows that a classical pseudo-differential operator $A \in L_{\mathrm{cl}}^{m}(\Omega)$ is elliptic if and only if its homogeneous principal symbol $\sigma_{A}(x, \xi)$ does not vanish on the space $\Omega \times\left(\mathbf{R}^{n} \backslash\{0\}\right)$.

The next theorem states that elliptic operators are the "invertible" elements in the algebra of pseudo-differential operators:

THEOREM 2.9. An operator $A \in L_{\rho, \delta}^{m}(\Omega)$ is elliptic if and only if there exists a properly supported operator $B \in L_{\rho, \delta}^{-m}(\Omega)$ such that

$$
\begin{cases}A B \equiv I & \bmod L^{-\infty}(\Omega), \\ B A \equiv I & \bmod L^{-\infty}(\Omega) .\end{cases}
$$

Such an operator $B$ is called a parametrix for $A$. In other words, a parametrix for $A$ is a two-sided inverse of $A$ modulo $L^{-\infty}(\Omega)$. We observe that a parametrix is unique modulo $L^{-\infty}(\Omega)$.

The next theorem proves the invariance of pseudo-differential operators under change of coordinates:

THEOREM 2.10. Let $\Omega_{1}$ and $\Omega_{2}$ be two open subsets of $\mathbf{R}^{n}$ and $\chi: \Omega_{1} \rightarrow \Omega_{2}$ a $C^{\infty}$ diffeomorphism. If $A \in L_{\rho, \delta}^{m}\left(\Omega_{1}\right)$, where $1-\rho \leq \delta<\rho \leq 1$, then the mapping

$$
\begin{gathered}
A_{\chi}: C_{0}^{\infty}\left(\Omega_{2}\right) \rightarrow C^{\infty}\left(\Omega_{2}\right) \\
v \mapsto A(v \circ \chi) \circ \chi^{-1}
\end{gathered}
$$


is in $L_{\rho, \delta}^{m}\left(\Omega_{2}\right)$, and we have the asymptotic expansion

$$
\left.\sigma\left(A_{\chi}\right)(y, \eta) \sim \sum_{\alpha \geq 0} \frac{1}{\alpha !}\left(\partial_{\xi}^{\alpha} \sigma(A)\right)\left(x,{ }^{t} \chi^{\prime}(x) \cdot \eta\right) \cdot D_{z}^{\alpha}\left(e^{i r(x, z, \eta)}\right)\right|_{z=x}
$$

with

$$
r(x, z, \eta):=\left\langle\chi(z)-\chi(x)-\chi^{\prime}(x) \cdot(z-x), \eta\right\rangle .
$$

Here $x=\chi^{-1}(y), \chi^{\prime}(x)$ is the derivative of $\chi$ at $x$ and ${ }^{t} \chi^{\prime}(x)$ its transpose.

The situation may be represented by the following diagram:

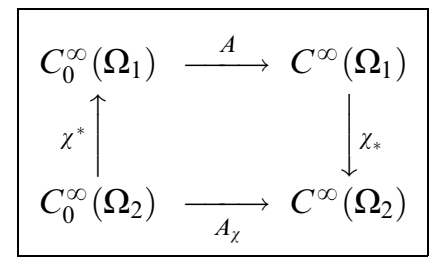

Here $\chi^{*} v=v \circ \chi$ is the pull-back of $v$ by $\chi$ and $\chi_{*} u=u \circ \chi^{-1}$ is the push-forward of $u$ by $\chi$, respectively.

REMARK 2.1. Formula (2.6) shows that

$$
\sigma\left(A_{\chi}\right)(y, \eta) \equiv \sigma(A)\left(x,{ }^{t} \chi^{\prime}(x) \cdot \eta\right) \bmod S_{\rho, \delta}^{m-(\rho-\delta)} .
$$

Note that the mapping

$$
\Omega_{2} \times \mathbf{R}^{n} \ni(y, \eta) \mapsto\left(x,{ }^{t} \chi^{\prime}(x) \cdot \eta\right) \in \Omega_{1} \times \mathbf{R}^{n}
$$

is just a transition map of the cotangent bundle $T^{*}\left(\mathbf{R}^{n}\right)$. This implies that the principal symbol $\sigma_{m}(A)$ of $A \in L_{\rho, \delta}^{m}\left(\mathbf{R}^{n}\right)$ can be invariantly defined on $T^{*}\left(\mathbf{R}^{n}\right)$ when $1-\rho \leq \delta<\rho \leq 1$.

A differential operator of order $m$ with smooth coefficients on $\Omega$ is continuous on $H_{\mathrm{loc}}^{s, p}(\Omega)\left(\operatorname{resp} . B_{\mathrm{loc}}^{s, p}(\Omega)\right)$ into $H_{\mathrm{loc}}^{s-m, p}(\Omega)\left(\operatorname{resp} . B_{\mathrm{loc}}^{s-m, p}(\Omega)\right)$ for all $s \in \mathbf{R}$. This result extends to pseudo-differential operators (cf. [Bo, Theorem 1]; [Ta4, Theorem A.6]):

THEOREM 2.11. Every properly supported operator $A \in L_{1, \delta}^{m}(\Omega), 0 \leq \delta<1$, extends to a continuous linear operator $A: H_{\mathrm{loc}}^{s, p}(\Omega) \rightarrow H_{\mathrm{loc}}^{s-m, p}(\Omega)$ for all $s \in \mathbf{R}$ and all $1<p<\infty$, and also it extends to a continuous linear operator $A: B_{\mathrm{loc}}^{s, p}(\Omega) \rightarrow$ $B_{\mathrm{loc}}^{s-m, p}(\Omega)$ for all $s \in \mathbf{R}$ and all $1 \leq p \leq \infty$. 
Now we can define the concept of a pseudo-differential operator on a manifold, and transfer all the machinery of pseudo-differential operators to manifolds. Let $M$ be an $n$-dimensional, compact smooth manifold without boundary. Theorem 2.10 leads us to the following:

Definition 2.1. Let $1-\rho \leq \delta<\rho \leq 1$. A continuous linear operator $A: C^{\infty}(M) \rightarrow C^{\infty}(M)$ is called a pseudo-differential operator of order $m \in \mathbf{R}$ if it satisfies the following two conditions (i) and (ii):

(i) The distribution kernel $k_{A}(x, y)$ of $A$ is smooth off the diagonal $\{(x, x): x \in M\}$ in $M \times M$.

(ii) For any chart $(U, \chi)$ on $M$, the mapping

$$
\begin{gathered}
A_{\chi}: C_{0}^{\infty}(\chi(U)) \rightarrow C^{\infty}(\chi(U)) \\
u \mapsto A(u \circ \chi) \circ \chi^{-1}
\end{gathered}
$$

belongs to the class $L_{\rho, \delta}^{m}(\chi(U))$.

We let

$L_{\rho, \delta}^{m}(M)=$ the set of all pseudo-differential operators of order $m$ on $M$, and set

$$
L^{-\infty}(M)=\bigcap_{m \in \mathbf{R}} L_{\rho, \delta}^{m}(M)
$$

Some results about pseudo-differential operators on $\mathbf{R}^{n}$ stated above are also true for pseudo-differential operators on $M$. In fact, pseudo-differential operators on $M$ are defined to be locally pseudo-differential operators on $\mathbf{R}^{n}$.

For example, we have the following five results (1) through (5):

(1) A pseudo-differential operator $A$ extends to a continuous linear operator $A: \mathscr{D}^{\prime}(M) \rightarrow \mathscr{D}^{\prime}(M)$.

(2) sing supp $A u \subset \operatorname{sing} \operatorname{supp} u, u \in \mathscr{D}^{\prime}(M)$.

(3) A continuous linear operator $A: C^{\infty}(M) \rightarrow \mathscr{D}^{\prime}(M)$ is a regularizer if and only if it is in the class $L^{-\infty}(M)$.

(4) The class $L_{\rho, \delta}^{m}(M)$ is stable under the operations of composition of operators and taking the transpose or adjoint of an operator.

(5) A pseudo-differential operator $A \in L_{1, \delta}^{m}(M), 0 \leq \delta<1$, extends to a continuous linear operator $A: H^{s, p}(M) \rightarrow H^{s-m, p}(M)$ for all $s \in \mathbf{R}$ and all $1<p<\infty$ and also a continuous linear operator $A: B^{s, p}(M) \rightarrow B^{s-m, p}(M)$ for all $s \in \mathbf{R}$ and all $1 \leq p \leq \infty$, respectively. 
A pseudo-differential operator $A \in L_{1,0}^{m}(M)$ is said to be classical if, for any chart $(U, \chi)$ on $M$, the mapping $A_{\chi}: C_{0}^{\infty}(\chi(U)) \rightarrow C^{\infty}(\chi(U))$ belongs to the class $L_{\mathrm{cl}}^{m}(\chi(U))$.

We let

$L_{\mathrm{cl}}^{m}(M)=$ the set of all classical pseudo-differential operators of order $m$ on $M$.

We observe that

$$
L^{-\infty}(M)=\bigcap_{m \in \mathbf{R}} L_{\mathrm{cl}}^{m}(M)
$$

Let $A \in L_{\mathrm{cl}}^{m}(M)$. If $(U, \chi)$ is a chart on $M$, there is associated a homogeneous principal symbol $\sigma_{A_{\chi}}(x, \xi) \in C^{\infty}\left(\chi(U) \times\left(\mathbf{R}^{n} \backslash\{0\}\right)\right)$. In view of Remark 2.1, by smoothly patching together the functions $\sigma_{A_{x}}(x, \xi)$ we can obtain a smooth function $\sigma_{A}(x, \xi)$ on $T^{*}(M) \backslash\{0\}=\left\{(x, \xi) \in T^{*}(M): \xi \neq 0\right\}$, which is positively homogeneous of degree $m$ in the variable $\xi$. The function $\sigma_{A}(x, \xi)$ is called the homogeneous principal symbol of $A$.

A classical pseudo-differential operator $A \in L_{\mathrm{cl}}^{m}(M)$ is said to be elliptic of order $m$ if its homogeneous principal symbol $\sigma_{A}(x, \xi)$ does not vanish on the bundle $T^{*}(M) \backslash\{0\}$ of non-zero cotangent vectors.

Then we have the following result:

(6) An operator $A \in L_{\mathrm{cl}}^{m}(M)$ is elliptic if and only if there exists a parametrix $B \in L_{\mathrm{cl}}^{-m}(M)$ for $A$ :

$$
\begin{cases}A B \equiv I \quad \bmod L^{-\infty}(M) \\ B A \equiv I \quad \bmod L^{-\infty}(M) .\end{cases}
$$

Let $\Omega$ be an open subset of $\mathbf{R}^{n}$. A properly supported pseudo-differential operator $A$ on $\Omega$ is said to be hypoelliptic if it satisfies the condition

$$
\operatorname{sing} \operatorname{supp} u=\operatorname{sing} \operatorname{supp} A u, \quad u \in \mathscr{D}^{\prime}(\Omega) .
$$

For example, Theorem 2.9 asserts that elliptic operators are hypoelliptic. It should be emphasized that this notion may be transferred to manifolds.

The following criterion for hypoellipticity is due to Hörmander (cf. [Ho2, Theorem 4.2]):

THEOREM 2.12. Let $A=p(x, D) \in L_{\rho, \delta}^{m}(\Omega)$ be properly supported. Assume that, for any compact $K \subset \Omega$ and any multi-indices $\alpha, \beta$, there exist constants $C_{K, \alpha, \beta}>0, C_{K}>0$ and $\mu \in \mathbf{R}$ such that we have, for all $x \in K$ and all $|\xi| \geq C_{K}$, 


$$
\begin{aligned}
& \left|D_{\xi}^{\alpha} D_{x}^{\beta} p(x, \xi)\right| \leq C_{K, \alpha, \beta}|p(x, \xi)|(1+|\xi|)^{-\rho|\alpha|+\delta|\beta|} \\
& \left|p(x, \xi)^{-1}\right| \leq C_{K}(1+|\xi|)^{\mu}
\end{aligned}
$$

Then there exists a parametrix $B \in L_{\rho, \delta}^{\mu}(\Omega)$ for $A$.

REMARK 2.2. It should be emphasized that Theorem 2.12 extends to the class $\boldsymbol{L}_{\rho, \delta}^{m}\left(\Omega, \mathbf{R}^{n}\right)$ of $n \times n$ matrix-valued, pseudo-differential operators on $\Omega$.

\section{Linear Elastostatics}

The process of linearization provides a key link between the linear and nonlinear theories of elasticity. In this chapter we study a linearization of problem (1.1) of nonlinear elastostatics, and state our fundamental existence and uniqueness theorem (Theorem 3.1) for the linearized problem (1.4) in the framework of Sobolev spaces of $L^{p}$ type.

\subsection{Linearization of Nonlinear Elastostatics}

Let $\boldsymbol{B}: \Omega \rightarrow \mathbf{R}^{3}$ be a given body force and $\tau: \partial \Omega \rightarrow \mathbf{R}^{3}$ a given surface force. In this section we study a linearization of problem (1.1) of nonlinear elastostatics for the unknown configuration $\phi$ :

$$
\begin{cases}\operatorname{DIV} \hat{\boldsymbol{P}}(X, \nabla \boldsymbol{\phi}(X))+\boldsymbol{B}(X)=\mathbf{0} & \text { in } \Omega, \\ \alpha(X) \hat{\boldsymbol{P}}(X, \nabla \phi(X)) \cdot \boldsymbol{N}(X)+(1-\alpha(X)) \boldsymbol{\phi}(X)=\boldsymbol{\tau}(X) & \text { on } \partial \Omega .\end{cases}
$$

Componentwise, our problem (1.1) can be written as follows:

$$
\begin{cases}\sum_{j=1}^{3} \frac{\partial}{\partial X_{j}}\left(\hat{P}_{i j}(X, \boldsymbol{\nabla} \phi(X))\right)+B_{i}(X)=0 & \text { in } \Omega, \\ \alpha(X) \sum_{j=1}^{3} \hat{P}_{i j}(X, \boldsymbol{\nabla} \phi(X)) N_{j}(X)+(1-\alpha(X)) \phi_{i}(X)=\tau_{i}(X) & \text { on } \partial \Omega,\end{cases}
$$

where $1 \leq i \leq 3$. Indeed, it suffices to recall that if $\boldsymbol{T}$ is a tensor field

$$
\boldsymbol{T}=\left(\begin{array}{lll}
T_{11} & T_{12} & T_{13} \\
T_{21} & T_{22} & T_{23} \\
T_{31} & T_{32} & T_{33}
\end{array}\right)
$$

then the divergence DIV $\boldsymbol{T}$ is defined by the formula

$$
\text { DIV } \boldsymbol{T}:=\left(\begin{array}{l}
\frac{\partial T_{11}}{\partial X_{1}}+\frac{\partial T_{12}}{\partial X_{2}}+\frac{\partial T_{13}}{\partial X_{3}} \\
\frac{\partial T_{21}}{\partial X_{1}}+\frac{\partial T_{22}}{\partial X_{2}}+\frac{\partial T_{23}}{\partial X_{3}} \\
\frac{\partial T_{31}}{\partial X_{1}}+\frac{\partial T_{32}}{\partial X_{2}}+\frac{\partial T_{33}}{\partial X_{3}}
\end{array}\right) .
$$


It should be noticed that a simple application of Green's formula implies the divergence theorem for tensor fields

$$
\int_{\Omega} \operatorname{DIV} \boldsymbol{T} d x=\int_{\partial \Omega} \boldsymbol{T} \cdot \boldsymbol{N} d a,
$$

where $d a$ is the area element on the boundary $\partial \Omega$.

First, we linearize the nonlinear equations of elastostatics

$$
\operatorname{DIV} \hat{\boldsymbol{P}}(\nabla \boldsymbol{\phi})+\boldsymbol{B}=\mathbf{0} \text { in } \Omega .
$$

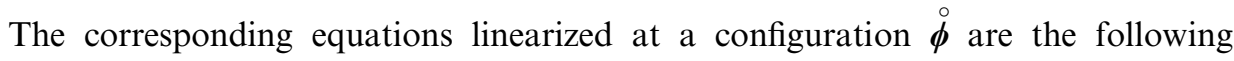
(cf. [MH, Chapter 4, Section 4.2]):

$$
\operatorname{DIV}(\hat{\boldsymbol{P}}(\stackrel{\circ}{\boldsymbol{F}})+\stackrel{\circ}{\mathbf{A}} \cdot \nabla \boldsymbol{V})+\boldsymbol{B}=\mathbf{0} \quad \text { in } \Omega,
$$

where

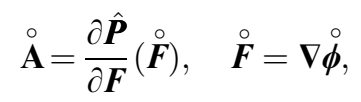

is the first elasticity tensor evaluated at $\stackrel{\circ}{\phi}$.

Similarly, the linearization of the boundary condition

$$
\left\{\begin{array}{l}
\alpha(X) \hat{\boldsymbol{P}}(\boldsymbol{\nabla} \boldsymbol{\phi}) \cdot \boldsymbol{N}+(1-\alpha(X)) \boldsymbol{\phi}=\boldsymbol{\tau} \quad \text { on } \partial \boldsymbol{\Omega}, \\
0 \leq \alpha(X) \leq 1 \quad \text { on } \partial \Omega
\end{array}\right.
$$

about a configuration $\stackrel{\circ}{\phi}$ is the following:

$$
\alpha(X)(\hat{\boldsymbol{P}}(\stackrel{\circ}{\boldsymbol{F}})+\stackrel{\circ}{\mathbf{A}} \cdot \nabla \boldsymbol{V}) \cdot \boldsymbol{N}+(1-\alpha(X))(\stackrel{\circ}{\boldsymbol{\phi}}+\boldsymbol{V})=\boldsymbol{\tau} \quad \text { on } \partial \boldsymbol{\Omega} .
$$

Summing up, we obtain the following linearization of problem (1.1) for the unknown vector function $\boldsymbol{V}$ :

$$
\begin{cases}\operatorname{DIV}(\stackrel{\circ}{\mathbf{A}} \cdot \nabla \boldsymbol{V})=-\operatorname{DIV} \hat{\boldsymbol{P}}(\stackrel{\circ}{\boldsymbol{F}})-\boldsymbol{B} & \text { in } \Omega \\ \alpha(X)(\stackrel{\circ}{\mathbf{A}} \cdot \boldsymbol{\nabla} \boldsymbol{V} \cdot \boldsymbol{N})+(1-\alpha(X)) \boldsymbol{V} & \\ \quad=\boldsymbol{\tau}-\alpha(X) \hat{\boldsymbol{P}}(\stackrel{\circ}{\boldsymbol{F}}) \cdot \boldsymbol{N}-(1-\alpha(X)) \stackrel{\circ}{\boldsymbol{\phi}} & \text { on } \partial \Omega .\end{cases}
$$

\subsection{Existence and Uniqueness Theorem for Problem (1.4)}

In this section we consider problem (1.3) of linear elastostatics in the following form:

$$
\begin{cases}\operatorname{div}(\mathbf{a}(x) \cdot \nabla \boldsymbol{v})=\boldsymbol{f} & \text { in } \boldsymbol{\Omega} \\ \alpha(x)(\mathbf{a}(x) \cdot \nabla \boldsymbol{v} \cdot \boldsymbol{n})+(1-\alpha(x)) \boldsymbol{v}=\boldsymbol{\varphi} & \text { on } \partial \boldsymbol{\Omega}\end{cases}
$$


where $\mathbf{a}(x)$ is a smooth elasticity tensor and $\boldsymbol{n}$ is the unit outward normal to the boundary $\partial \Omega$. Componentwise, these equations can be written as follows:

$$
\begin{cases}\sum_{j=1}^{3} \frac{\partial}{\partial x_{j}}\left(\sum_{\ell, m=1}^{3} a_{i j \ell m}(x) \frac{\partial v_{\ell}}{\partial x_{m}}\right)=f_{i}(x) & \text { in } \Omega, \\ \alpha(x) \sum_{j=1}^{3}\left(\sum_{\ell, m=1}^{3} a_{i j \ell m}(x) \frac{\partial v_{\ell}}{\partial x_{m}}\right) n_{j}(x)+(1-\alpha(x)) v_{i}(x)=\varphi_{i}(x) & \text { on } \partial \Omega,\end{cases}
$$

where $1 \leq i \leq 3$.

We study problem (1.4) in the framework of Sobolev spaces of $L^{p}$ type, by using the $L^{p}$ theory of pseudo-differential operators.

If $s \in \mathbf{R}$ and $1<p<\infty$, we let

$$
\begin{aligned}
& \boldsymbol{H}^{s, p}\left(\Omega, \mathbf{R}^{3}\right)=\text { the Banach space of all } H^{s, p} \text { vector functions } \boldsymbol{u}, \\
& \boldsymbol{B}^{s, p}\left(\partial \Omega, \mathbf{R}^{3}\right)=\text { the Banach space of all } B^{s, p} \text { vector functions } \boldsymbol{\phi} .
\end{aligned}
$$

We introduce a subspace of $B^{s, p}\left(\partial \Omega, \mathbf{R}^{3}\right)$ which is associated with the boundary condition

$$
\alpha(x)(\mathbf{a}(x) \cdot \nabla \boldsymbol{v} \cdot \boldsymbol{n})+(1-\alpha(x)) \boldsymbol{v}=\boldsymbol{\varphi} \quad \text { on } \partial \Omega .
$$

If $s \in \mathbf{R}$ and $1<p<\infty$, we let

$$
\begin{gathered}
\boldsymbol{B}_{(\alpha)}^{s, p}\left(\partial \Omega, \mathbf{R}^{3}\right)=\left\{\boldsymbol{\phi}=\alpha(x) \boldsymbol{\phi}_{1}+(1-\alpha(x)) \boldsymbol{\phi}_{2}: \boldsymbol{\phi}_{1} \in \boldsymbol{B}^{s, p}\left(\partial \boldsymbol{\Omega}, \mathbf{R}^{3}\right),\right. \\
\left.\boldsymbol{\phi}_{2} \in \boldsymbol{B}^{s+1, p}\left(\partial \Omega, \mathbf{R}^{3}\right)\right\},
\end{gathered}
$$

and define the norm

$$
|\boldsymbol{\phi}|_{\alpha ; s, p}=\inf \left\{\left|\phi_{1}\right|_{s, p}+\left|\phi_{2}\right|_{s+1, p}: \boldsymbol{\phi}=\alpha(x) \boldsymbol{\phi}_{1}+(1-\alpha(x)) \boldsymbol{\phi}_{2}\right\} .
$$

Then it is easy to verify (see [Ta2, Lemma 4.7]) that the space $\boldsymbol{B}_{(\alpha)}^{s, p}\left(\partial \boldsymbol{\Omega}, \mathbf{R}^{3}\right)$ is a Banach space with the norm $|\cdot|_{\alpha ; s, p}$. Furthermore, we remark that $\boldsymbol{B}_{(\alpha)}^{s, p}\left(\partial \Omega, \mathbf{R}^{3}\right)= \begin{cases}\boldsymbol{B}^{s+1, p}\left(\partial \boldsymbol{\Omega}, \mathbf{R}^{3}\right) & \text { if } \alpha(x) \equiv 0 \text { on } \partial \boldsymbol{\Omega} \text { (the pure displacement case), } \\ \boldsymbol{B}^{s, p}\left(\partial \Omega, \mathbf{R}^{3}\right) & \text { if } \alpha(x) \equiv 1 \text { on } \partial \boldsymbol{\Omega} \text { (the pure traction case), }\end{cases}$ and we have, for general $\alpha(x)$, the continuous injections

$$
\boldsymbol{B}^{s+1, p}\left(\partial \boldsymbol{\Omega}, \mathbf{R}^{3}\right) \subset \boldsymbol{B}_{(\alpha)}^{s, p}\left(\partial \Omega, \mathbf{R}^{3}\right) \subset \boldsymbol{B}^{s, p}\left(\partial \boldsymbol{\Omega}, \mathbf{R}^{3}\right) .
$$

Now we let

$$
\begin{aligned}
& \boldsymbol{A v}:=\operatorname{div}(\mathbf{a}(x) \cdot \boldsymbol{\nabla} \boldsymbol{v}), \\
& \boldsymbol{B}_{\alpha} \boldsymbol{v}:=\alpha(x)(\mathbf{a}(x) \cdot \boldsymbol{\nabla} \boldsymbol{v} \cdot \boldsymbol{n})+\left.(1-\alpha(x)) \boldsymbol{v}\right|_{\partial \Omega},
\end{aligned}
$$


and associate with problem (1.4) a linear operator

$$
\left(\boldsymbol{A}, \boldsymbol{B}_{\alpha}\right): \boldsymbol{H}^{s, p}\left(\Omega, \mathbf{R}^{3}\right) \mapsto \boldsymbol{H}^{s-2, p}\left(\Omega, \mathbf{R}^{3}\right) \times \boldsymbol{B}_{(\alpha)}^{s-1-1 / p, p}\left(\partial \Omega, \mathbf{R}^{3}\right) .
$$

Then it is easy to verify that the operator $\left(\boldsymbol{A}, \boldsymbol{B}_{\alpha}\right)$ is continuous, for $s>1+1 / p$.

Our fundamental result is the following existence and uniqueness theorem for problem (1.4) (cf. [It1, Theorem I]):

THEOREM 3.1. Let $1<p<\infty$ and $s>1 / p+1$. We assume that the following two conditions $(\mathrm{T})$ and $(\mathrm{A})$ are satisfied:

(T) The elasticity tensor $\mathbf{a}(x)$ enjoys the property of symmetry

$$
\mathrm{a}_{i j \ell m}(x)=\mathrm{a}_{\ell m i j}(x)=\mathrm{a}_{j i \ell m}(x), \quad x \in \bar{\Omega},
$$

and is uniformly pointwise stable, that is, there is a constant $\eta>0$ such that

$$
\frac{1}{2} \boldsymbol{e} \cdot \mathbf{a}(x) \cdot \boldsymbol{e} \geq \eta\|\boldsymbol{e}\|^{2}, \quad x \in \bar{\Omega},
$$

for all symmetric two tensors $\boldsymbol{e}$.

(A) $0 \leq \alpha(x) \leq 1$ on $\partial \Omega$, but $\alpha(x) \not \equiv 1$ on $\partial \Omega$.

Then the operator

$$
\left(\boldsymbol{A}, \boldsymbol{B}_{\alpha}\right): \boldsymbol{H}^{s, p}\left(\Omega, \mathbf{R}^{3}\right) \mapsto \boldsymbol{H}^{s-2, p}\left(\Omega, \mathbf{R}^{3}\right) \times \boldsymbol{B}_{(\alpha)}^{s-1-1 / p, p}\left(\partial \Omega, \mathbf{R}^{3}\right)
$$

is an algebraic and topological isomorphism.

It should be noticed that condition (T) is nothing but condition (S) in Main Theorem if we take $\mathbf{a}(x):=\AA \mathbf{A}(X)$.

We give a typical example of a homogeneous, isotropic, elastic material whose reference configuration is a natural state (cf. [MH, Chapter 4, Proposition 3.13]):

EXAMPLE 3.1. We let

$$
\mathrm{a}_{i j \ell m}=\lambda \delta_{i j} \delta_{\ell m}+\mu\left(\delta_{i \ell} \delta_{j m}+\delta_{i m} \delta_{j \ell}\right),
$$

where $\lambda$ and $\mu$ are Lamé moduli, and assume that the following two conditions (L) and (A) are satisfied:

(L) $\mu>0$ and $\lambda+\frac{2}{3} \mu>0$.

(A) $0 \leq \alpha(x) \leq 1$ on $\partial \Omega$, but $\alpha(x) \not \equiv 1$ on $\partial \Omega$.

Then the mixed displacement-traction problem 


$$
\begin{cases}\operatorname{div}(\lambda \operatorname{tr}(\boldsymbol{e}(\boldsymbol{u})) \boldsymbol{I}+2 \mu \boldsymbol{e}(\boldsymbol{u}))=\boldsymbol{f} & \text { in } \Omega, \\ \alpha(x)(\boldsymbol{\tau}(\boldsymbol{u}) \cdot \boldsymbol{n})+(1-\alpha(x)) \boldsymbol{u}=\boldsymbol{\varphi} & \text { on } \partial \boldsymbol{\Omega}\end{cases}
$$

has a unique solution $\boldsymbol{u} \in \boldsymbol{H}^{s, p}\left(\Omega, \mathbf{R}^{3}\right)$ for any $\boldsymbol{f} \in \boldsymbol{H}^{s-2, p}\left(\Omega, \mathbf{R}^{3}\right)$ and any $\boldsymbol{\varphi} \in$ $\boldsymbol{B}_{(\alpha)}^{s-1-1 / p, p}\left(\partial \boldsymbol{\Omega}, \mathbf{R}^{3}\right)$. Here $\boldsymbol{e}(\boldsymbol{u})=\left(e_{i j}(u)\right)$ is the linearized strain tensor defined by the formula

$$
e_{i j}(u):=\frac{1}{2}\left(\frac{\partial u_{i}}{\partial x_{j}}+\frac{\partial u_{j}}{\partial x_{i}}\right),
$$

and $\boldsymbol{\tau}(\boldsymbol{u})=\left(\tau_{i j}(u)\right)$ is the linearized stress tensor defined by the formula

$$
\tau_{i j}(u):=\lambda\left(\sum_{k=1}^{3} e_{k k}(u)\right) \delta_{i j}+2 \mu e_{i j}(u) .
$$

It should be noticed that condition $(\mathrm{L})$ is nothing but condition $(\mathrm{C})$ in Theorem 1.2 .

In order to prove Theorem 3.1, it suffices to show that the operator $\left(\boldsymbol{A}, \boldsymbol{B}_{\alpha}\right)$ is bijective. Indeed, the continuity of the inverse of $\left(\boldsymbol{A}, \boldsymbol{B}_{\alpha}\right)$ follows immediately from an application of Banach's open mapping theorem, since $\left(\boldsymbol{A}, \boldsymbol{B}_{\alpha}\right)$ is a continuous operator.

Theorem 3.1 will be proved in a series of theorems (Theorems 5.1, 6.1 and 7.1) in the subsequent chapters.

\section{Reduction to the Boundary}

In Chapters 4 through 7 we study the linearized problem (1.4) in the framework of Sobolev spaces of $L^{p}$ type, by using the $L^{p}$ theory of pseudodifferential operators. In this chapter we show that problem (1.4) can be reduced to the study of a $3 \times 3$ matrix-valued, pseudo-differential operator on the boundary.

\subsection{Operator $T_{\alpha}$}

First, we consider the pure displacement problem (Dirichlet problem)

$$
\begin{cases}\operatorname{div}(\mathbf{a}(x) \cdot \nabla \boldsymbol{v})=\boldsymbol{f} & \text { in } \Omega, \\ \boldsymbol{v}=\boldsymbol{\varphi} & \text { on } \partial \Omega .\end{cases}
$$

We let 


$$
\begin{aligned}
& \boldsymbol{A} \boldsymbol{v}=\operatorname{div}(\mathbf{a}(x) \cdot \nabla \boldsymbol{v}), \\
& \boldsymbol{\gamma} \boldsymbol{v}=\left.\boldsymbol{v}\right|_{\partial \Omega},
\end{aligned}
$$

and associate with problem (D) a linear operator

$$
(\boldsymbol{A}, \boldsymbol{\gamma}): \boldsymbol{H}^{s, p}\left(\boldsymbol{\Omega}, \mathbf{R}^{3}\right) \mapsto \boldsymbol{H}^{s-2, p}\left(\boldsymbol{\Omega}, \mathbf{R}^{3}\right) \times \boldsymbol{B}^{s-1 / p, p}\left(\partial \Omega, \mathbf{R}^{3}\right) .
$$

Then we have the following existence and uniqueness theorem for problem (D) (cf. [MH, Chapter 6, Theorem 1.11]; [It1, Lemma 1.3]):

THEOREM 4.1. Let $1<p<\infty$. If condition $(\mathrm{T})$ is satisfied, then the operator

$$
(\boldsymbol{A}, \boldsymbol{\gamma}): \boldsymbol{H}^{s, p}\left(\boldsymbol{\Omega}, \mathbf{R}^{3}\right) \rightarrow \boldsymbol{H}^{s-2, p}\left(\boldsymbol{\Omega}, \mathbf{R}^{3}\right) \times \boldsymbol{B}^{s-1 / p, p}\left(\partial \boldsymbol{\Omega}, \mathbf{R}^{3}\right)
$$

is an algebraic and topological isomorphism, for all $s>1 / p$.

By Theorem 4.1, we can introduce a linear operator

$$
\mathscr{P}: \boldsymbol{B}^{s-1 / p, p}\left(\partial \Omega, \mathbf{R}^{3}\right) \rightarrow \boldsymbol{H}^{s, p}\left(\Omega, \mathbf{R}^{3}\right)
$$

as follows: For any $\boldsymbol{\varphi} \in \boldsymbol{B}^{s-1 / p, p}\left(\partial \Omega, \mathbf{R}^{3}\right)$, the function $\mathscr{P} \boldsymbol{\varphi}$ is the unique solution of the pure displacement problem

$$
\begin{cases}A \boldsymbol{v}=\mathbf{0} & \text { in } \Omega, \\ \boldsymbol{v}=\boldsymbol{\varphi} & \text { on } \partial \Omega .\end{cases}
$$

The operator $\mathscr{P}$ is called the Poisson operator for problem (D).

It should be noticed that the spaces

$$
\boldsymbol{N}(\boldsymbol{A}, s, p)=\left\{\boldsymbol{w} \in \boldsymbol{H}^{s, p}\left(\Omega, \mathbf{R}^{3}\right): \boldsymbol{A w}=\mathbf{0} \text { in } \Omega\right\}
$$

and

$$
\boldsymbol{B}^{s-1 / p, p}\left(\partial \Omega, \mathbf{R}^{3}\right)
$$

are isomorphic in such a way that

$$
\begin{aligned}
& \boldsymbol{N}(\boldsymbol{A}, s, p) \stackrel{\stackrel{\gamma}{\rightarrow}}{B^{s-1 / p, p}}\left(\partial \boldsymbol{\Omega}, \mathbf{R}^{3}\right) \\
& \boldsymbol{N}(\boldsymbol{A}, s, p) \underset{\mathscr{P}}{\leftarrow} \boldsymbol{B}^{s-1 / p, p}\left(\partial \Omega, \mathbf{R}^{3}\right)
\end{aligned}
$$

Secondly, we consider the following non-degenerate mixed displacementtraction problem: 


$$
\begin{cases}\operatorname{div}(\mathbf{a}(x) \cdot \nabla \boldsymbol{v})=\boldsymbol{f} & \text { in } \Omega \\ (\mathbf{a}(x) \cdot \nabla \boldsymbol{v} \cdot \boldsymbol{n})+\boldsymbol{v}=\boldsymbol{\varphi} & \text { on } \partial \Omega\end{cases}
$$

We let

$$
\begin{aligned}
& \boldsymbol{A} \boldsymbol{v}=\operatorname{div}(\mathbf{a}(x) \cdot \nabla \boldsymbol{v}), \\
& \boldsymbol{B} \boldsymbol{v}=\left.(\mathbf{a}(x) \cdot \boldsymbol{\nabla} \boldsymbol{v} \cdot \boldsymbol{n})\right|_{\partial \boldsymbol{\Omega}},
\end{aligned}
$$

and associate with problem $(\mathrm{M})$ a linear operator

$$
(\boldsymbol{A}, \boldsymbol{B}+\boldsymbol{\gamma}): \boldsymbol{H}^{s, p}\left(\Omega, \mathbf{R}^{3}\right) \mapsto \boldsymbol{H}^{s-2, p}\left(\Omega, \mathbf{R}^{3}\right) \times \boldsymbol{B}^{s-1-1 / p, p}\left(\partial \Omega, \mathbf{R}^{3}\right) .
$$

Then we have the following existence and uniqueness theorem for problem (M) (cf. [MH, Chapter 6, Theorem 1.11]; [It1, Lemma 1.3]):

THEOREM 4.2. Let $1<p<\infty$. If condition $(\mathrm{T})$ is satisfied, then the operator

$$
(\boldsymbol{A}, \boldsymbol{B}+\boldsymbol{\gamma}): \boldsymbol{H}^{s, p}\left(\boldsymbol{\Omega}, \mathbf{R}^{3}\right) \mapsto \boldsymbol{H}^{s-2, p}\left(\Omega, \mathbf{R}^{3}\right) \times \boldsymbol{B}^{s-1-1 / p, p}\left(\partial \Omega, \mathbf{R}^{3}\right)
$$

is an algebraic and topological isomorphism, for all $s>1 / p+1$.

Now, by making use of problems (D) and (M) we show that problem (1.4) can be reduced to the study of a $3 \times 3$ matrix-valued, pseudo-differential operator on the boundary.

Let $\boldsymbol{f}$ be an arbitrary element of $\boldsymbol{H}^{s-2, p}\left(\Omega, \mathbf{R}^{3}\right)$, and $\boldsymbol{\varphi}$ an arbitrary element of $\boldsymbol{B}_{(\alpha)}^{s-1-1 / p, p}\left(\partial \Omega, \mathbf{R}^{3}\right)$ such that

$$
\boldsymbol{\varphi}=\alpha(x) \boldsymbol{\varphi}_{1}+(1-\alpha(x)) \boldsymbol{\varphi}_{2}
$$

with

$$
\boldsymbol{\varphi}_{1} \in \boldsymbol{B}^{s-1-1 / p, p}\left(\partial \Omega, \mathbf{R}^{3}\right), \quad \boldsymbol{\varphi}_{2} \in \boldsymbol{B}^{s-1 / p, p}\left(\partial \Omega, \mathbf{R}^{3}\right) .
$$

We assume that $\boldsymbol{u} \in \boldsymbol{H}^{s, p}\left(\Omega, \mathbf{R}^{3}\right)$ is a solution of the mixed displacement-traction problem

$$
\begin{cases}\boldsymbol{A} \boldsymbol{u}=\boldsymbol{f} & \text { in } \Omega, \\ \boldsymbol{B}_{\alpha} \boldsymbol{u}=\alpha(x) \boldsymbol{B} \boldsymbol{u}+(1-\alpha(x)) \gamma \boldsymbol{u}=\boldsymbol{\varphi} & \text { on } \partial \Omega .\end{cases}
$$

By Theorem 4.2, we can find an element $\boldsymbol{v} \in \boldsymbol{H}^{s, p}\left(\Omega, \mathbf{R}^{3}\right)$ such that

$$
\begin{cases}A v=f & \text { in } \Omega, \\ B v+\gamma v=\varphi_{1}-\varphi_{2} & \text { on } \partial \Omega .\end{cases}
$$

We let 


$$
\boldsymbol{w}=\boldsymbol{u}-\boldsymbol{v}
$$

Then it is easy to see that $\boldsymbol{w} \in \boldsymbol{H}^{s, p}\left(\Omega, \mathbf{R}^{3}\right)$ is a solution of the mixed displacement-traction problem

$$
\begin{cases}\boldsymbol{A} \boldsymbol{w}=\mathbf{0} & \text { in } \Omega, \\ \boldsymbol{B}_{\alpha} \boldsymbol{w}=\boldsymbol{\varphi}_{2}+(2 \alpha(x)-1) \boldsymbol{\gamma} \boldsymbol{v} & \text { on } \partial \Omega .\end{cases}
$$

However, the Poisson operator $\mathscr{P}$ is an isomorphism of $\boldsymbol{B}^{s-1 / p, p}\left(\partial \Omega, \mathbf{R}^{3}\right)$ onto the null space $\boldsymbol{N}(\boldsymbol{A}, s, p)$. Therefore, we find that $\boldsymbol{w} \in \boldsymbol{H}^{s, p}\left(\Omega, \mathbf{R}^{3}\right)$ is a solution of problem (4.1) if and only if $\boldsymbol{\psi} \in \boldsymbol{B}^{s-1 / p, p}\left(\partial \Omega, \mathbf{R}^{3}\right)$ is a solution of the equation

$$
\boldsymbol{B}_{\alpha}\left(\mathscr{P}_{\boldsymbol{\psi}}\right)=\boldsymbol{\varphi}_{2}+(2 \alpha(x)-1) \boldsymbol{\gamma} \boldsymbol{v} \text { on } \partial \Omega .
$$

Here $\boldsymbol{\psi}=\boldsymbol{\gamma} \boldsymbol{w}$, or equivalently, $\boldsymbol{w}=\mathscr{P} \boldsymbol{\psi}$. This is a generalization of the classical Fredholm integral equation.

Summing up, we have proved the following:

Proposition 4.3. Let $1<p<\infty$ and $s>1 / p+1$. Then problem (1.4) has a solution $\boldsymbol{u} \in \boldsymbol{H}^{s, p}\left(\boldsymbol{\Omega}, \mathbf{R}^{3}\right)$ for $\boldsymbol{f} \in \boldsymbol{H}^{s-2, p}\left(\Omega, \mathbf{R}^{3}\right)$ and $\boldsymbol{\varphi} \in \boldsymbol{B}_{(\alpha)}^{s-1-1 / p, p}\left(\partial \boldsymbol{\Omega}, \mathbf{R}^{3}\right)$ if and only if equation (4.2) has a solution $\boldsymbol{\psi} \in \boldsymbol{B}^{s-1 / p, p}\left(\partial \Omega, \mathbf{R}^{3}\right)$.

Now we let

$$
\begin{gathered}
\boldsymbol{T}_{\alpha}: \boldsymbol{C}^{\infty}\left(\partial \boldsymbol{\Omega}, \mathbf{R}^{3}\right) \rightarrow \boldsymbol{C}^{\infty}\left(\partial \Omega, \mathbf{R}^{3}\right) \\
\boldsymbol{\varphi} \mapsto \boldsymbol{B}_{\alpha}(\mathscr{P} \boldsymbol{\varphi}) .
\end{gathered}
$$

Then we have the formula

$$
\boldsymbol{T}_{\alpha}=\alpha(x) \boldsymbol{\Pi}+(1-\alpha(x)) \boldsymbol{I},
$$

where

$$
\Pi \boldsymbol{\varphi}=\boldsymbol{B}(\mathscr{P} \boldsymbol{\varphi})=\left.\mathbf{a}(x) \cdot \nabla(\mathscr{P} \boldsymbol{\varphi}) \cdot \boldsymbol{n}\right|_{\partial \Omega} .
$$

It is known (cf. [Hol], [Se]) that the operator $\boldsymbol{\Pi}$ is a $3 \times 3$ matrix-valued, classical pseudo-differential operator of first order on the boundary $\partial \Omega$; hence the operator $\boldsymbol{T}_{\alpha}$ is a $3 \times 3$ matrix-valued, classical pseudo-differential operator of first order on the boundary $\partial \Omega$.

Consequently, Proposition 4.3 asserts that problem (1.4) can be reduced to the study of the system $\boldsymbol{T}_{\alpha}$ of pseudo-differential operators on the boundary $\partial \Omega$. We shall formulate this fact more precisely in terms of functional analysis.

We associate with problem (1.4) a continuous linear operator 


$$
\mathscr{A}_{\alpha}=\left(\boldsymbol{A}, \boldsymbol{B}_{\alpha}\right): \boldsymbol{H}^{s, p}\left(\Omega, \mathbf{R}^{3}\right) \rightarrow \boldsymbol{H}^{s-2, p}\left(\Omega, \mathbf{R}^{3}\right) \times \boldsymbol{B}_{(\alpha)}^{s-1-1 / p, p}\left(\partial \Omega, \mathbf{R}^{3}\right) .
$$

Similarly, we associate with equation (4.2) a linear operator

$$
\mathscr{T}_{\alpha}: \boldsymbol{B}^{s-1 / p, p}\left(\partial \Omega, \mathbf{R}^{3}\right) \rightarrow \boldsymbol{B}^{s-1 / p, p}\left(\partial \Omega, \mathbf{R}^{3}\right)
$$

as follows.

(a) The domain $D\left(\mathscr{T}_{\alpha}\right)$ of $\mathscr{T}_{\alpha}$ is the space

$$
D\left(\mathscr{T}_{\alpha}\right)=\left\{\boldsymbol{\varphi} \in \boldsymbol{B}^{s-1 / p, p}\left(\partial \Omega, \mathbf{R}^{3}\right): \boldsymbol{T}_{\alpha} \boldsymbol{\varphi} \in B^{s-1 / p, p}\left(\partial \boldsymbol{\Omega}, \mathbf{R}^{3}\right)\right\} .
$$

(b) $\mathscr{T}_{\alpha} \varphi=\boldsymbol{T}_{\alpha} \boldsymbol{\varphi}, \boldsymbol{\varphi} \in D\left(\mathscr{T}_{\alpha}\right)$.

It should be noticed that the operator $\mathscr{T}_{\alpha}$ is a densely defined, closed linear operator, since the operator $\boldsymbol{T}_{\alpha}: \boldsymbol{B}^{s-1 / p, p}\left(\partial \Omega, \mathbf{R}^{3}\right) \rightarrow \boldsymbol{B}^{s-1-1 / p, p}\left(\partial \Omega, \mathbf{R}^{3}\right)$ is continuous and since the domain $D\left(\mathscr{T}_{\alpha}\right)$ contains the space $\boldsymbol{C}^{\infty}\left(\partial \Omega, \mathbf{R}^{3}\right)$.

Then Proposition 4.3 can be reformulated in the following form (cf. [Ta1, Section 8.3]):

THEOREM 4.4. (i) The null space $\mathbf{N}\left(\mathscr{A}_{\alpha}\right)$ of $\mathscr{A}_{\alpha}$ has finite dimension if and only if the null space $N\left(\mathscr{T}_{\alpha}\right)$ of $\mathscr{T}_{\alpha}$ has finite dimension, and we have the formula

$$
\operatorname{dim} N\left(\mathscr{A}_{\alpha}\right)=\operatorname{dim} N\left(\mathscr{T}_{\alpha}\right) .
$$

(ii) The range $\boldsymbol{R}\left(\mathscr{A}_{\alpha}\right)$ of $\mathscr{A}_{\alpha}$ is closed if and only if the range $\boldsymbol{R}\left(\mathscr{T}_{\alpha}\right)$ of $\mathscr{T}_{\alpha}$ is closed; and $\boldsymbol{R}\left(\mathscr{A}_{\alpha}\right)$ has finite codimension if and only if $\boldsymbol{R}\left(\mathscr{T}_{\alpha}\right)$ has finite codimension, and we have the formula

$$
\operatorname{codim} \boldsymbol{R}\left(\mathscr{A}_{\alpha}\right)=\operatorname{codim} \boldsymbol{R}\left(\mathscr{T}_{\alpha}\right) .
$$

(iii) The operator $\mathscr{A}_{\alpha}$ is a Fredholm operator if and only if the operator $\mathscr{T}_{\alpha}$ is a Fredholm operator, and we have the formula

$$
\text { ind } \mathscr{A}_{\alpha}=\text { ind } \mathscr{T}_{\alpha} \text {. }
$$

Here we recall that a densely defined, closed linear operator $T$ from a Banach space $X$ into a Banach space $Y$ is called a Fredholm operator if it satisfies the following three conditions (a), (b) and (c):

(a) The null space $N(T)=\{x \in D(T): T x=0\}$ of $T$ has finite dimension; $\operatorname{dim} N(T)<\infty$.

(b) The range $R(T)=\{T x: x \in D(T)\}$ of $T$ is closed in $Y$.

(c) The range $R(T)$ has finite codimension in $Y ; \quad \operatorname{codim} R(T)=$ $\operatorname{dim} Y / R(T)<\infty$.

In this case, the index of $T$ is defined by the formula 


$$
\text { ind } T:=\operatorname{dim} N(T)-\operatorname{codim} R(T) \text {. }
$$

Furthermore, the next theorem states that the operator $\mathscr{A}_{\alpha}$ has regularity property if and only if the operator $\mathscr{T}_{\alpha}$ has.

THEOREM 4.5. Let $1<p<\infty$ and $s>1 / p+1$. Then the following two conditions (i) and (ii) are equivalent:

$$
\begin{gathered}
\boldsymbol{u} \in \boldsymbol{L}^{p}\left(\Omega, \mathbf{R}^{3}\right), \quad \boldsymbol{A} \boldsymbol{u} \in \boldsymbol{H}^{s-2, p}\left(\Omega, \mathbf{R}^{3}\right), \\
\boldsymbol{B}_{\alpha} \boldsymbol{u} \in \boldsymbol{B}_{(\alpha)}^{s-1-1 / p, p}\left(\partial \Omega, \mathbf{R}^{3}\right) \Rightarrow \boldsymbol{u} \in \boldsymbol{H}^{s, p}\left(\Omega, \mathbf{R}^{3}\right) . \\
\boldsymbol{\varphi} \in \boldsymbol{B}^{-1 / p, p}\left(\partial \Omega, \mathbf{R}^{3}\right), \quad \boldsymbol{T}_{\alpha} \boldsymbol{\varphi} \in \boldsymbol{B}^{s-1 / p, p}\left(\partial \Omega, \mathbf{R}^{3}\right) \Rightarrow \boldsymbol{\varphi} \in \boldsymbol{B}^{s-1 / p, p}\left(\partial \Omega, \mathbf{R}^{3}\right) .
\end{gathered}
$$

Proof. (i) $\Rightarrow$ (ii): First, just as in [Ta1, Proposition 8.3.2] we can prove that the boundary condition $\boldsymbol{B}_{\alpha} \boldsymbol{u}$ is defined as an function in $\boldsymbol{B}^{-1-1 / p, p}\left(\partial \boldsymbol{\Omega}, \mathbf{R}^{3}\right)$ if $\boldsymbol{u} \in \boldsymbol{L}^{p}\left(\Omega, \mathbf{R}^{3}\right)$ and $\boldsymbol{A} \boldsymbol{u} \in \boldsymbol{H}^{s-2, p}\left(\Omega, \mathbf{R}^{3}\right)$. Furthermore, we remark that the Poisson operator $\mathscr{P}$ is an isomorphism of $\boldsymbol{B}^{t-1 / p, p}\left(\partial \Omega, \mathbf{R}^{3}\right)$ onto the null space $\boldsymbol{N}(\boldsymbol{A}, t, p)=$ $\left\{\boldsymbol{w} \in \boldsymbol{H}^{t, p}\left(\Omega, \mathbf{R}^{3}\right): \boldsymbol{A w}=\mathbf{0}\right.$ in $\left.\Omega\right\}$ for all $t \in \mathbf{R}$.

Now we assume that

$$
\boldsymbol{\varphi} \in \boldsymbol{B}^{-1 / p, p}\left(\partial \Omega, \mathbf{R}^{3}\right) \quad \text { and } \quad \boldsymbol{T}_{\alpha} \boldsymbol{\varphi} \in \boldsymbol{B}^{s-1 / p, p}\left(\partial \Omega, \mathbf{R}^{3}\right) .
$$

Then, by letting $\boldsymbol{u}=\mathscr{P} \boldsymbol{\varphi}$ we obtain that

$$
\boldsymbol{u} \in L^{p}\left(\boldsymbol{\Omega}, \mathbf{R}^{3}\right), \quad \boldsymbol{A} \boldsymbol{u}=\mathbf{0} \quad \text { and } \quad \boldsymbol{B}_{\alpha} \boldsymbol{u}=\boldsymbol{T}_{\alpha} \boldsymbol{\varphi} \in \boldsymbol{B}^{s-1 / p, p}\left(\partial \Omega, \mathbf{R}^{3}\right) .
$$

Hence it follows from condition (i) that

$$
\boldsymbol{u} \in \boldsymbol{H}^{s, p}\left(\Omega, \mathbf{R}^{3}\right),
$$

so that, by Theorem 4.1,

$$
\boldsymbol{\varphi}=\boldsymbol{\gamma} \boldsymbol{u} \in \boldsymbol{B}^{s-1 / p, p}\left(\partial \Omega, \mathbf{R}^{3}\right) .
$$

(ii) $\Rightarrow$ (i): Conversely, we assume that

$$
\boldsymbol{u} \in \boldsymbol{L}^{p}\left(\boldsymbol{\Omega}, \mathbf{R}^{3}\right), \quad \boldsymbol{A} \boldsymbol{u} \in \boldsymbol{H}^{s-2, p}\left(\Omega, \mathbf{R}^{3}\right) \quad \text { and } \quad \boldsymbol{B}_{\alpha} \boldsymbol{u} \in \boldsymbol{B}_{(\alpha)}^{s-1-1 / p, p}\left(\partial \Omega, \mathbf{R}^{3}\right),
$$

where

$$
\boldsymbol{B}_{\alpha} \boldsymbol{u}=\alpha(x) \boldsymbol{\varphi}_{1}+(1-\alpha(x)) \boldsymbol{\varphi}_{2},
$$

with

$$
\boldsymbol{\varphi}_{1} \in \boldsymbol{B}^{s-1-1 / p, p}\left(\partial \Omega, \mathbf{R}^{3}\right), \quad \boldsymbol{\varphi}_{2} \in \boldsymbol{B}^{s-1 / p, p}\left(\partial \Omega, \mathbf{R}^{3}\right) .
$$


Then the function $\boldsymbol{u}$ can be decomposed as follows:

$$
\boldsymbol{u}=\boldsymbol{v}+\boldsymbol{w},
$$

where $\boldsymbol{v} \in \boldsymbol{H}^{s, p}\left(\Omega, \mathbf{R}^{3}\right)$ is a unique solution of the non-degenerate mixed displacement-traction problem

$$
\begin{cases}A v=A u & \text { in } \Omega, \\ B v+\gamma v=\varphi_{1}-\varphi_{2} & \text { in } \partial \Omega\end{cases}
$$

and so

$$
\boldsymbol{w}=\boldsymbol{u}-\boldsymbol{v} \in \boldsymbol{N}(\boldsymbol{A}, 0, p) .
$$

Theorem 4.1 asserts that the function $\boldsymbol{w}$ can be written as follows:

$$
\boldsymbol{w}=\mathscr{P} \boldsymbol{\varphi}, \quad \boldsymbol{\varphi}=\boldsymbol{\gamma} \boldsymbol{w} \in \boldsymbol{B}^{-1 / p, p}\left(\partial \boldsymbol{\Omega}, \mathbf{R}^{3}\right) .
$$

Hence we have the formula

$$
\boldsymbol{T}_{\alpha} \boldsymbol{\varphi}=\boldsymbol{B}_{\alpha} \boldsymbol{w}=\boldsymbol{B}_{\alpha} \boldsymbol{u}-\boldsymbol{B}_{\alpha} \boldsymbol{v}=\boldsymbol{\varphi}_{2}+(2 \alpha(x)-1) \boldsymbol{\gamma} \boldsymbol{v} \in \boldsymbol{B}^{s-1 / p, p}\left(\partial \Omega, \mathbf{R}^{3}\right) .
$$

Thus it follows from condition (ii) that

$$
\boldsymbol{\varphi} \in \boldsymbol{B}^{s-1 / p, p}\left(\partial \Omega, \mathbf{R}^{3}\right),
$$

so that, again by Theorem 4.1,

$$
\boldsymbol{w}=\mathscr{P} \boldsymbol{\varphi} \in \boldsymbol{H}^{s, p}\left(\Omega, \mathbf{R}^{3}\right) .
$$

This proves that

$$
\boldsymbol{u}=\boldsymbol{v}+\boldsymbol{w} \in \boldsymbol{H}^{s, p}\left(\Omega, \mathbf{R}^{3}\right) .
$$

The proof of Theorem 4.5 is complete.

\subsection{Operator $\Pi$}

We recall that the operator $\boldsymbol{T}_{\alpha}$, defined by the formula

$$
\begin{gathered}
\boldsymbol{T}_{\alpha}: \boldsymbol{C}^{\infty}\left(\partial \boldsymbol{\Omega}, \mathbf{R}^{3}\right) \rightarrow \boldsymbol{C}^{\infty}\left(\partial \Omega, \mathbf{R}^{3}\right) \\
\boldsymbol{\varphi} \mapsto \boldsymbol{B}_{\alpha}(\mathscr{P} \boldsymbol{\varphi})
\end{gathered}
$$

can be written as follows:

$$
\boldsymbol{T}_{\alpha}=\alpha(x) \boldsymbol{\Pi}+(1-\alpha(x)) \boldsymbol{I},
$$

where 


$$
\Pi \boldsymbol{\varphi}=\boldsymbol{B}(\mathscr{P} \boldsymbol{\varphi})=\left.\mathbf{a}(x) \cdot \nabla(\mathscr{P} \boldsymbol{\varphi}) \cdot \boldsymbol{n}\right|_{\partial \Omega} .
$$

In this section we prove some properties of the operator $\boldsymbol{\Pi}$ as a $3 \times 3$ matrixvalued, pseudo-differential operator. To do this, we need the following Green's formula and Korn's inequalities:

TheOREM 4.6 (Green's formula). We have, for all $\boldsymbol{u}, \boldsymbol{v} \in \boldsymbol{C}^{\infty}\left(\bar{\Omega}, \mathbf{R}^{3}\right)$,

$$
\int_{\Omega} \boldsymbol{u} \cdot \operatorname{div}(\mathbf{a}(x) \cdot \nabla \boldsymbol{v}) d x=\int_{\partial \Omega} \boldsymbol{u}[\mathbf{a}(x) \cdot \nabla \boldsymbol{v} \cdot \boldsymbol{n}] d a-\int_{\Omega} \boldsymbol{\nabla} \boldsymbol{u} \cdot \mathbf{a}(x) \cdot \nabla \boldsymbol{v} d x
$$

Here $d a$ is the area element on the boundary $\partial \Omega$.

By the symmetry of the tensor a(x), Green's formula (4.3) follows from an application of the divergence theorem.

We recall that the linearized strain tensor $\boldsymbol{e}(\boldsymbol{u})=\left(e_{i j}(u)\right)$ is defined by the formula

$$
e_{i j}(u):=\frac{1}{2}\left(\frac{\partial u_{i}}{\partial x_{j}}+\frac{\partial u_{j}}{\partial x_{i}}\right)
$$

The next inequalities are special cases of Gårding's inequality for the elliptic operator $\boldsymbol{u} \mapsto \boldsymbol{e}(\boldsymbol{u})$ (cf. [DL, Chapitre 3, Théorèmes 3.1 et 3.3]):

THEOREM 4.7 (Korn's inequalities). (i) For every non-empty open subset $\gamma \subset \partial \Omega$, there exists a constant $c(\gamma)>0$ such that

$$
\int_{\Omega}\|\boldsymbol{e}(\boldsymbol{u})\|^{2} d x \geq c(\gamma)\left(\int_{\Omega}\|\boldsymbol{u}\|^{2} d x+\int_{\Omega}\|\nabla \boldsymbol{u}\|^{2} d x\right)
$$

for all $\boldsymbol{u} \in \boldsymbol{H}^{1,2}\left(\Omega, \mathbf{R}^{3}\right)$ satisfying the condition $\boldsymbol{u}=\mathbf{0}$ on $\gamma$.

(ii) There exists a constant $c>0$ such that

$$
\int_{\Omega}\|\boldsymbol{e}(\boldsymbol{u})\|^{2} d x+\int_{\Omega}\|\boldsymbol{u}\|^{2} d x \geq c\left(\int_{\Omega}\|\boldsymbol{u}\|^{2} d x+\int_{\Omega}\|\boldsymbol{\nabla} \boldsymbol{u}\|^{2} d x\right)
$$

for all $\boldsymbol{u} \in \boldsymbol{H}^{1,2}\left(\Omega, \mathbf{R}^{3}\right)$.

Now we can prove the following (cf. [It1, Proposition 1.4]):

THEOREM 4.8. (i) The operator $\boldsymbol{\Pi}$ is formally self-adjoint: $\boldsymbol{\Pi}^{*}=\boldsymbol{\Pi}$. 
(ii) The operator $\boldsymbol{\Pi}$ is strongly elliptic, that is, there exist constants $c_{1}>0$ and $c_{2}>0$ such that we have, for all $\boldsymbol{\varphi} \in \boldsymbol{C}^{\infty}\left(\partial \Omega, \mathbf{C}^{3}\right)$,

$$
\int_{\partial \Omega} \Pi \boldsymbol{\varphi} \cdot \overline{\boldsymbol{\varphi}} d a \geq c_{1}|\boldsymbol{\varphi}|_{1 / 2,2}^{2}-c_{2}|\boldsymbol{\varphi}|_{-1 / 2,2}^{2}
$$

Here $\overline{\boldsymbol{\varphi}}$ denotes the complex conjugate of $\boldsymbol{\varphi}$.

(iii) The principal symbol $\boldsymbol{p}_{1}\left(x^{\prime}, \xi^{\prime}\right)$ of $\boldsymbol{\Pi}$ satisfies the condition

$$
\boldsymbol{p}_{1}\left(x^{\prime}, \xi^{\prime}\right) \geq c_{0}\left|\xi^{\prime}\right| \boldsymbol{I} \quad \text { on } T^{*}(\partial \Omega),
$$

with a constant $c_{0}>0$. Here $T^{*}(\partial \Omega)$ is the cotangent bundle of $\partial \Omega$ and $\left|\xi^{\prime}\right|$ is the length of $\xi^{\prime}$ with respect to the Riemannian metric of $\partial \Omega$ induced by the natural metric of $\mathbf{R}^{3}$.

Proof. (i) If $\boldsymbol{\varphi}$ and $\boldsymbol{\psi}$ are functions in $\boldsymbol{C}^{\infty}\left(\partial \Omega, \mathbf{C}^{3}\right)$, then, by applying Green's formula (4.3) with $\boldsymbol{u}:=\mathscr{P} \boldsymbol{\varphi}$ and $\boldsymbol{v}:=\mathscr{P} \boldsymbol{\psi}$ we obtain that

$$
\begin{aligned}
0 & =\int_{\partial \Omega} \boldsymbol{\varphi} \cdot[\mathbf{a}(x) \cdot \overline{\nabla(\mathscr{P} \boldsymbol{\psi})} \cdot \boldsymbol{n}] d a-\int_{\Omega} \boldsymbol{\nabla}(\mathscr{P} \boldsymbol{\varphi}) \cdot \mathbf{a}(x) \cdot \overline{\nabla(\mathscr{P} \boldsymbol{\psi})} d x \\
& =\int_{\partial \Omega} \boldsymbol{\varphi} \cdot \overline{\boldsymbol{\Pi} \boldsymbol{\psi}} d a-\int_{\Omega} \boldsymbol{\nabla}(\mathscr{P} \boldsymbol{\varphi}) \cdot \mathbf{a}(x) \cdot \overline{\nabla(\mathscr{P} \boldsymbol{\psi})} d x
\end{aligned}
$$

or equivalently,

$$
\int_{\partial \Omega} \boldsymbol{\Pi} \boldsymbol{\varphi} \cdot \overline{\boldsymbol{\psi}} d a=\int_{\Omega} \overline{\nabla(\mathscr{P} \boldsymbol{\psi})} \cdot \mathbf{a}(x) \cdot \boldsymbol{\nabla}(\mathscr{P} \boldsymbol{\varphi}) d x .
$$

Therefore, by the symmetry of the tensor $\mathbf{a}(x)$ it follows that

$$
\begin{aligned}
\int_{\partial \Omega} \boldsymbol{\Pi} \boldsymbol{\varphi} \cdot \overline{\boldsymbol{\psi}} d a & =\int_{\Omega} \overline{\boldsymbol{\nabla}(\mathscr{P} \boldsymbol{\psi})} \cdot \mathbf{a}(x) \cdot \boldsymbol{\nabla}(\mathscr{P} \boldsymbol{\varphi}) d x \\
& =\int_{\Omega} \boldsymbol{\nabla}(\mathscr{P} \boldsymbol{\varphi}) \cdot \mathbf{a}(x) \cdot \overline{\nabla(\mathscr{P} \boldsymbol{\psi})} d x \\
& =\int_{\partial \Omega} \boldsymbol{\varphi} \cdot \overline{\boldsymbol{\Pi} \boldsymbol{\psi}} d a .
\end{aligned}
$$

This proves the formal self-adjointness of the operator $\boldsymbol{\Pi}$.

(ii) Since the tensor $\mathbf{a}(x)$ is uniformly pointwise stable, it follows from an application of the second Korn inequality (4.5) that we have, for all $\boldsymbol{u} \in \boldsymbol{H}^{1,2}\left(\Omega, \mathbf{C}^{3}\right)$, 


$$
\begin{aligned}
\int_{\Omega} \boldsymbol{\nabla} \boldsymbol{u} \cdot \mathbf{a}(x) \cdot \overline{\nabla \boldsymbol{u}} d x & =\int_{\Omega} \boldsymbol{e}(\boldsymbol{u}) \cdot \mathbf{a}(x) \cdot \overline{\boldsymbol{e}(\boldsymbol{u})} d x \\
& \geq 2 \eta \int_{\Omega}\|\boldsymbol{e}(\boldsymbol{u})\|^{2} d x \\
& \geq 2 \eta c\|\boldsymbol{u}\|_{1,2}^{2}-2 \eta\|\boldsymbol{u}\|_{0,2}^{2} .
\end{aligned}
$$

In particular, by taking $\boldsymbol{u}=\mathscr{P} \boldsymbol{\varphi}$ we have, with $C_{1}:=2 \eta c$ and $C_{2}:=2 \eta$,

$$
\int_{\Omega} \boldsymbol{\nabla}(\mathscr{P} \boldsymbol{\varphi}) \cdot \mathbf{a}(x) \cdot \overline{\nabla(\mathscr{P} \boldsymbol{\varphi})} d x \geq C_{1}\|\mathscr{P} \boldsymbol{\varphi}\|_{1,2}^{2}-C_{2}\|\mathscr{P} \boldsymbol{\varphi}\|_{0,2}^{2}
$$

Hence, by combining this inequality and equality (4.8) with $\boldsymbol{\varphi}:=\boldsymbol{\psi}$ we obtain that

$$
\int_{\partial \Omega} \Pi \boldsymbol{\varphi} \cdot \overline{\boldsymbol{\varphi}} d a \geq C_{1}\|\mathscr{P} \boldsymbol{\varphi}\|_{1,2}^{2}-C_{2}\|\mathscr{P} \boldsymbol{\varphi}\|_{0,2}^{2}
$$

However, we recall that the Poisson operator $\mathscr{P}$ is an isomorphism of the space

$$
\boldsymbol{B}^{s-1 / 2,2}\left(\partial \Omega, \mathbf{R}^{3}\right)
$$

onto the null space

$$
\boldsymbol{N}(\boldsymbol{A}, s, 2)=\left\{\boldsymbol{w} \in \boldsymbol{H}^{s, 2}\left(\Omega, \mathbf{R}^{3}\right): \boldsymbol{A} \boldsymbol{w}=\mathbf{0} \text { in } \Omega\right\}
$$

for all $s \in \mathbf{R}$.

Therefore, the desired inequality (4.6) follows from inequality (4.9).

(iii) It is known (cf. [Ho3], [Ku], [Ty]) that inequality (4.6) implies the strong ellipticity (4.7) of the operator $\boldsymbol{\Pi}$.

The proof of Theorem 4.8 is complete.

\section{Regularity Theorem for Problem (1.4)}

In this chapter we prove the following regularity theorem for problem (1.4):

THEOREM 5.1. Let $1<p<\infty$. If condition $(\mathrm{T})$ is satisfied, then we have, for any $s>1 / p+1$,

$$
\begin{gathered}
\boldsymbol{u} \in \boldsymbol{L}^{p}\left(\Omega, \mathbf{R}^{3}\right), \quad \boldsymbol{A} \boldsymbol{u} \in \boldsymbol{H}^{s-2, p}\left(\Omega, \mathbf{R}^{3}\right), \\
\boldsymbol{B}_{\alpha} \boldsymbol{u} \in \boldsymbol{B}_{(\alpha)}^{s-1-1 / p, p}\left(\partial \Omega, \mathbf{R}^{3}\right) \Rightarrow \boldsymbol{u} \in \boldsymbol{H}^{s, p}\left(\Omega, \mathbf{R}^{3}\right) .
\end{gathered}
$$

Proof. By Theorem 4.5, we are reduced to the study of a $3 \times 3$ matrixvalued, pseudo-differential operator 


$$
\boldsymbol{T}_{\alpha}=\alpha(x) \boldsymbol{\Pi}+(1-\alpha(x)) \boldsymbol{I}
$$

on the boundary $\partial \Omega$. Therefore, it suffices to prove the following:

Lemma 5.2. If condition ( $\mathrm{T}$ ) is satisfied, then we have, for all $s \in \mathbf{R}$,

$$
\boldsymbol{\varphi} \in \mathscr{D}^{\prime}\left(\partial \Omega, \mathbf{R}^{3}\right), \quad \boldsymbol{T}_{\alpha} \boldsymbol{\varphi} \in \boldsymbol{B}^{s, p}\left(\partial \Omega, \mathbf{R}^{3}\right) \Rightarrow \boldsymbol{\varphi} \in \boldsymbol{B}^{s, p}\left(\partial \Omega, \mathbf{R}^{3}\right) .
$$

Furthermore, for any $t<s$, there exists a constant $C_{s, t}>0$ such that

$$
|\boldsymbol{\varphi}|_{s, p} \leq C_{s, t}\left(\left|\boldsymbol{T}_{\alpha} \boldsymbol{\varphi}\right|_{s, p}+|\boldsymbol{\varphi}|_{t, p}\right) .
$$

Proof. We prove that there exists a parametrix $\boldsymbol{S}_{\alpha}$ for the operator $\boldsymbol{T}_{\alpha}$ in the Hörmander class $\boldsymbol{L}_{1,1 / 2}^{0}\left(\partial \Omega, \mathbf{R}^{3}\right)$ of $3 \times 3$ matrix-valued, pseudo-differential operators on the boundary $\partial \Omega$. The proof of Lemma 5.2 is divided into three steps.

Step 1: The essential step in the proof is to verify the following matrix version of conditions (2.7a) and (2.7b) with $\mu:=0, \rho:=1$ and $\delta:=1 / 2$ (cf. Remark 2.2):

Lemma 5.3. Assume that condition (T) is satisfied. Then, for each point $x^{\prime}$ of $\partial \Omega$, we can find a neighborhood $U\left(x^{\prime}\right)$ of $x^{\prime}$ such that:

For any compact $K \subset U\left(x^{\prime}\right)$ and any multi-indices $\alpha, \beta$, there exist constants $C_{K, \alpha, \beta}>0$ and $C_{K}>0$ such that we have, for all $x^{\prime} \in K$ and all $\left|\xi^{\prime}\right| \geq C_{K}$,

$$
\begin{gathered}
\left\|D_{\xi^{\prime}}^{\alpha} D_{x^{\prime}}^{\beta} \boldsymbol{t}\left(x^{\prime}, \xi^{\prime}\right)\right\|\left\|\boldsymbol{t}\left(x^{\prime}, \xi^{\prime}\right)^{-1}\right\| \leq C_{K, \alpha, \beta}\left(1+\left|\xi^{\prime}\right|\right)^{-|\alpha|+(1 / 2)|\beta|}, \\
\left\|\boldsymbol{t}\left(x^{\prime}, \xi^{\prime}\right)^{-1}\right\| \leq C_{K} .
\end{gathered}
$$

Here $\|\cdot\|$ denotes a norm in the space of $3 \times 3$ matrices with complex entries.

Granting Lemma 5.3 for the moment, we shall prove Lemma 5.2.

Step 2: First, we cover the boundary $\partial \Omega$ by a finite number of local charts $\left\{\left(U_{j}, \chi_{j}\right)\right\}_{j=1}^{m}$ in each of which inequalities (5.3a) and (5.3b) hold true. Since the operator $\boldsymbol{T}_{\alpha}$ satisfies conditions $(2.7 \mathrm{a})$ and $(2.7 \mathrm{~b})$ of a matrix-valued version of Theorem 2.12 with $\mu:=0, \rho:=1$ and $\delta:=1 / 2$, it follows from an application of the same theorem that there exists a parametrix $\boldsymbol{S}_{\alpha}$ in the Hörmander class $\boldsymbol{L}_{1,1 / 2}^{0}\left(U_{j}, \mathbf{R}^{3}\right)$ for the operator $\boldsymbol{T}_{\alpha}$. Let $\left\{\phi_{j}\right\}_{j=1}^{m}$ be a partition of unity subordinate to the covering $\left\{U_{j}\right\}_{j=1}^{m}$, and choose a function $\psi_{j} \in C_{0}^{\infty}\left(U_{j}\right)$ such that $\psi_{j}=1$ on supp $\phi_{j}$, so that $\phi_{j} \psi_{j}=\phi_{j}$. 
Now we may assume that $\boldsymbol{\varphi} \in \boldsymbol{B}^{t, p}\left(\partial \Omega, \mathbf{R}^{3}\right)$ for some $t<s$ and that $\boldsymbol{T}_{\alpha} \boldsymbol{\varphi} \in$ $\boldsymbol{B}^{s, p}\left(\partial \Omega, \mathbf{R}^{3}\right)$. We remark that the operator $\boldsymbol{T}_{\alpha}$ can be written in the following form:

$$
\boldsymbol{T}_{\alpha}=\sum_{j=1}^{m} \phi_{j} \boldsymbol{T}_{\alpha} \psi_{j}+\sum_{j=1}^{m} \phi_{j} \boldsymbol{T}_{\alpha}\left(1-\psi_{j}\right) .
$$

However, the second terms $\phi_{j} \boldsymbol{T}_{\alpha}\left(1-\psi_{j}\right)$ are in $\boldsymbol{L}^{-\infty}\left(\partial \boldsymbol{\Omega}, \mathbf{R}^{3}\right)$, since $\phi_{j}\left(1-\psi_{j}\right)=0$. Hence we are reduced to the study of the first terms $\phi_{j} \boldsymbol{T}_{\alpha} \psi_{j}$. This implies that we have only to prove the following local version of assertions (5.1) and (5.2):

$$
\begin{aligned}
& \psi_{j} \boldsymbol{\varphi} \in \boldsymbol{B}^{t, p}\left(U_{j}, \mathbf{R}^{3}\right), \quad \boldsymbol{T}_{\alpha}\left(\psi_{j} \boldsymbol{\varphi}\right) \in \boldsymbol{B}^{s, p}\left(U_{j}, \mathbf{R}^{3}\right) \Rightarrow \psi_{j} \boldsymbol{\varphi} \in \boldsymbol{B}^{s, p}\left(U_{j}, \mathbf{R}^{3}\right) . \\
& \left|\psi_{j} \boldsymbol{\varphi}\right|_{s, p} \leq C_{s, t}\left(\left|\boldsymbol{T}_{\alpha}\left(\psi_{j} \boldsymbol{\varphi}\right)\right|_{s, p}^{2}+\left|\psi_{j} \boldsymbol{\varphi}\right|_{t, p}^{2}\right) .
\end{aligned}
$$

However, by applying Theorem 2.11 to our situation we obtain that the parametrix $\boldsymbol{S}_{\alpha}$ maps $\boldsymbol{B}_{\text {loc }}^{\sigma, p}\left(U_{j}, \mathbf{R}^{3}\right)$ continuously into itself for all $\sigma \in \mathbf{R}$. This proves the desired assertions $\left(5.1^{\prime}\right)$ and $\left(5.2^{\prime}\right)$, since we have the formula

$$
\psi_{j} \boldsymbol{\varphi} \equiv \boldsymbol{S}_{\alpha} \boldsymbol{T}_{\alpha}\left(\psi_{j} \boldsymbol{\varphi}\right) \bmod C^{\infty}\left(U_{j}, \mathbf{R}^{3}\right) .
$$

Lemma 5.2 (and hence Theorem 5.1) is proved, apart from the proof of Lemma 5.3.

\section{Step 3: Proof of Lemma 5.3}

By Theorem 4.8, we find that the symbol of $\boldsymbol{\Pi}$ has the following asymptotic expansion:

$$
\boldsymbol{p}_{1}\left(x^{\prime}, \xi^{\prime}\right)+\boldsymbol{p}_{0}\left(x^{\prime}, \xi^{\prime}\right)+\text { terms of order } \leq-1,
$$

where (cf. inequality (4.5))

$$
\boldsymbol{p}_{1}\left(x^{\prime}, \xi^{\prime}\right) \geq c_{0}\left|\xi^{\prime}\right| \boldsymbol{I} \quad \text { on } T^{*}(\partial \Omega) .
$$

Thus it follows that the symbol $\boldsymbol{t}\left(x^{\prime}, \xi^{\prime}\right)$ of the operator

$$
\boldsymbol{T}_{\alpha}=\alpha(x) \boldsymbol{\Pi}+(1-\alpha(x)) \boldsymbol{I}
$$

has the following asymptotic expansion:

$$
\begin{aligned}
\boldsymbol{t}\left(x^{\prime}, \xi^{\prime}\right)= & \alpha\left(x^{\prime}\right) \boldsymbol{p}_{1}\left(x^{\prime}, \xi^{\prime}\right)+\left[\left(1-\alpha\left(x^{\prime}\right)\right) \boldsymbol{I}+\alpha\left(x^{\prime}\right) \boldsymbol{p}_{0}\left(x^{\prime}, \xi\right)\right] \\
& + \text { terms of order } \leq-1
\end{aligned}
$$

Step 3-a: First, we verify condition (5.3b): 
By assertions (5.6) and (5.5), we can find a constant $0<c_{1}<1 / 2$ such that we have, for $\left|\xi^{\prime}\right|$ sufficiently large,

$$
\operatorname{Re} \boldsymbol{t}\left(x^{\prime}, \xi^{\prime}\right):=\frac{\boldsymbol{t}\left(x^{\prime}, \xi^{\prime}\right)+\boldsymbol{t}\left(x^{\prime}, \xi^{\prime}\right)^{*}}{2} \geq \begin{cases}\left(c_{0} \alpha\left(x^{\prime}\right)\left|\xi^{\prime}\right|+\frac{1}{4}\right) \boldsymbol{I} & \text { if } 0 \leq \alpha\left(x^{\prime}\right) \leq c_{1}, \\ \left(\frac{c_{0}}{2} \alpha\left(x^{\prime}\right)\left|\xi^{\prime}\right|+\frac{1}{8}\right) \boldsymbol{I} & \text { if } c_{1} \leq \alpha\left(x^{\prime}\right) \leq 1\end{cases}
$$

Hence there exists a constant $C_{1}>0$ such that we have, for $\left|\xi^{\prime}\right|$ sufficiently large,

$$
\operatorname{Re} t\left(x^{\prime}, \xi^{\prime}\right) \geq C_{1}\left(\alpha\left(x^{\prime}\right)\left|\xi^{\prime}\right|+1\right) \boldsymbol{I} .
$$

Inequality (5.7) implies condition (5.3b):

$$
\left\|\boldsymbol{t}\left(x^{\prime}, \xi^{\prime}\right)^{-1}\right\| \leq \frac{1}{C_{1}} .
$$

Indeed, it suffices to note the following:

$$
C_{1}\|\boldsymbol{u}\|^{2} \leq \operatorname{Re}\left(\boldsymbol{t}\left(x^{\prime}, \xi^{\prime}\right) \boldsymbol{u}, \boldsymbol{u}\right) \leq\left\|\boldsymbol{t}\left(x^{\prime}, \xi^{\prime}\right) \boldsymbol{u}\right\| \cdot\|\boldsymbol{u}\|, \quad \boldsymbol{u} \in \mathbf{C}^{3},
$$

so that

$$
C_{1}\|\boldsymbol{u}\| \leq\left\|\boldsymbol{t}\left(x^{\prime}, \xi^{\prime}\right) \boldsymbol{u}\right\|, \quad \boldsymbol{u} \in \mathbf{C}^{3} .
$$

Step 3-b: Secondly, we verify condition (5.3a) for $|\alpha|=1$ and $|\beta|=0$.

Since there exists a constant $C_{2}>0$ such that we have, for $\left|\xi^{\prime}\right|$ sufficiently large,

$$
\left\|D_{\xi^{\prime}}^{\alpha} t\left(x^{\prime}, \xi^{\prime}\right)\right\| \leq C_{2}\left(\alpha\left(x^{\prime}\right)+\left|\xi^{\prime}\right|^{-1}\right)
$$

it follows from inequality (5.7) that

$$
\begin{aligned}
\left\|D_{\xi^{\prime}}^{\alpha} \boldsymbol{t}\left(x^{\prime}, \xi^{\prime}\right)\right\| & \leq 2 C_{2}\left(1+\left|\xi^{\prime}\right|\right)^{-1}\left(\alpha\left(x^{\prime}\right)\left|\xi^{\prime}\right|+1\right) \\
& \leq \frac{2 C_{2}}{C_{1}}\left(1+\left|\xi^{\prime}\right|\right)^{-1}\left\|\boldsymbol{t}\left(x^{\prime}, \xi^{\prime}\right)\right\| .
\end{aligned}
$$

This inequality proves condition (5.3a) for $|\alpha|=1$ and $|\beta|=0$.

Step 3-c: We verify condition (5.3a) for $|\beta|=1$ and $|\alpha|=0$. To do this, we need the following elementary lemma on non-negative functions:

Lemma 5.4. Let $f(x)$ be a non-negative, $C^{2}$ function on $\mathbf{R}$ such that, for some constant $\gamma>0$,

$$
\sup _{x \in \mathbf{R}}\left|f^{\prime \prime}(x)\right| \leq \gamma
$$


Then we have the inequality

$$
\left|f^{\prime}(x)\right| \leq \sqrt{2 \gamma} \sqrt{f(x)} \text { on } \mathbf{R} .
$$

Since there exists a constant $C_{3}>0$ such that we have, for $\left|\xi^{\prime}\right|$ sufficiently large,

$$
\left\|D_{x^{\prime}}^{\beta} \boldsymbol{t}\left(x^{\prime}, \xi^{\prime}\right)\right\| \leq C_{3}\left(\left|D_{x^{\prime}}^{\beta} \alpha\left(x^{\prime}\right)\right| \cdot\left|\xi^{\prime}\right|+\alpha\left(x^{\prime}\right)\left|\xi^{\prime}\right|+1\right),
$$

it follows from an application of Lemma 5.4 and inequalities (5.7) and (5.8) that, for some constant $C_{4}>0$,

$$
\begin{aligned}
\left\|D_{x^{\prime}}^{\beta} \boldsymbol{t}\left(x^{\prime}, \xi^{\prime}\right)\right\| & \leq C_{4}\left\{\left(\sqrt{\alpha\left(x^{\prime}\right)}\left|\xi^{\prime}\right|+1\right)+\left(\alpha\left(x^{\prime}\right)\left|\xi^{\prime}\right|+1\right)\right\} \\
& \leq C_{4}\left\{\left|\xi^{\prime}\right|^{1 / 2}\left(\alpha\left(x^{\prime}\right)\left|\xi^{\prime}\right|+1\right)^{1 / 2}+\left(\alpha\left(x^{\prime}\right)\left|\xi^{\prime}\right|+1\right)\right\} \\
& \leq \frac{C_{4}}{C_{1}}\left\|\boldsymbol{t}\left(x^{\prime}, \xi^{\prime}\right)\right\|\left(\left|\xi^{\prime}\right|^{1 / 2} \sqrt{C_{1}}\left\|\boldsymbol{t}\left(x^{\prime}, \xi^{\prime}\right)\right\|^{-1 / 2}+1\right) \\
& \leq \frac{2 C_{4}}{C_{1}}\left\|\boldsymbol{t}\left(x^{\prime}, \xi^{\prime}\right)\right\|\left(1+\left|\xi^{\prime}\right|\right)^{1 / 2}
\end{aligned}
$$

This inequality proves condition (5.3a) for $|\beta|=1$ and $|\alpha|=0$.

Step 3-d: Similarly, we can verify condition (5.3a) for the general case: $|\alpha|+|\beta|=k, k \in \mathbf{N}$.

The proof of Lemma 5.3 is complete.

Now the proof of Lemma 5.2 and hence that of Theorem 5.1 is complete.

\section{Uniqueness Theorem for Problem (1.4)}

We associate with problem (1.4) a linear operator

$$
\mathscr{A}_{\alpha}=\left(\boldsymbol{A}, \boldsymbol{B}_{\alpha}\right): \boldsymbol{H}^{s, p}\left(\Omega, \mathbf{R}^{3}\right) \mapsto \boldsymbol{H}^{s-2, p}\left(\Omega, \mathbf{R}^{3}\right) \times \boldsymbol{B}_{(\alpha)}^{s-1-1 / p, p}\left(\partial \Omega, \mathbf{R}^{3}\right) .
$$

Then the next uniqueness theorem for problem (1.4) asserts that the operator $\mathscr{A}_{\alpha}$ is injective, that is, we have the assertion

$$
\operatorname{dim} N\left(\mathscr{A}_{\alpha}\right)=0
$$

THEOREM 6.1. Let $1<p<\infty$ and $s>1 / p+1$. Assume that conditions (T) and (A) are satisfied. If a function $\boldsymbol{v} \in \boldsymbol{H}^{s, p}\left(\Omega, \mathbf{R}^{3}\right)$ is a solution of the mixed displacement-traction problem 


$$
\begin{cases}\operatorname{div}(\mathbf{a}(x) \cdot \nabla \boldsymbol{v})=\mathbf{0} & \text { in } \boldsymbol{\Omega}, \\ \alpha(x)(\mathbf{a}(x) \cdot \nabla \boldsymbol{v} \cdot \boldsymbol{n})+(1-\alpha(x)) \boldsymbol{v}=\mathbf{0} & \text { on } \partial \Omega\end{cases}
$$

then it follows that $\boldsymbol{v}=\mathbf{0}$ in $\Omega$.

Proof. First, by Theorem 5.1 we may assume that

$$
v \in C^{\infty}\left(\bar{\Omega}, \mathbf{R}^{3}\right)
$$

Moreover, it should be noticed that the homogeneous boundary condition

$$
\alpha(x)(\mathbf{a}(x) \cdot \nabla \boldsymbol{v} \cdot \boldsymbol{n})+(1-\alpha(x)) \boldsymbol{v}=\mathbf{0} \quad \text { on } \partial \boldsymbol{\Omega}
$$

includes the condition

$$
\boldsymbol{v}=\mathbf{0} \quad \text { on the set } \Gamma_{0}:=\{x \in \partial \Omega: \alpha(x)=0\} .
$$

Hence it follows from an application of Green's formula (4.3) that

$$
\begin{aligned}
0 & =\int_{\Omega} \boldsymbol{\nabla} \boldsymbol{v} \cdot \mathbf{a}(x) \cdot \nabla \boldsymbol{v} d x-\int_{\partial \Omega} \boldsymbol{v}[\mathbf{a}(x) \cdot \nabla \boldsymbol{v} \cdot \boldsymbol{n}] d a \\
& =\int_{\Omega} \boldsymbol{e}(\boldsymbol{v}) \cdot \mathbf{a}(x) \cdot \boldsymbol{e}(\boldsymbol{v}) d x-\int_{\partial \Omega \backslash \Gamma_{0}} \boldsymbol{v}[\mathbf{a}(x) \cdot \nabla \boldsymbol{v} \cdot \boldsymbol{n}] d a \\
& =\int_{\Omega} \boldsymbol{e}(\boldsymbol{v}) \cdot \mathbf{a}(x) \cdot \boldsymbol{e}(\boldsymbol{v}) d x+\int_{\partial \Omega \backslash \Gamma_{0}}\left(\frac{1-\alpha(x)}{\alpha(x)}\right)\|\boldsymbol{v}\|^{2} d a \\
& \geq \int_{\Omega} \boldsymbol{e}(\boldsymbol{v}) \cdot \mathbf{a}(x) \cdot \boldsymbol{e}(\boldsymbol{v}) d x
\end{aligned}
$$

where $\boldsymbol{e}(\boldsymbol{v})=\left(e_{i j}(v)\right)$ is the linearized strain tensor associated with the function $\boldsymbol{v}$. However, since the elasticity tensor $\mathbf{a}(x)$ is uniformly pointwise stable, it follows that

$$
\frac{1}{2} \boldsymbol{e}(\boldsymbol{v}) \cdot \mathbf{a}(x) \cdot \boldsymbol{e}(\boldsymbol{v}) \geq \eta\|\boldsymbol{e}(\boldsymbol{v})\|^{2}, \quad x \in \bar{\Omega} .
$$

Hence we have the inequality

$$
0 \geq 2 \eta \int_{\Omega}\|\boldsymbol{e}(\boldsymbol{v})\|^{2} d x, \quad x \in \bar{\Omega}
$$

and so

$$
\boldsymbol{e}(\boldsymbol{v})=\mathbf{0} \quad \text { in } \Omega .
$$


This implies that

$$
\begin{aligned}
\mathbf{0} & =\alpha(x)(\mathbf{a}(x) \cdot \nabla \boldsymbol{v} \cdot \boldsymbol{n})+(1-\alpha(x)) \boldsymbol{v} \\
& =\alpha(x)(\mathbf{a}(x) \cdot \boldsymbol{e}(\boldsymbol{v}) \cdot \boldsymbol{n})+(1-\alpha(x)) \boldsymbol{v} \\
& =(1-\alpha(x)) \boldsymbol{v} \quad \text { on } \partial \Omega .
\end{aligned}
$$

Thus, if we let

$$
\gamma=\{x \in \partial \Omega: \alpha(x)<1\}
$$

we find that

$$
\boldsymbol{v}=\mathbf{0} \quad \text { on } \gamma
$$

Furthermore, condition (A) implies that the open set $\gamma$ is non-empty.

Therefore, we can make use of the first Korn inequality (4.4) to obtain that

$$
\boldsymbol{v}=\mathbf{0} \quad \text { in } \Omega .
$$

Indeed, we have, by assertion (6.1) and inequality (4.4) with $\boldsymbol{u}:=\boldsymbol{v}$,

$$
0=2 \eta \int_{\Omega}\|\boldsymbol{e}(\boldsymbol{v})\|^{2} d x \geq 2 \eta c(\gamma)\left(\int_{\Omega}\|\boldsymbol{v}\|^{2} d x+\int_{\Omega}\|\boldsymbol{\nabla} \boldsymbol{v}\|^{2} d x\right)
$$

which proves the desired assertion (6.2).

The proof of Theorem 6.1 is complete.

\section{Existence Theorem for Problem (1.4)}

The next existence theorem for problem (1.4) asserts that the operator $\mathscr{A}_{\alpha}$ is surjective, that is, we have the assertion

$$
\operatorname{codim} R\left(\mathscr{A}_{\alpha}\right)=0 \text {. }
$$

THEOREM 7.1. Let $1<p<\infty$ and $s>1 / p+1$. If conditions (T) and (A) are satisfied, then, for any $\boldsymbol{f} \in \boldsymbol{H}^{s-2, p}\left(\boldsymbol{\Omega}, \mathbf{R}^{3}\right)$ and any $\boldsymbol{\varphi} \in \boldsymbol{B}_{(\alpha)}^{s-1-1 / p, p}\left(\partial \Omega, \mathbf{R}^{3}\right)$, the mixed displacement-traction problem

$$
\begin{cases}\operatorname{div}(\mathbf{a}(x) \cdot \nabla \boldsymbol{v})=\boldsymbol{f} & \text { in } \boldsymbol{\Omega} \\ \alpha(x)(\mathbf{a}(x) \cdot \nabla \boldsymbol{v} \cdot \boldsymbol{n})+(1-\alpha(x)) \boldsymbol{v}=\boldsymbol{\varphi} & \text { on } \partial \boldsymbol{\Omega}\end{cases}
$$

has a solution $\boldsymbol{v} \in \boldsymbol{H}^{s, p}\left(\Omega, \mathbf{R}^{3}\right)$. 


\subsection{Proof of Theorem 7.1}

By Theorem 4.4, we know that

$$
\text { ind } \mathscr{A}_{\alpha}=\text { ind } \mathscr{T}_{\alpha} \text {, }
$$

where the operator

$$
\mathscr{T}_{\alpha}: \boldsymbol{B}^{s-1 / p, p}\left(\partial \Omega, \mathbf{R}^{3}\right) \rightarrow \boldsymbol{B}^{s-1 / p, p}\left(\partial \Omega, \mathbf{R}^{3}\right)
$$

is defined as follows:

(a) The domain $D\left(\mathscr{T}_{\alpha}\right)$ of $\mathscr{T}_{\alpha}$ is the space

$$
D\left(\mathscr{T}_{\alpha}\right)=\left\{\boldsymbol{\varphi} \in \boldsymbol{B}^{s-1 / p, p}\left(\partial \boldsymbol{\Omega}, \mathbf{R}^{3}\right): \boldsymbol{T}_{\alpha} \boldsymbol{\varphi} \in B^{s-1 / p, p}\left(\partial \boldsymbol{\Omega}, \mathbf{R}^{3}\right)\right\} .
$$

(b) $\mathscr{T}_{\alpha} \varphi=\boldsymbol{T}_{\alpha} \boldsymbol{\varphi}, \boldsymbol{\varphi} \in D\left(\mathscr{T}_{\alpha}\right)$.

However, Theorem 6.1 asserts that the operator $\mathscr{A}_{\alpha}$ is injective. By using again Theorem 4.4, we obtain that

$$
\operatorname{dim} N\left(\mathscr{A}_{\alpha}\right)=\operatorname{dim} N\left(\mathscr{T}_{\alpha}\right)=0 .
$$

Hence, in order to prove the surjectivity of $\mathscr{A}_{\alpha}$, or equivalently,

$$
\operatorname{codim} R\left(\mathscr{A}_{\alpha}\right)=\operatorname{codim} R\left(\mathscr{T}_{\alpha}\right)=0,
$$

it suffices to show the following:

Proposition 7.2. The index of the operator $\mathscr{T}_{\alpha}$ is equal to zero, that is,

$$
\text { ind } \mathscr{T}_{\alpha}=\operatorname{dim} N\left(\mathscr{T}_{\alpha}\right)-\operatorname{codim} R\left(\mathscr{T}_{\alpha}\right)=0 \text {. }
$$

Proof. The proof of Proposition 7.2 is divided into three steps.

Step 1: First, we replace the operator $\boldsymbol{A}$ by the operator $\boldsymbol{A}-\lambda \boldsymbol{I}$ with $\lambda \geq 0$, and consider instead of problem (1.4) the following boundary value problem:

$$
\begin{cases}(\boldsymbol{A}-\lambda \boldsymbol{I}) \boldsymbol{u}=\boldsymbol{f} & \text { in } \Omega, \\ \boldsymbol{B}_{\alpha} \boldsymbol{u}=\alpha(x) \boldsymbol{B} \boldsymbol{u}+(1-\alpha(x)) \boldsymbol{u}=\boldsymbol{\varphi} & \text { on } \partial \Omega .\end{cases}
$$

We associate with problem $(1.5)_{\lambda}$ a linear operator

$$
\mathscr{A}_{\alpha}(\lambda)=\left(\boldsymbol{A}-\lambda \boldsymbol{I}, \boldsymbol{B}_{\alpha}\right): \boldsymbol{H}^{s, p}\left(\boldsymbol{\Omega}, \mathbf{R}^{3}\right) \mapsto \boldsymbol{H}^{s-2, p}\left(\Omega, \mathbf{R}^{3}\right) \times \boldsymbol{B}_{(\alpha)}^{s-1-1 / p, p}\left(\partial \Omega, \mathbf{R}^{3}\right) .
$$

It should be noticed that the operator $\mathscr{A}_{\alpha}(\lambda)$ coincides with the operator $\mathscr{A}_{\alpha}$ when $\lambda=0$, that is, $\mathscr{A}_{\alpha}(0)=\mathscr{A}_{\alpha}$.

We reduce the study of problem $(1.5)_{\lambda}$ to that of a $3 \times 3$ matrix-valued, pseudo-differential operator on the boundary, just as in the proof of Theorem 5.1. 
We can prove that Theorem 4.1 remains valid for the operator $\boldsymbol{A}-\lambda \boldsymbol{I}$. More precisely, we have the following two assertions (a) and (b):

(a) The pure displacement problem (Dirichlet problem)

$$
\begin{cases}(\boldsymbol{A}-\lambda \boldsymbol{I}) \boldsymbol{w}=\mathbf{0} & \text { in } \Omega, \\ \boldsymbol{w}=\boldsymbol{\varphi} & \text { on } \partial \Omega\end{cases}
$$

has a unique solution $\boldsymbol{w}$ in $\boldsymbol{H}^{t, p}\left(\Omega, \mathbf{R}^{3}\right)$ for any $\boldsymbol{\varphi} \in \boldsymbol{B}^{t-1 / p, p}\left(\partial \Omega, \mathbf{R}^{3}\right)(t \in \mathbf{R})$.

(b) The Poisson operator

$$
\mathscr{P}(\lambda): \boldsymbol{B}^{t-1 / p, p}\left(\partial \Omega, \mathbf{R}^{3}\right) \rightarrow \boldsymbol{H}^{t, p}\left(\Omega, \mathbf{R}^{3}\right),
$$

defined by the formula $\boldsymbol{w}=\mathscr{P}(\lambda) \boldsymbol{\varphi}$, is an isomorphism of $\boldsymbol{B}^{t-1 / p, p}\left(\partial \Omega, \mathbf{R}^{3}\right)$ onto the null space $N(\boldsymbol{A}-\lambda \boldsymbol{I}, t, p)=\left\{\boldsymbol{u} \in \boldsymbol{H}^{t, p}\left(\Omega, \mathbf{R}^{3}\right):(\boldsymbol{A}-\lambda \boldsymbol{I}) \boldsymbol{u}=\mathbf{0}\right.$ in $\left.\Omega\right\}$ for all $t \in \mathbf{R}$; and its inverse is the trace operator on $\partial \Omega$.

Let $\boldsymbol{T}_{\alpha}(\lambda)$ be a $3 \times 3$ matrix-valued, classical pseudo-differential operator of first order on the boundary $\partial \Omega$ defined as follows:

$$
\boldsymbol{T}_{\alpha}(\lambda):=\boldsymbol{B}_{\alpha} \mathscr{P}(\lambda)=\alpha(x) \boldsymbol{\Pi}(\lambda)+(1-\alpha(x)) \boldsymbol{I}, \quad \lambda \geq 0,
$$

where

$$
\begin{gathered}
\boldsymbol{\Pi}(\lambda): \boldsymbol{C}^{\infty}\left(\partial \Omega, \mathbf{R}^{3}\right) \rightarrow \boldsymbol{C}^{\infty}\left(\partial \Omega, \mathbf{R}^{3}\right) \\
\boldsymbol{\varphi} \mapsto \boldsymbol{B}_{\alpha}(\mathscr{P}(\lambda) \boldsymbol{\varphi})
\end{gathered}
$$

Since the operator $\boldsymbol{T}_{\alpha}(\lambda): \boldsymbol{C}^{\infty}\left(\partial \Omega, \mathbf{R}^{3}\right) \rightarrow \boldsymbol{C}^{\infty}\left(\partial \boldsymbol{\Omega}, \mathbf{R}^{3}\right)$ extends to a continuous linear operator $\boldsymbol{T}_{\alpha}(\lambda): \boldsymbol{B}^{t, p}\left(\partial \boldsymbol{\Omega}, \mathbf{R}^{3}\right) \rightarrow \boldsymbol{B}^{t-1, p}\left(\partial \boldsymbol{\Omega}, \mathbf{R}^{3}\right)$ for all $t \in \mathbf{R}$, we can introduce a densely defined, closed linear operator

$$
\mathscr{T}_{\alpha}(\lambda): \boldsymbol{B}^{s-1 / p, p}\left(\partial \Omega, \mathbf{R}^{3}\right) \rightarrow \boldsymbol{B}^{s-1 / p, p}\left(\partial \Omega, \mathbf{R}^{3}\right)
$$

as follows.

$(\alpha)$ The domain $D\left(\mathscr{T}_{\alpha}(\lambda)\right)$ of $\mathscr{T}_{\alpha}(\lambda)$ is the space

$$
D\left(\mathscr{T}_{\alpha}(\lambda)\right)=\left\{\boldsymbol{\varphi} \in \boldsymbol{B}^{s-1 / p, p}\left(\partial \Omega, \mathbf{R}^{3}\right): \boldsymbol{T}_{\alpha}(\lambda) \boldsymbol{\varphi} \in \boldsymbol{B}^{s-1 / p, p}\left(\partial \Omega, \mathbf{R}^{3}\right)\right\} .
$$

$(\beta) \mathscr{T}_{\alpha}(\lambda) \boldsymbol{\varphi}=\boldsymbol{T}_{\alpha}(\lambda) \boldsymbol{\varphi}, \boldsymbol{\varphi} \in D\left(\mathscr{T}_{\alpha}(\lambda)\right)$.

It should be noticed that the operator $\mathscr{T}_{\alpha}(\lambda)$ coincides with the operator $\mathscr{T}_{\alpha}$ when $\lambda=0$, that is, $\mathscr{T}_{\alpha}(0)=\mathscr{T}_{\alpha}$.

Then we can obtain the following three assertions (I), (II) and (III), analogous to Theorem 4.4:

(I) The null space $N\left(\mathscr{A}_{\alpha}(\lambda)\right)$ of $\mathscr{A}_{\alpha}(\lambda)$ has finite dimension if and only if the null space $N\left(\mathscr{T}_{\alpha}(\lambda)\right)$ of $\mathscr{T}_{\alpha}(\lambda)$ has finite dimension, and we have the formula 


$$
\operatorname{dim} N\left(\mathscr{A}_{\alpha}(\lambda)\right)=\operatorname{dim} N\left(\mathscr{T}_{\alpha}(\lambda)\right) .
$$

(II) The range $R\left(\mathscr{A}_{\alpha}(\lambda)\right)$ of $\mathscr{A}_{\alpha}(\lambda)$ is closed if and only if the range $R\left(\mathscr{T}_{\alpha}(\lambda)\right)$ of $\mathscr{T}_{\alpha}(\lambda)$ is closed; and $R\left(\mathscr{A}_{\alpha}(\lambda)\right)$ has finite codimension if and only if $R\left(\mathscr{T}_{\alpha}(\lambda)\right)$ has finite codimension, and we have the formula

$$
\operatorname{codim} R\left(\mathscr{A}_{\alpha}(\lambda)\right)=\operatorname{codim} R\left(\mathscr{T}_{\alpha}(\lambda)\right) .
$$

(III) The operator $\mathscr{A}_{\alpha}(\lambda)$ is a Fredholm operator if and only if the operator $\mathscr{T}_{\alpha}(\lambda)$ is a Fredholm operator, and we have the formula

$$
\text { ind } \mathscr{A}_{\alpha}(\lambda)=\text { ind } \mathscr{T}_{\alpha}(\lambda) \text {. }
$$

Step 2: To study problem $(1.5)_{\lambda}$, we shall make use of a method essentially due to Agmon (cf. [Ag], [LM] and also [Ta1, Section 8.4]). This is a technique of treating a spectral parameter $\lambda \boldsymbol{I}$ as a second-order differential operator of an extra variable and relating the old problem to a new one with the additional variable.

We introduce an auxiliary variable $y$ of the unit circle

$$
S=\mathbf{R} / 2 \pi \mathbf{Z},
$$

and replace the parameter $-\lambda \boldsymbol{I}$ by the second-order differential operator

$$
\frac{\partial^{2}}{\partial y^{2}} \boldsymbol{I}
$$

Namely, we replace the operator $\boldsymbol{A}-\lambda \boldsymbol{I}$ by the operator

$$
\tilde{\boldsymbol{\Lambda}}=\boldsymbol{A}+\frac{\partial^{2}}{\partial y^{2}} \boldsymbol{I},
$$

and consider instead of problem $(1.5)_{\lambda}$ the following boundary value problem:

$$
\begin{cases}\tilde{\boldsymbol{\Lambda}} \tilde{\boldsymbol{u}}=\left(\boldsymbol{A}+\frac{\partial^{2}}{\partial y^{2}} \boldsymbol{I}\right) \tilde{\boldsymbol{u}}=\tilde{\boldsymbol{f}} & \text { in } \boldsymbol{\Omega} \times S, \\ \boldsymbol{B}_{\alpha} \tilde{\boldsymbol{u}}=\alpha(x) \boldsymbol{B} \tilde{\boldsymbol{u}}+(1-\alpha(x)) \tilde{\boldsymbol{u}}=\tilde{\boldsymbol{\varphi}} & \text { on } \partial \boldsymbol{\Omega} \times S .\end{cases}
$$

We can prove that Theorem 4.1 remains valid for the operator $\tilde{\boldsymbol{\Lambda}}=$ $\boldsymbol{A}+\partial^{2} / \partial y^{2} \boldsymbol{I}$. More precisely, we have the following two assertions $(\tilde{\boldsymbol{a}})$ and $(\tilde{b})$ :

(a) The pure displacement problem (Dirichlet problem)

$$
\begin{cases}\tilde{\boldsymbol{\Lambda}} \tilde{\boldsymbol{w}}=\mathbf{0} & \text { in } \Omega \times S, \\ \tilde{\boldsymbol{w}}=\tilde{\boldsymbol{\varphi}} & \text { on } \partial \Omega \times S\end{cases}
$$


has a unique solution $\tilde{\boldsymbol{w}}$ in $\boldsymbol{H}^{t, p}\left(\boldsymbol{\Omega} \times S, \mathbf{R}^{3}\right)$ for any $\tilde{\boldsymbol{\varphi}} \in \boldsymbol{B}^{t-1 / p, p}\left(\partial \boldsymbol{\Omega} \times S, \mathbf{R}^{3}\right)$ $(t \in \mathbf{R})$.

( $\tilde{b})$ The Poisson operator

$$
\tilde{\mathscr{P}}: \boldsymbol{B}^{t-1 / p, p}\left(\partial \Omega \times S, \mathbf{R}^{3}\right) \rightarrow \boldsymbol{H}^{t, p}\left(\Omega \times S, \mathbf{R}^{3}\right),
$$

defined by the formula $\tilde{\boldsymbol{w}}:=\tilde{\mathscr{P}} \tilde{\boldsymbol{\varphi}}$, is an isomorphism of $\boldsymbol{B}^{t-1 / p, p}\left(\partial \boldsymbol{\Omega} \times S, \mathbf{R}^{3}\right)$ onto the null space $N(\tilde{\boldsymbol{\Lambda}}, t, p)=\left\{\tilde{\boldsymbol{u}} \in \boldsymbol{H}^{t, p}\left(\Omega \times S, \mathbf{R}^{3}\right): \tilde{\boldsymbol{\Lambda}} \tilde{\boldsymbol{u}}=\mathbf{0}\right.$ in $\left.\Omega \times S\right\}$ for all $t \in \mathbf{R}$; and its inverse is the trace operator on $\partial \Omega \times S$.

We let

$$
\begin{gathered}
\tilde{\boldsymbol{T}}_{\alpha}: \boldsymbol{C}^{\infty}\left(\partial \boldsymbol{\Omega} \times S, \mathbf{R}^{3}\right) \rightarrow \boldsymbol{C}^{\infty}\left(\partial \Omega \times S, \mathbf{R}^{3}\right) \\
\tilde{\boldsymbol{\varphi}} \mapsto \boldsymbol{B}_{\alpha}(\tilde{\mathscr{P}} \tilde{\boldsymbol{\varphi}})
\end{gathered}
$$

Then the operator $\tilde{\boldsymbol{T}}_{\alpha}$ can be decomposed as follows:

$$
\tilde{\boldsymbol{T}}_{\alpha}=\alpha(x) \tilde{\boldsymbol{\Pi}}+(1-\alpha(x)) \boldsymbol{I},
$$

where

$$
\tilde{\boldsymbol{\Pi}} \tilde{\boldsymbol{\varphi}}=\boldsymbol{B}(\tilde{\mathscr{P}} \tilde{\boldsymbol{\varphi}})=\left.\mathbf{a}(x) \cdot \nabla(\tilde{\mathscr{P}} \tilde{\boldsymbol{\varphi}}) \cdot \boldsymbol{n}\right|_{\partial \boldsymbol{\Omega} \times S} .
$$

The operator $\tilde{\boldsymbol{\Pi}}$ is a $3 \times 3$ matrix-valued, classical pseudo-differential operator of first order on the boundary $\partial \Omega \times S$, and its symbol is given by the following formula:

$$
\begin{aligned}
\tilde{\boldsymbol{t}}\left(x^{\prime}, \xi^{\prime}, y, \eta\right)= & \alpha\left(x^{\prime}\right) \tilde{\boldsymbol{p}}_{1}\left(x^{\prime}, \xi^{\prime}, y, \eta\right)+\left[\left(1-\alpha\left(x^{\prime}\right)\right) \boldsymbol{I}+\alpha\left(x^{\prime}\right) \tilde{\boldsymbol{p}}_{0}\left(x^{\prime}, \xi^{\prime}, y, \eta\right)\right] \\
& + \text { terms of order } \leq-1
\end{aligned}
$$

where (cf. inequality (5.5))

$$
\tilde{\boldsymbol{p}}_{1}\left(x^{\prime}, \xi^{\prime}, y, \eta\right) \geq \tilde{c}_{0} \sqrt{\left|\xi^{\prime}\right|^{2}+\eta^{2} \boldsymbol{I}} \text { on } T^{*}(\partial \Omega \times S) .
$$

Thus we find that the operator

$$
\tilde{\boldsymbol{T}}_{\alpha}=\alpha\left(x^{\prime}\right) \tilde{\boldsymbol{\Pi}}+\left(1-\alpha\left(x^{\prime}\right)\right) \boldsymbol{I}
$$

is a $3 \times 3$ matrix-valued, classical pseudo-differential operator of first order on the boundary $\partial \Omega \times S$ and its symbol is given by the following formula:

$$
\begin{aligned}
\boldsymbol{t}\left(x^{\prime}, \xi^{\prime}\right)= & \alpha\left(x^{\prime}\right) \boldsymbol{p}_{1}\left(x^{\prime}, \xi^{\prime}\right)+\left[\left(1-\alpha\left(x^{\prime}\right)\right) \boldsymbol{I}+\alpha\left(x^{\prime}\right) \boldsymbol{p}_{0}\left(x^{\prime}, \xi\right)\right] \\
& + \text { terms of order } \leq-1
\end{aligned}
$$


Then, by virtue of assertions (7.2) and (7.1) it is easy to verify that the operator $\tilde{\boldsymbol{T}}_{\alpha}$ satisfies conditions (2.7a) and (2.7b) of a matrix-valued version of Theorem 2.12 with $\mu:=0, \rho:=1$ and $\delta:=1 / 2$, just as in the proof of Lemma 5.3. Hence there exists a parametrix $\tilde{\boldsymbol{S}}_{\alpha}$ in the Hörmander class $\boldsymbol{L}_{1,1 / 2}^{0}\left(\partial \boldsymbol{\Omega} \times S, \mathbf{R}^{3}\right)$ for the operator $\tilde{\boldsymbol{T}}_{\alpha}$.

Therefore, we obtain the following result, analogous to Lemma 5.2:

Lemma 7.3. If condition ( $\mathrm{T}$ ) is satisfied, then we have, for all $s \in \mathbf{R}$,

$$
\tilde{\boldsymbol{\varphi}} \in \mathscr{D}^{\prime}(\partial \boldsymbol{\Omega} \times S), \quad \tilde{\boldsymbol{T}}_{\alpha} \tilde{\boldsymbol{\varphi}} \in \boldsymbol{B}^{s, p}\left(\partial \boldsymbol{\Omega} \times S, \mathbf{R}^{3}\right) \Rightarrow \tilde{\boldsymbol{\varphi}} \in \boldsymbol{B}^{s, p}\left(\partial \Omega \times S, \mathbf{R}^{3}\right) .
$$

Furthermore, for any $t<s$, there exists a constant $\tilde{C}_{s, t}>0$ such that

$$
|\tilde{\boldsymbol{\varphi}}|_{s, p} \leq \tilde{C}_{s, t}\left(\left|\tilde{\boldsymbol{T}}_{\alpha} \tilde{\boldsymbol{\varphi}}\right|_{s, p}+|\tilde{\boldsymbol{\varphi}}|_{t, p}\right) .
$$

We introduce a densely defined, closed linear operator

$$
\tilde{\mathscr{T}}_{\alpha}: \boldsymbol{B}^{s-1 / p, p}\left(\partial \Omega \times S, \mathbf{R}^{3}\right) \rightarrow \boldsymbol{B}^{s-1 / p, p}\left(\partial \Omega \times S, \mathbf{R}^{3}\right)
$$

as follows.

( $\tilde{\alpha})$ The domain $D\left(\tilde{\mathscr{T}}_{\alpha}\right)$ of $\tilde{\mathscr{T}}_{\alpha}$ is the space

$$
D\left(\tilde{\mathscr{T}}_{\alpha}\right)=\left\{\tilde{\boldsymbol{\varphi}} \in \boldsymbol{B}^{s-1 / p, p}\left(\partial \boldsymbol{\Omega} \times S, \mathbf{R}^{3}\right): \tilde{\boldsymbol{T}}_{\alpha} \tilde{\boldsymbol{\varphi}} \in \boldsymbol{B}^{s-1 / p, p}\left(\partial \boldsymbol{\Omega} \times S, \mathbf{R}^{3}\right)\right\} .
$$

$(\tilde{\boldsymbol{\beta}}) \tilde{\mathscr{T}}_{\alpha} \tilde{\boldsymbol{\varphi}}=\tilde{\boldsymbol{T}}_{\alpha} \tilde{\boldsymbol{\varphi}}, \tilde{\boldsymbol{\varphi}} \in D\left(\tilde{\mathscr{T}}_{\alpha}\right)$.

Then the most fundamental relationship between the operators $\tilde{\mathscr{T}}_{\alpha}$ and $\mathscr{T}_{\alpha}(\lambda)$ $(\lambda \geq 0)$ is the following:

Proposition 7.4. If ind $\tilde{\mathscr{T}}_{\alpha}$ is finite, then there exists a finite subset $K$ of $\mathbf{Z}$ such that the operator $\mathscr{T}_{\alpha}\left(\lambda^{\prime}\right)$ is bijective for all $\lambda^{\prime}=\ell^{2}$ satisfying $\ell \in \mathbf{Z} \backslash K$.

Granting Proposition 7.4 for the moment, we shall prove Proposition 7.2 (and hence Theorem 7.1).

\section{Step 3: End of Proof of Proposition 7.2}

Step 3-a: We show that if condition $(T)$ is satisfied, then we have the assertion

$$
\text { ind } \tilde{\mathscr{T}}_{\alpha}=\operatorname{dim} N\left(\tilde{\mathscr{T}}_{\alpha}\right)-\operatorname{codim} R\left(\tilde{\mathscr{T}}_{\alpha}\right)<\infty
$$

To this end, we need a useful criterion for Fredholm operators (cf. [Ta1, Theorem 3.7.6]): 
Lemma 7.5 (Peetre). Let $X, Y, Z$ be Banach spaces such that $X \subset Z$ is a compact injection, and let $T$ be a closed linear operator from $X$ into $Y$ with domain $D(T)$. Then the following two conditions (i) and (ii) are equivalent:

(i) The null space $N(T)$ of $T$ has finite dimension and the range $R(T)$ of $T$ is closed in $Y$.

(ii) There is a constant $C>0$ such that

$$
\|x\|_{X} \leq C\left(\|T x\|_{Y}+\|x\|_{Z}\right), \quad x \in D(T) .
$$

Now, estimate (7.3) gives that we have, for $t<s-1 / p$,

$$
|\tilde{\boldsymbol{\varphi}}|_{s-1 / p, p} \leq \tilde{C}_{t}\left(|\tilde{\boldsymbol{T}} \tilde{\boldsymbol{\varphi}}|_{s-1 / p, p}+|\tilde{\boldsymbol{\varphi}}|_{t, p}\right), \quad \tilde{\boldsymbol{\varphi}} \in D\left(\tilde{\mathscr{T}}_{\alpha}\right) .
$$

However, it follows from an application of the Rellich-Kondrachov theorem that the injection $\boldsymbol{B}^{s-1 / p, p}\left(\partial \Omega \times S, \mathbf{R}^{3}\right) \rightarrow \boldsymbol{B}^{t, p}\left(\partial \Omega \times S, \mathbf{R}^{3}\right)$ is compact (or completely continuous) for $t<s-1 / p$. Thus, by applying Lemma 7.5 with

$$
\begin{aligned}
X & =Y:=\boldsymbol{B}^{s-1 / p, p}\left(\partial \Omega \times S, \mathbf{R}^{3}\right), \\
Z & :=\boldsymbol{B}^{t, p}\left(\partial \Omega \times S, \mathbf{R}^{3}\right), \\
T & :=\tilde{\mathscr{T}}_{\alpha},
\end{aligned}
$$

we obtain that the range $R\left(\tilde{\mathscr{T}}_{\alpha}\right)$ is closed in $\boldsymbol{B}^{s-1 / p, p}\left(\partial \Omega \times S, \mathbf{R}^{3}\right)$ and that

$$
\operatorname{dim} N\left(\tilde{\mathscr{T}}_{\alpha}\right)<\infty .
$$

On the other hand, by formula (7.2) we find that the symbol of the adjoint $\tilde{\boldsymbol{T}}^{*}$ is given by the following formula (cf. Theorem 2.7):

$$
\begin{aligned}
& \alpha\left(x^{\prime}\right)\left(\tilde{\boldsymbol{p}}_{1}\left(x^{\prime}, \xi^{\prime}, y, \eta\right)-\sqrt{-1} \tilde{\boldsymbol{q}}_{1}\left(x^{\prime}, \xi^{\prime}, y, \eta\right)\right) \\
& \quad+\left(\left[1-\alpha\left(x^{\prime}\right)+\alpha\left(x^{\prime}\right) \tilde{\boldsymbol{p}}_{0}\left(x^{\prime}, \xi^{\prime}, y, \eta\right)-\sum_{j=1}^{2} \partial_{x_{j}}\left(\alpha\left(x^{\prime}\right) \cdot \partial_{\xi_{j}} \tilde{\boldsymbol{q}}_{1}\left(x^{\prime}, \xi^{\prime}, y, \eta\right)\right)\right]\right. \\
& \left.\quad-\sqrt{-1}\left[\alpha\left(x^{\prime}\right) \tilde{\boldsymbol{q}}_{0}\left(x^{\prime}, \xi^{\prime}, y, \eta\right)+\sum_{j=1}^{2} \partial_{x_{j}}\left(\alpha\left(x^{\prime}\right) \cdot \partial_{\xi_{j}} \tilde{\boldsymbol{p}}_{1}\left(x^{\prime}, \xi^{\prime}, y, \eta\right)\right)\right]\right) \\
& \quad+\text { terms of order } \leq-1 .
\end{aligned}
$$

However, by virtue of Lemma 5.4 it follows that

$$
\partial_{x_{j}} \alpha\left(x^{\prime}\right)=0 \quad \text { on } M=\left\{x^{\prime} \in \partial \Omega: \alpha\left(x^{\prime}\right)=0\right\} .
$$


Thus we can easily verify that the pseudo-differential operator $\tilde{\boldsymbol{T}}^{*}$ satisfies conditions (2.7a) and (2.7b) of a matrix-valued version of Theorem 2.12 with $\mu:=0$, $\rho:=1$ and $\delta:=1 / 2$. This implies that estimate (7.5) remains valid for the adjoint operator $\tilde{\mathscr{T}}_{\alpha}^{*}$ of $\tilde{\mathscr{T}}_{\alpha}$ :

$$
|\tilde{\boldsymbol{\psi}}|_{-s+1 / p, p^{\prime}} \leq \tilde{C}_{\tau}\left(\left|\tilde{\boldsymbol{T}}_{\alpha}^{*} \tilde{\boldsymbol{\psi}}\right|_{-s+1 / p, p^{\prime}}+|\tilde{\boldsymbol{\psi}}|_{\tau, p^{\prime}}\right), \quad \tilde{\boldsymbol{\psi}} \in D\left(\tilde{\mathscr{T}}_{\alpha}^{*}\right),
$$

where $\tau<-s+1 / p$ and $p^{\prime}=p /(p-1)$ is the exponent conjugate to $p$. Hence we have, by the closed range theorem (cf. [Yo, Chapter VII, Section 5]) and Lemma 7.5,

$$
\operatorname{codim} R\left(\tilde{\mathscr{T}}_{\alpha}\right)=\operatorname{dim} N\left(\tilde{\mathscr{T}}_{\alpha}^{*}\right)<\infty,
$$

since the injection $\boldsymbol{B}^{-s+1 / p, p^{\prime}}\left(\partial \Omega \times S, \mathbf{R}^{3}\right) \rightarrow \boldsymbol{B}^{\tau, p^{\prime}}\left(\partial \Omega \times S, \mathbf{R}^{3}\right)$ is compact for $\tau<-s+1 / p$.

Therefore, the desired assertion (7.4) follows from assertions (7.6) and (7.7).

Step 3-b: By assertion (7.4), we can apply Proposition 7.4 to obtain that the operator $\mathscr{T}_{\alpha}\left(\ell^{2}\right): \boldsymbol{B}^{s-1 / p, p}\left(\partial \Omega, \mathbf{R}^{3}\right) \rightarrow \boldsymbol{B}^{s-1 / p, p}\left(\partial \Omega, \mathbf{R}^{3}\right)$ is bijective if $\ell \in \mathbf{Z} \backslash K$ for some finite subset $K$ of $\mathbf{Z}$. In particular, we have the formula

$$
\text { ind } \mathscr{T}_{\alpha}\left(\lambda_{0}\right)=0 \text { if } \lambda_{0}=\ell^{2}, \ell \in \mathbf{Z} \backslash K \text {. }
$$

However, it is easy to see that the symbol $\boldsymbol{t}\left(x^{\prime}, \xi^{\prime} ; \lambda\right)$ of the operator

$$
\boldsymbol{T}_{\alpha}(\lambda)=\alpha\left(x^{\prime}\right) \boldsymbol{\Pi}(\lambda)+\left(1-\alpha\left(x^{\prime}\right)\right) \boldsymbol{I}, \quad \lambda \geq 0,
$$

has the following asymptotic expansion:

$$
\begin{aligned}
\boldsymbol{t}\left(x^{\prime}, \xi^{\prime} ; \lambda\right)= & \alpha\left(x^{\prime}\right)\left[\boldsymbol{p}_{1}\left(x^{\prime}, \xi^{\prime}\right)+\sqrt{-1} \boldsymbol{q}_{1}\left(x^{\prime}, \xi^{\prime}\right)\right] \\
& +\left[\left(1-\alpha\left(x^{\prime}\right)+\alpha\left(x^{\prime}\right) \boldsymbol{p}_{0}\left(x^{\prime}, \xi\right)\right)+\sqrt{-1} \alpha\left(x^{\prime}\right) \boldsymbol{q}_{0}\left(x^{\prime}, \xi^{\prime}\right)\right] \\
& + \text { terms of order } \leq-1 \text { depending on } \lambda .
\end{aligned}
$$

Thus, by taking $\lambda:=0$ and $\lambda:=\lambda_{0}$ in formula (7.9) we can find a $3 \times 3$ matrixvalued, classical pseudo-differential operator $\boldsymbol{K}\left(0, \lambda_{0}\right)$ of order -1 on the boundary $\partial \Omega$ such that

$$
\boldsymbol{T}_{\alpha}=\boldsymbol{T}_{\alpha}\left(\lambda_{0}\right)+\boldsymbol{K}\left(0, \lambda_{0}\right) .
$$

Furthermore, the Rellich-Kondrachov theorem asserts that the operator

$$
\boldsymbol{K}\left(0, \lambda_{0}\right): \boldsymbol{B}^{s-1 / p, p}\left(\partial \Omega, \mathbf{R}^{3}\right) \rightarrow \boldsymbol{B}^{s-1 / p, p}\left(\partial \Omega, \mathbf{R}^{3}\right)
$$

is compact. Hence we have the formula 


$$
\text { ind } \mathscr{T}_{\alpha}=\text { ind } \mathscr{T}_{\alpha}\left(\lambda_{0}\right) \text {. }
$$

Therefore, Proposition 7.2 follows by combining formulas (7.10) and (7.8). Now the proof of Proposition 7.2 is complete.

Theorem 7.1 is proved, apart from the proof of Proposition 7.4. The proof of Proposition 7.4 is given in the next Section 7.2, due to its length.

\subsection{Proof of Proposition 7.4}

The proof of Proposition 7.4 is divided into three steps.

Step 1: First, we study the null spaces $N\left(\tilde{\mathscr{T}}_{\alpha}\right)$ and $N\left(\mathscr{T}_{\alpha}\left(\lambda^{\prime}\right)\right)$ when $\lambda^{\prime}=\ell^{2}$ with $\ell \in \mathbf{Z}$ :

$$
\begin{aligned}
& N\left(\tilde{\mathscr{T}}_{\alpha}\right)=\left\{\tilde{\boldsymbol{\varphi}} \in \boldsymbol{B}^{s-1 / p, p}\left(\partial \boldsymbol{\Omega} \times S, \mathbf{R}^{3}\right): \tilde{\boldsymbol{T}}_{\alpha} \tilde{\boldsymbol{\varphi}}=0\right\}, \\
& N\left(\mathscr{T}_{\alpha}\left(\lambda^{\prime}\right)\right)=\left\{\boldsymbol{\varphi} \in \boldsymbol{B}^{s-1 / p, p}\left(\partial \boldsymbol{\Omega}, \mathbf{R}^{3}\right): \boldsymbol{T}_{\alpha}\left(\lambda^{\prime}\right) \boldsymbol{\varphi}=\mathbf{0}\right\} .
\end{aligned}
$$

Since the pseudo-differential operators $\tilde{\boldsymbol{T}}_{\alpha}$ and $\boldsymbol{T}_{\alpha}\left(\lambda^{\prime}\right)$ are both hypoelliptic, it follows that

$$
\begin{aligned}
& N\left(\tilde{\mathscr{T}}_{\alpha}\right)=\left\{\tilde{\boldsymbol{\varphi}} \in \boldsymbol{C}^{\infty}\left(\partial \Omega \times S, \mathbf{R}^{3}\right): \tilde{\boldsymbol{T}}_{\alpha} \tilde{\boldsymbol{\varphi}}=\mathbf{0}\right\}, \\
& N\left(\mathscr{T}_{\alpha}\left(\lambda^{\prime}\right)\right)=\left\{\boldsymbol{\varphi} \in \boldsymbol{C}^{\infty}\left(\partial \boldsymbol{\Omega}, \mathbf{R}^{3}\right): \boldsymbol{T}_{\alpha}\left(\lambda^{\prime}\right) \boldsymbol{\varphi}=\mathbf{0}\right\} .
\end{aligned}
$$

Therefore, we can apply [Ta1, Proposition 8.4.6] to obtain the following most important relationship between the null spaces $N\left(\tilde{\mathscr{T}}_{\alpha}\right)$ and $N\left(\mathscr{T}_{\alpha}\left(\lambda^{\prime}\right)\right)$ when $\lambda^{\prime}=\ell^{2}$ with $\ell \in \mathbf{Z}$ :

Lemma 7.6. The following two conditions (1) and (2) are equivalent:

(1) $\operatorname{dim} N\left(\tilde{\mathscr{T}}_{\alpha}\right)<\infty$.

(2) There exists a finite subset I of $\mathbf{Z}$ such that

$$
\begin{cases}\operatorname{dim} N\left(\mathscr{T}_{\alpha}\left(\ell^{2}\right)\right)<\infty & \text { if } \ell \in I, \\ \operatorname{dim} N\left(\mathscr{T}_{\alpha}\left(\ell^{2}\right)\right)=0 & \text { if } \ell \notin I .\end{cases}
$$

Moreover, in this case we have the formulas

$$
\begin{aligned}
& N\left(\tilde{\mathscr{T}}_{\alpha}\right)=\bigoplus_{\ell \in I} N\left(\mathscr{T}_{\alpha}\left(\ell^{2}\right)\right) \otimes \ell^{i \ell y}, \\
& \operatorname{dim} N\left(\tilde{\mathscr{T}}_{\alpha}\right)=\sum_{\ell \in I} \operatorname{dim} N\left(\mathscr{T}_{\alpha}\left(\ell^{2}\right)\right) .
\end{aligned}
$$


Step 2: Secondly, we study the ranges $R\left(\tilde{\mathscr{T}}_{\alpha}\right)$ and $R\left(\mathscr{T}_{\alpha}\left(\lambda^{\prime}\right)\right)$ when $\lambda^{\prime}=\ell^{2}$ with $\ell \in \mathbf{Z}$. To do this, we consider the adjoint operators $\tilde{\mathscr{T}}_{\alpha}^{*}$ and $\mathscr{T}_{\alpha}\left(\lambda^{\prime}\right)^{*}$ of $\tilde{\mathscr{T}}_{\alpha}$ and $\mathscr{T}_{\alpha}\left(\lambda^{\prime}\right)$, respectively.

The next lemma allows us to give a characterization of the adjoint operators $\tilde{\mathscr{T}}_{\alpha}^{*}$ and $\mathscr{T}_{\alpha}\left(\lambda^{\prime}\right)^{*}$ in terms of pseudo-differential operators (cf. [Ta1, Lemma 8.4.8]):

Lemma 7.7. Let $M$ be an n-dimensional, compact smooth manifold without boundary. If $T$ is a classical pseudo-differential operator of order $m$ on $M$, we define a densely defined, closed linear operator

$$
\mathscr{T}: B^{s, p}(M) \rightarrow B^{s-m+1, p}(M) \quad(s \in \mathbf{R})
$$

as follows.

(a) The domain $D(\mathscr{T})$ of $\mathscr{T}$ is the space

$$
D(\mathscr{T})=\left\{\varphi \in H^{s, p}(M): T \varphi \in H^{s-m+1, p}(M)\right\} .
$$

(b) $\mathscr{T} \varphi=T \varphi, \varphi \in D(\mathscr{T})$.

Then the adjoint operator $\mathscr{T}^{*}$ of $\mathscr{T}$ is characterized as follows:

(c) The domain $D\left(\mathscr{T}^{*}\right)$ of $\mathscr{T}^{*}$ is contained in the space

$$
\left\{\psi \in B^{-s+m-1, p^{\prime}}(M): T^{*} \psi \in B^{-s, p^{\prime}}(M)\right\},
$$

where $p^{\prime}=p /(p-1)$ and $T^{*} \in L_{\mathrm{cl}}^{m}(M)$ is the adjoint of $T$.

(d) $\mathscr{T}^{*} \psi=T^{*} \psi, \psi \in D\left(\mathscr{T}^{*}\right)$.

It should be noticed that the pseudo-differential operators $\boldsymbol{T}(\lambda)^{*}$ and $\tilde{\boldsymbol{T}}^{*}$ also satisfy conditions (2.7a) and (2.7b) of a matrix-valued version of Theorem 2.12 with $\mu:=0, \rho:=1$ and $\delta:=1 / 2$; hence they are hypoelliptic.

Therefore, by applying Lemma 7.7 to the operators $\tilde{\boldsymbol{T}}$ and $\boldsymbol{T}\left(\lambda^{\prime}\right)$ we obtain the following:

Lemma 7.8. The null spaces $N\left(\tilde{\mathscr{T}}_{\alpha}^{*}\right)$ and $N\left(\mathscr{T}_{\alpha}\left(\lambda^{\prime}\right)^{*}\right)$ are characterized respectively as follows:

$$
\begin{aligned}
& N\left(\tilde{\mathscr{T}}_{\alpha}^{*}\right)=\left\{\tilde{\boldsymbol{\psi}} \in \boldsymbol{C}^{\infty}\left(\partial \boldsymbol{\Omega} \times S, \mathbf{R}^{3}\right): \tilde{\boldsymbol{T}}_{\alpha}^{*} \tilde{\boldsymbol{\psi}}=\mathbf{0}\right\} . \\
& N\left(\mathscr{T}_{\alpha}\left(\lambda^{\prime}\right)^{*}\right)=\left\{\boldsymbol{\psi} \in \boldsymbol{C}^{\infty}\left(\partial \Omega, \mathbf{R}^{3}\right): \boldsymbol{T}_{\alpha}\left(\lambda^{\prime}\right)^{*} \boldsymbol{\psi}=\mathbf{0}\right\} .
\end{aligned}
$$

By Lemma 7.8, we find that Lemma 7.6 remains valid for the adjoint operators $\tilde{\mathscr{T}}_{\alpha}^{*}$ and $\mathscr{T}_{\alpha}\left(\lambda^{\prime}\right)^{*}$ (cf. [Ta1, Lemma 8.4.10]): 
Lemma 7.9. The following two conditions (1) and (2) are equivalent:

(1) $\operatorname{dim} N\left(\tilde{\mathscr{T}}_{\alpha}^{*}\right)<\infty$.

(2) There exists a finite subset $J$ of $\mathbf{Z}$ such that

$$
\begin{cases}\operatorname{dim} N\left(\mathscr{T}_{\alpha}\left(\ell^{2}\right)^{*}\right)<\infty & \text { if } \ell \in J, \\ \operatorname{dim} N\left(\mathscr{T}_{\alpha}\left(\ell^{2}\right)^{*}\right)=0 & \text { if } \ell \notin J .\end{cases}
$$

Moreover, in this case we have the formula

$$
\operatorname{dim} N\left(\tilde{\mathscr{T}}_{\alpha}^{*}\right)=\sum_{\ell \in J} \operatorname{dim} N\left(\mathscr{T}_{\alpha}\left(\ell^{2}\right)^{*}\right)
$$

Hence, by combining Lemma 7.9 and the closed range theorem we obtain the most important relationship between codim $R\left(\tilde{\mathscr{T}}_{\alpha}\right)$ and $\operatorname{codim} R\left(\mathscr{T}_{\alpha}\left(\lambda^{\prime}\right)\right)$ when $\lambda^{\prime}=\ell^{2}, \ell \in \mathbf{Z}$ (cf. [Ta1, Proposition 8.4.11]):

LEMma 7.10. The following two conditions (1) and (2) are equivalent:

(1) $\operatorname{codim} R\left(\tilde{\mathscr{T}}_{\alpha}\right)<\infty$.

(2) There exists a finite subset $J$ of $\mathbf{Z}$ such that

$$
\begin{cases}\operatorname{codim} R\left(\mathscr{T}_{\alpha}\left(\ell^{2}\right)\right)<\infty & \text { if } \ell \in J, \\ \operatorname{codim} N\left(\mathscr{T}_{\alpha}\left(\ell^{2}\right)\right)=0 & \text { if } \ell \notin J .\end{cases}
$$

Moreover, in this case we have the formula

$$
\operatorname{codim} R\left(\tilde{\mathscr{T}}_{\alpha}\right)=\sum_{\ell \in J} \operatorname{codim} R\left(\mathscr{T}_{\alpha}\left(\ell^{2}\right)\right) .
$$

Step 3: Proposition 7.4 is an immediate consequence of Lemmas 7.6 and 7.10, with $K:=I \cup J$.

Now the proof of Proposition 7.4 and hence that of Theorem 7.1 is complete.

\section{Proof of Theorems}

This chapter is devoted to the proof of Main Theorem, Theorem 1.1 and Theorem 1.2. After reviewing some differential calculus in Banach spaces in Subsection 8.1.1, we prove Main Theorem in Section 8.1. A basic result that relates linearized and nonlinear theories is the inverse mapping theorem (Theorem 8.1). In the proof of Theorems 1.1 and 1.2 , we calculate explicitly the first 
elasticity tensor $\AA$, and verify that either condition (B) or condition (C) implies condition (S) in Sections 8.2 and 8.3.

\subsection{Proof of Main Theorem}

By the existence and uniqueness theorem for problem (1.4) (Theorem 3.1), our Main Theorem follows from an application of the inverse mapping theorem (Theorem 8.1).

8.1.1 The Inverse Mapping Theorem. Let $\mathscr{X}$ and $\mathscr{Y}$ be Banach spaces, $\mathscr{U}$ an open set in $\mathscr{X}$ and $f: \mathscr{U} \rightarrow \mathscr{Y}$ a map. We say that the map $f$ is differentiable at a point $x$ of $\mathscr{U}$ if there exist a continuous linear operator $A: \mathscr{X} \rightarrow \mathscr{Y}$ and a map $\psi$ defined for all sufficiently small $h$ in $\mathscr{X}$, with values in $\mathscr{Y}$, such that

$$
\left\{\begin{array}{l}
f(x+h)=f(x)+A h+\|h\| \psi(h), \\
\lim _{h \rightarrow 0} \psi(h)=0 .
\end{array}\right.
$$

It should be emphasized that the continuous linear operator $A$ is uniquely determined by $f$ and $x$. The operator $A$ is called the Fréchet derivative of $f$ at $x$, and is denoted by $f^{\prime}(x)$ or $D f(x)$. A map $f$ is said to be differentiable on $\mathscr{U}$ if it is differentiable at every point of $\mathscr{U}$. In this case, the derivative $f^{\prime}$ is a map of $\mathscr{U}$ into the Banach space $\mathscr{B}(\mathscr{X}, \mathscr{Y})$ of continuous linear operators:

$$
\begin{aligned}
D f=f^{\prime}: \mathscr{U} & \rightarrow \mathscr{B}(\mathscr{X}, \mathscr{Y}) \\
x & \mapsto f^{\prime}(x) .
\end{aligned}
$$

If $f^{\prime}$ is continuous from $\mathscr{U}$ into $\mathscr{B}(\mathscr{X}, \mathscr{Y})$, we say that $f$ is of class $C^{1}$.

We can define inductively the derivatives $D^{k} f$ for general $k \geq 2$. A map $f$ is said to be of class $C^{r}(r \geq 2)$ if all derivatives $D^{k} f$ exist and are continuous for $1 \leq k \leq r$.

The next inverse mapping theorem provides a criterion for a map to be a local $C^{r}$-diffeomorphism in terms of its derivative (cf. [MH, Chapter 4, Theorem 1.2]):

THEOREM 8.1 (The inverse mapping theorem). Let $\mathscr{X}$ and $\mathscr{Y}$ be Banach spaces, and let $f$ be a $C^{r}$-map $(r \geq 1)$ of an open subset $\mathscr{U}$ of $\mathscr{X}$ into $\mathscr{Y}$. Assume that the Fréchet derivative $f^{\prime}\left(x_{0}\right): \mathscr{X} \rightarrow \mathscr{Y}$ is an algebraic and topological isomorphism at a point $x_{0}$ of $\mathscr{U}$. Then the map $f$ is a $C^{r}$-diffeomorphism of some neighborhood of $x_{0}$ onto some neighborhood of $f\left(x_{0}\right)$.

8.1.2 Proof of Main Theorem. We recall that the linearization of problem (1.1) is nothing but problem (1.3) or problem (1.4) with $\mathbf{a}(x):=\stackrel{\AA}{\mathbf{A}}(X)$. However, Theorem 3.1 asserts that: 
The Fréchet derivative $F^{\prime}\left(\stackrel{\circ}{)}\right.$ of the map $F$ at $\stackrel{\phi}{\boldsymbol{\phi}}=\boldsymbol{I}_{\Omega}$ is an algebraic and topological isomorphism of $\boldsymbol{H}^{s, p}\left(\Omega, \mathbf{R}^{3}\right)$ onto $\boldsymbol{H}^{s-2, p}\left(\Omega, \mathbf{R}^{3}\right) \times \boldsymbol{B}_{(\alpha)}^{s-1-1 / p, p}\left(\partial \Omega, \mathbf{R}^{3}\right)$.

Therefore, Main Theorem follows immediately from an application of the inverse mapping theorem (Theorem 8.1).

\subsection{Proof of Theorem 1.1}

The stored energy function $W(X, \boldsymbol{F})$ for the Hencky-Nadai elasto-plastic material has the form

$$
W(X, \boldsymbol{F})=\frac{3}{4} \int_{0}^{\Gamma(\boldsymbol{F})} g(\xi) d \xi+\frac{K}{2}\left(\sum_{k=1}^{3} F_{k k}-3\right)^{2},
$$

where $g \in C^{\infty}([0, \infty), \mathbf{R})$, the constant $K$ is the modulus of compression and

$$
\Gamma(\boldsymbol{F})=\frac{4}{3} \sum_{i, j=1}^{3}\left(\frac{1}{2}\left(F_{i j}+F_{j i}\right)-\frac{1}{3}\left(\sum_{k=1}^{3} F_{k k} F_{k k}\right) \delta_{i j}\right)^{2} .
$$

We have only to verify condition (S). First, it follows (cf. [MH, Chapter 3, Proposition 4.4]) that the first Piola-Kirchhoff stress tensor $\boldsymbol{P}(X)=\left(P_{i j}(X)\right)$ is given by the formula

$$
\begin{aligned}
P_{i j}(X)= & \hat{P}_{i j}(X, \boldsymbol{F}(X)) \\
= & \frac{\partial W}{\partial F_{i j}}(X, \boldsymbol{F}(X)) \\
= & \left(K-\frac{2}{3} g(\Gamma(\boldsymbol{F}(X)))\right)\left(\sum_{k=1}^{3} F_{k k}(X)-3\right) \delta_{i j} \\
& +g(\Gamma(\boldsymbol{F}(X)))\left(F_{i j}(X)+F_{j i}(X)-2 \delta_{i j}\right),
\end{aligned}
$$

and that the first elasticity tensor $\mathbf{A}(X)=\left(\mathrm{A}_{i j / m}(X)\right)$ is given by the formula

$$
\begin{aligned}
\mathrm{A}_{i j \ell m}(X)= & \frac{\partial \hat{\boldsymbol{P}}_{i j}}{\partial F_{\ell m}}(X, \boldsymbol{F}(X)) \\
= & g(\Gamma(\boldsymbol{F}(X)))\left(\delta_{i \ell} \delta_{j m}+\delta_{i m} \delta_{j \ell}\right)+\left(K-\frac{2}{3} g(\Gamma(\boldsymbol{F}(X)))\right) \delta_{i j} \delta_{\ell m} \\
& +\frac{16}{3} g^{\prime}(\Gamma(\boldsymbol{F}(X))) \bar{\varepsilon}_{i j}(\boldsymbol{F}(X)) \bar{\varepsilon}_{\ell m}(\boldsymbol{F}(X)),
\end{aligned}
$$

where 


$$
\begin{aligned}
& \bar{\varepsilon}_{i j}(\boldsymbol{F}(X))=\frac{1}{2}\left(F_{i j}(X)+F_{j i}(X)\right)-\frac{1}{3}\left(\sum_{k=1}^{3} F_{k k}(X) F_{k k}(X)\right) \delta_{i j}, \\
& \boldsymbol{F}(X)=\left(F_{i j}(X)\right)=\left(\frac{\partial \phi_{i}}{\partial X_{j}}\right) .
\end{aligned}
$$

Thus we find that the elasticity tensor $\stackrel{\circ}{\mathbf{A}}(X)=\left(\AA_{i j \ell m}(X)\right)$ evaluated at $\stackrel{\circ}{\boldsymbol{\phi}}=\boldsymbol{I}_{\Omega}$ is equal to the following:

$$
\stackrel{\circ}{i j \ell m}_{i}(X)=g(0)\left(\delta_{i \ell} \delta_{j m}+\delta_{i m} \delta_{j \ell}\right)+\left(K-\frac{2}{3} g(0)\right) \delta_{i j} \delta_{\ell m} .
$$

However, it is easy to see (cf. [MH, Chapter 4, Proposition 3.13]) that the elasticity tensor $\stackrel{\AA}{\mathbf{A}}(X)$ is uniformly pointwise stable if and only if $g(0)>0$ and $K>0$.

Therefore, we have proved that condition (B) implies condition (S).

The proof of Theorem 1.1 is complete.

\subsection{Proof of Theorem 1.2}

The stored energy function $W(X, \boldsymbol{F})$ for the Saint Venant-Kirchhoff isotropic material has the form

$$
W(X, \boldsymbol{F})=\frac{\lambda(X)}{8}\left(\sum_{k=1}^{3} C_{k k}(\boldsymbol{F})-3\right)^{2}+\frac{\mu(X)}{4} \sum_{i, j=1}^{3}\left(C_{i j}(\boldsymbol{F})-\delta_{i j}\right)^{2},
$$

where $\lambda(X), \mu(X)$ are smooth Lamé functions, and the two-tensor

$$
\boldsymbol{C}=\left(C_{i j}(\boldsymbol{F})\right)=\left(\sum_{k=1}^{3} F_{k i} F_{k j}\right)
$$

is the (right) Cauchy-Green strain tensor.

We have only to verify condition (S). First, it follows (cf. [MH, Chapter 3, Proposition 4.4]) that the second Piola-Kirchhoff stress tensor $\boldsymbol{S}(X)=$ $\left(\hat{S}_{i j}(X, C(X))\right)$ is given by the formula

$$
\begin{aligned}
\hat{S}_{i j}(X, \boldsymbol{C}(X)) & =2 \frac{\partial W}{\partial C_{i j}}(X, \boldsymbol{F}(X)) \\
& =\left[\frac{\lambda(X)}{2}\left(\sum_{k=1}^{3} C_{k k}(X)-3\right)-\mu(X)\right] \delta_{i j}+\mu(X) C_{i j}(X),
\end{aligned}
$$


and that the second elasticity tensor $\mathrm{C}(X)=\left(\mathrm{C}_{i j \ell m}(X)\right)$ is given by the formula

$$
\mathrm{C}_{i j \ell m}(X)=\frac{\partial \hat{S}_{i j}}{\partial C_{\ell m}}(X, C(X))=\frac{\lambda(X)}{2} \delta_{i j} \delta_{\ell m}+\frac{\mu(X)}{2}\left(\delta_{i \ell} \delta_{j m}+\delta_{i m} \delta_{j \ell}\right) .
$$

Then we find (cf. [MH, Chapter 3, Proposition 4.5]) that the first elasticity tensor $\mathbf{A}(X)=\left(\mathrm{A}_{i j \ell m}(X)\right)$ is given by the following formula:

$$
\begin{aligned}
\mathrm{A}_{i j \ell m}(X)= & 2 \sum_{a, b=1}^{3} \mathrm{C}_{a j b m}(X) F_{i a}(X) F_{\ell b}(X)+\hat{S}_{j m}(X, \boldsymbol{C}(X)) \delta_{i \ell} \\
= & \sum_{a, b=1}^{3}\left(\lambda(X) \delta_{a j} \delta_{b m}+\mu(X)\left(\delta_{a b} \delta_{j m}+\delta_{a m} \delta_{b j}\right)\right) F_{i a}(X) F_{\ell b}(X) \\
& +\left(\left[\frac{\lambda(X)}{2}\left(\sum_{k=1}^{3} C_{k k}(\boldsymbol{F}(X))-3\right)-\mu(X)\right] \delta_{j m}+\mu(X) C_{j m}(\boldsymbol{F}(X))\right) \delta_{i \ell} .
\end{aligned}
$$

Thus it follows that the elasticity tensor $\stackrel{\circ}{\mathbf{A}}(X)=\left(\AA_{i j \ell m}(X)\right)$ evaluated at $\stackrel{\circ}{\boldsymbol{\phi}}=\boldsymbol{I}_{\Omega}$ is equal to the following:

$$
\stackrel{\circ}{\mathrm{A}}_{i j \ell m}(X)=\mu(X)\left(\delta_{i \ell} \delta_{j m}+\delta_{i m} \delta_{j \ell}\right)+\lambda(X) \delta_{i j} \delta_{\ell m} .
$$

However, it is easy to see (cf. [MH, Chapter 4, Proposition 3.13]) that if condition $(\mathrm{C})$ is satisfied, then the elasticity tensor $\mathbf{A}(X)$ is uniformly pointwise stable. This proves that condition (C) implies condition (S).

The proof of Theorem 1.2 is complete.

\section{Summary and Discussion}

We have studied boundary value problems of nonlinear elastostatics in the case where solutions of the linearized problem correspond faithfully to those of the nonlinear problem, that is, in the case where there is no bifurcation. We have proved that if the linearized problem has unique solutions, then so does the nonlinear one, nearby (Main Theorem). This is done by using the $L^{p}$ theory of pseudo-differential operators and the inverse mapping theorem. Our boundary condition is a "regularization" of the genuine mixed displacement-traction boundary condition; more precisely, it is a smooth linear combination of displacement and traction boundary conditions, but is not equal to the pure traction boundary condition. Moreover, it should be emphasized that our problem becomes a degenerate elliptic boundary value problem from an analytical point of view. The crucial point is how to find a function space associated with the 
degenerate boundary condition in which the linearized problem has unique solutions. Main Theorem could be applied to the Saint Venant-Kirchhoff elastic material and the Hencky-Nadai elasto-plastic material (Theorem 1.1 and Theorem 1.2). Some previous results with pure displacement boundary condition are due to Ciarlet [Ci], Dinca [Di], Marsden-Hughes [MH] and Valent [Va]. The results here have extended and improved substantially those results in a unified theory. Our approach is distinguished by the extensive use of the ideas and techniques characteristic of the recent developments in the theory of partial differential equations ([Ta4]).

Finally, we give two important open problems concerning the boundary value problems of nonlinear elastostatics (see Figure 9.1):

(1) The first problem is to generalize main results to the case where the domain $\Omega$ has corner singularities.

(2) The second problem is to study the case where the function $\alpha(x)$ is the characteristic function of a subset of the boundary $\partial \Omega$.

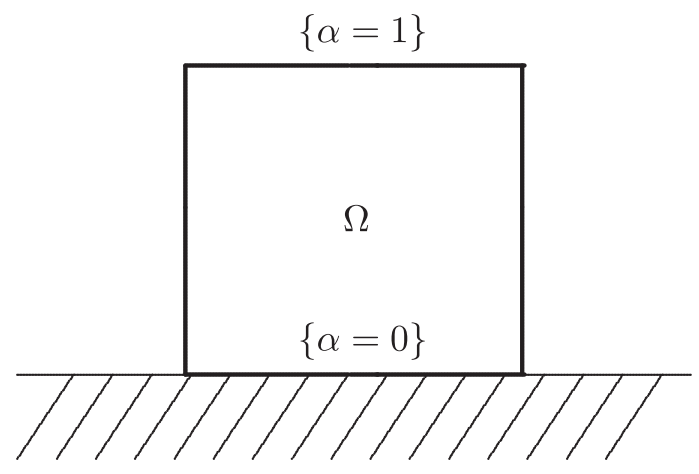

Figure 9.1

It should be emphasized that Ito [It2] obtained some important results in the framework of Sobolev spaces of $L^{2}$ type, by using Melin's inequality [Me].

We leave these open problems for future study.

\section{Acknowledgements}

The author is grateful to the referee for many valuable suggestions which improved the presentation of this paper. This research was partially supported by Grant-in-Aid for General Scientific Research (No. 16340031), Ministry of Education, Culture, Sports, Science and Technology, Japan. 


\section{References}

[AF] R. A. Adams and J. J. F. Fournier, Sobolev spaces, second edition, Academic Press, Amsterdam Heidelberg New York Oxford, 2003.

[Ag] S. Agmon, Lectures on elliptic boundary value problems, Van Nostrand, Princeton, 1965.

[BL] J. Bergh and J. Löfström, Interpolation spaces, an introduction, Springer-Verlag, Berlin New York Heidelberg, 1976.

[Bo] G. Bourdaud, $L^{p}$-estimates for certain non-regular pseudo-differential operators, Comm. in Partial Differential Equations 7 (1982), 1023-1033.

[CP] J. Chazarain et A. Piriou, Introduction à la théorie des équations aux dérivées partielles linéaires, Gauthier-Villars, Paris, 1981.

[Ci] P. G. Ciarlet, Mathematical elasticity, Vol. I; Studies in mathematics and its applications, No. 20, North-Holland, Amsterdam New York Oxford Tokyo, 1988.

[Di] G. Dinca, Sur la monotonie d'après Minty-Browder de l'opérateur de la théorie de plasticité, C. R. Acad. Sci. Paris 269 (1969), 535-538.

[DL] G. Duvaut et J.-L. Lions, Les inéquations en mécanique et en physique, Dunod, Paris, 1972.

[Fr] A. Friedman, Partial differential equations, Holt, Rinehart and Winston, New York, 1969.

[Hol] L. Hörmander, Pseudodifferential operators and non-elliptic boundary problems, Ann. of Math. 83 (1966), 129-209.

[Ho2] L. Hörmander, Pseudo-differential operators and hypoelliptic equations, Proc. Sym. Pure Math. Vol. X (Singular integrals), Amer. Math. Soc., Providence, Rhode Island, 1967, pp. $138-183$.

[Ho3] L. Hörmander, The analysis of linear partial differential operators, Vol. III, Springer-Verlag, New York Berlin Heidelberg Tokyo, 1983.

[It1] H. Ito, On certain mixed-type boundary-value problems of elastostatics, Tsukuba J. Math. 14 (1990), 133-153.

[It2] H. Ito, On a mixed problem of linear elastodynamics with a time-dependent discontinuous boundary condition, Osaka J. Math. 27 (1990), 667-707.

[Ku] H. Kumano-go, Pseudodifferential operators, MIT Press, Cambridge, Massachusetts, 1981.

[LM] J.-L. Lions et E. Magenes, Problèmes aux limites non-homogènes et applications, Vols. 1, 2, Dunod, Paris, 1968; English translation, Springer-Verlag, Berlin New York Heidelberg, 1972.

$[\mathrm{MH}]$ J. E. Marsden and T. J. R. Hughes, Mathematical foundations of elasticity, Prentice-Hall, Inc., Englewood Cliffs, New Jersey, 1983.

[Me] A. Melin, Lower bounds for pseudo-differential operators, Ark. för Mat. 9 (1971), 117-140.

[Se] R. T. Seeley, Singular integrals and boundary value problems, Amer. J. Math. 88 (1966), 781-809.

[St] E. M. Stein, The characterization of functions arising as potentials II, Bull. Amer. Math. Soc. 68 (1962), 577-582.

[Ta1] K. Taira, Diffusion processes and partial differential equations, Academic Press, San Diego New York London Tokyo, 1988.

[Ta2] K. Taira, Analytic semigroups and semilinear initial boundary value problems, London Mathematical Society Lecture Note Series, No. 223, Cambridge University Press, London New York, 1995.

[Ta3] K. Taira, On boundary value problems of nonlinear elastostatics, Osaka J. Math. 33 (1996), $555-585$.

[Ta4] K. Taira, Semigroups, boundary value problems and Markov processes, Springer-Verlag, Berlin Heidelberg New York, 2004.

[Ty] M. Taylor, Pseudodifferential operators, Princeton University Press, Princeton, 1981.

[Tr] H. Triebel, Interpolation theory, function spaces, differential operators, North-Holland, Amsterdam, 1978. 
[Va] T. Valent, Boundary value problems of finite elasticity; Springer tracts in natural philosophy, No. 31, Springer-Verlag, New York Berlin Heidelberg Tokyo, 1988.

[Yo] K. Yosida, Functional analysis, sixth edition, Springer-Verlag, Berlin Heidelberg New York, 1980.

\author{
Institute of Mathematics \\ University of Tsukuba \\ Tsukuba 305-8571, Japan \\ E-mail address: taira@math.tsukuba.ac.jp
}

